\title{
Conexidade Fuzzy Relativa em Grafos Dirigidos e sua Aplicação em um Método Híbrido para Segmentação Interativa de Imagens
}

\author{
Hans Harley Ccacyahuillca Bejar
}

\author{
DISSERTAÇÃO APRESENTADA \\ $\mathrm{AO}$ \\ Instituto De MatemáticA E EstatísticA \\ DA \\ Universidade DE SÃo PAUlo \\ PARA \\ OBTENÇÃO DO TÍTULO \\ $\mathrm{DE}$ \\ Mestre EM CiÊnCIAS
}

Programa: Ciência da Computação

Orientador: Prof. Dr. Paulo André Vechiatto de Miranda.

Durante o desenvolvimento deste trabalho o autor recebeu auxílio financeiro da CAPES

São Paulo, abril de 2016 


\section{Conexidade Fuzzy Relativa em Grafos Dirigidos e sua Aplicação em um Método Híbrido para Segmentação Interativa de Imagens}

Esta versão da dissertação contém as correções e alterações sugeridas pela Comissão Julgadora durante a defesa da versão original do trabalho, realizada em 27/04/2016. Uma cópia da versão original está disponível no Instituto de Matemática e Estatística da Universidade de São Paulo.

Comissão Julgadora:

- Prof. Dr. Paulo André Vechiatto de Miranda (orientador) - IME-USP

- Prof. Dr. Ronaldo Fumio Hashimoto - IME-USP

- Prof. Dr. Alexandre Noma - UFABC 


\section{Agradecimentos}

Gostaria de agredecer a todos que fizeram possível concretiçar esse objetivo de forma direta ou indireta:

Ao meu orientador, o Prof. Dr. Paulo André Vechiatto de Miranda, pela orientação, paciência e principalmente pela dedicação na pesquisa científica.

Aos professores membros da banca de qualificação e defesa à Dra. Nina Sumiko Tomita Hirata, ao Dr. Ronaldo Hashimoto e ao Dr. Alexandre Noma por as observações e conselhos.

Ao IME-USP pela oportunidade e experiência professional fornecida e à CAPES pelo apoio financiero.

Ao Dr. J. K. Udupa (MIPG-UPENN) pelas imagens médicas e ao Prof. Fernando Cendes da Faculdade de Ciências Médicas da UNICAMP pelas imagens do cérebro.

Ao grupo eScience-IME-USP: Jihan, Silvia, Jorge, Leissi, Anderson, Edu, Leandro, Frank, Waldir, Grover, Rocio, Urpy, Nancy, Miguel, Mariela, Lucy, Susana, Amanda, Lulu, Evelyn, Maciel, Fernando, Paulo, Caio, Sabrina, Juan, e também aos meus amigos do IME: Fabiano, Julio, Raul, Hugo, Marcos, Marisol e Erika, pessoas maravilhosas que conheci ao longo do mestrado.

Ao grupo ACM-Chapter-Cusco INFO-UNSAAC pela organização dos eventos no final do ano, que permitem compartilhar conhecimentos entre estudante de Cusco-Perú que estudam em diferentes partes do mundo, particularmente agradeço ao Prof. Msc. Lauro Enciso por incentivar a pesquisa científica.

Aos meus amigos da Projete Liberdade Capoeira com os quais faço atividade esportiva e cultural, especialmente ao Mestre Gladson, o Contra-Mestre Vinicius, aos Professores Ivan, Gustavo, Stefano e também aos monitores Roseane, Leonardo, Lázaro, Filipe, Mamede, Valquíria, Lucas, Raymy e aos meus amigos de treino: Gabriela, Elaine, Vitoria, Giovana, Natalia, Luis, Karinne, Julia, Andre, Fabiano, Fabiana, Lígia, Daniel, Julio, Pedro, Cristina, Geraldo, Mariela, e principalmente ao CEPEUSP pelo espaço esportivo.

Aos meus amigos de Cusco da faculdade e da escola por estar sempre presentes em todos os momentos, ao meu povo de Ollantaytambo pela inspiração. 
A minha família, principalmente a minha mãe Maria Luisa Bejar Caceres, as minhas irmãs Nery e Jenny, aos meus cunhados Fernando e Cesar, as minhas sobrinhas Vania, Yvaine e ao bebezinho que nascerá, pelo seu apoio, motivação e incentivo.

Finalmente quero agradecer a Deus por ter me dado força e perseverança para continuar neste caminho da ciência da computação. 


\section{Resumo}

\section{CCACYAHUILLCA BEJAR, HANS H. Conexidade Fuzzy Relativa em Grafos Dirigidos e}

sua Aplicação em um Método Híbrido para Segmentação Interativa de Imagens. 2016. 78 p. Dissertação (Mestrado) - Instituto de Matemática e Estatística, Universidade de São Paulo, São Paulo, 2016.

A segmentação de imagens consiste em dividir uma imagem em regiões ou objetos que a compõem, como, por exemplo, para isolar os pixels de um objeto alvo de uma dada aplicação. Em segmentação de imagens médicas, o objeto de interesse comumente apresenta transições em suas bordas predominantemente do tipo claro para escuro ou escuro para claro. Métodos tradicionais por região, como a conexidade fuzzy relativa (RFC - Relative Fuzzy Connectedness), não distinguem bem entre essas bordas similares com orientações opostas. A especificação da polaridade de contorno pode ajudar a amenizar esse problema, o que requer uma formulação matemática em grafos dirigidos. Uma discussão sobre como incorporar essa propriedade no arcabouço do RFC é apresentada neste trabalho. Uma prova teórica da otimalidade do novo algoritmo, chamado conexidade fuzzy relativa com orientação (ORFC - Oriented Relative Fuzzy Connectedness), em termos de uma função de energia em grafos dirigidos sujeita as restrições de sementes é apresentada, bem como a sua aplicação em poderosos métodos híbridos de segmentação. O método híbrido proposto ORFC\&Graph Cut preserva a robustez do ORFC em relação à escolha de sementes, evitando o problema do viés de encolhimento do método de Corte em Grafo (GC - Graph Cut), e mantém o forte controle do GC no delineamento de contornos de bordas irregulares da imagem. Os métodos propostos são avaliados usando imagens médicas de ressonáncia magnética (RM) e tomografia computadorizada (TC) do cérebro humano e de estudos torácicos.

Palavras-chave: Conexidade fuzzy relativa, transformada imagem-floresta, segmentação por corte em grafo, algoritmos de busca em grafo. 


\section{Abstract}

CCACYAHUILlCA BEJAR, HANS H. Relative Fuzzy Connectedness on Directed Graphs and its Appication in a Hybrid Method for Interactive Image Segmentation. 2016. 78 p. Dissertation (Master of Science) - Institute of Mathematic and Statistic, University of São Paulo, São Paulo, 2016.

Image segmentation consists of dividing an image into its composing regions or objects, for example, to isolate the pixels of a target object of a given application. In segmentation of medical images, the object of interest commonly presents transitions at its border predominantly from bright to dark or dark to bright. Traditional region-based methods of image segmentation, such as Relative Fuzzy Connectedness (RFC), do not distinguish well between similar boundaries with opposite orientations. The specification of the boundary polarity can help to alleviate this problem but this requires a mathematical formulation on directed graphs. A discussion on how to incorporate this property in the RFC framework is presented in this work. A theoretical proof of the optimality of the new algorithm, called Oriented Relative Fuzzy Connectedness (ORFC), in terms of an energy function on directed graphs subject to seed constraints is presented, and its application in powerful hybrid segmentation methods. The hybrid method proposed ORFC\&Graph Cut preserves the robustness of ORFC respect to the seed choice, avoiding the shrinking problem of Graph Cut (GC), and keeps the strong control of the GC in the contour delination of irregular image boundaries. The proposed methods are evaluated using magnetic resonance medical imaging (MR) and computed tomography (CT) of the human brain and thoracic studies.

Keywords: Relative fuzzy connectedness, image foresting transform, graph-cut segmentation, graph search algorithms. 


\section{Sumário}

$\begin{array}{ll}\text { Lista de Abreviaturas } & \text { ix }\end{array}$

Lista de Símbolos $\quad$ xi

Lista de Figuras $\quad$ xiii

Lista de Tabelas $\quad$ xvii

1 Introdução $\quad 1$

1.1 Motivação e Objetivos . . . . . . . . . . . . . . . . . . . . . 3

1.2 Organização do trabalho . . . . . . . . . . . . . . . . . . . . 3

2 Conceitos $\quad 5$

2.1 Noções básicas . . . . . . . . . . . . . . . . . . . . . . . . . . 5

2.1 .1 Imagem digital . . . . . . . . . . . . . . . . . . . . . 5

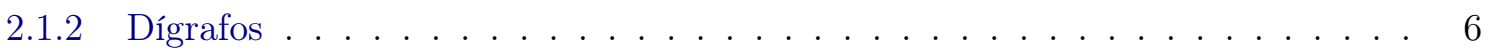

2.2 Grafos a partir de imagens . . . . . . . . . . . . . . . . . . . . 7

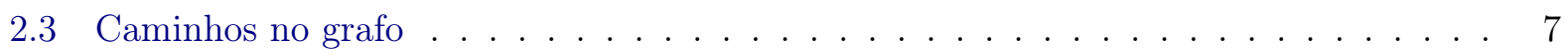

2.4 Segmentação de imagem . . . . . . . . . . . . . . . . . . . . . . 8

2.5 Métricas de avaliação estatística . . . . . . . . . . . . . . . . 8

2.5.1 Matriz de confusão . . . . . . . . . . . . . . . . . . . 8

2.5.2 Coeficiente de similaridade de Dice . . . . . . . . . . . . . . . . . . . 9

3 Métodos relacionados 13

3.1 Transformada imagem-floresta . . . . . . . . . . . . . . . . . . . . . 13

3.1 .1 Função de conexidade . . . . . . . . . . . . . . . . . . . . . . . . 14

3.1 .2 Caminho ótimo . . . . . . . . . . . . . . . . . . . . . . . 14

3.1.3 Floresta de espalhamento como mapa de predecessores . . . . . . . . . . . . . 14

3.1 .4 Algoritmo geral . . . . . . . . . . . . . . . . . . . . . . . 15

3.2 Conexidade fuzzy relativa $\ldots \ldots \ldots \ldots \ldots \ldots \ldots$

3.2.1 A definição original por funções de conexidade . . . . . . . . . . . . . . . 16

3.2.2 Conexidade fuzzy relativa como $\varepsilon_{\infty}$-otimizador $\ldots \ldots \ldots \ldots \ldots$

3.3 Transformada imagem-floresta com orientação . . . . . . . . . . . . . . . . . . . . . 18

3.4 Método de segmentação de corte em grafo . . . . . . . . . . . . . . . . . . . 19

3.4 .1 Problema do fluxo máximo . . . . . . . . . . . . . . . . . . . . 20

3.4 .2 Fluxo no grafo . . . . . . . . . . . . . . . . . . 20 
3.4.3 Corte mínimo em Grafo . . . . . . . . . . . . . . . . . . . . . . . . 21

3.4.4 Arcabouço de segmentação de corte em grafo . . . . . . . . . . . . . . . . . 21

3.4.5 Problema de viés de encolhimento . . . . . . . . . . . . . . . . . 22

4 Conexidade fuzzy relativa com orientação $\quad 23$

4.1 Definição da ORFC por funções de conexidade inversa . . . . . . . . . . . . . . . 23

4.2 Definição ORFC como um corte dirigido no dígrafo . . . . . . . . . . . . . . . . . . . 24

4.3 Algoritmo ORFC baseado em corte no grafo . . . . . . . . . . . . . . . . 25

4.4 Análise dos métodos ORFC e OIFT . . . . . . . . . . . . . . . . . . 30

4.5 Método Híbrido $\mathrm{ORFC}+\mathrm{GC} \ldots \ldots \ldots \ldots \ldots \ldots \ldots$

4.5.1 Características dos métodos ORFC \& GC . . . . . . . . . . . . . . 38

5 Resultados experimentais $\quad 41$

5.1 Análise qualitativa . . . . . . . . . . . . . . . . . . . . . 41

5.2 Análise quantitativa . . . . . . . . . . . . . . . . . 46

6 Conclusões $\quad 51$

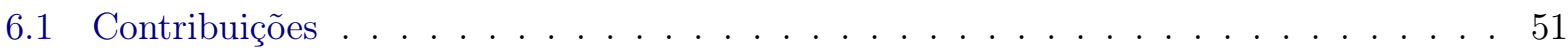

6.2 Dificuldades . . . . . . . . . . . . . . . . . . . . . 52



Referências Bibliográficas $\quad 53$

$\begin{array}{ll}\text { Índice Remissivo } & 58\end{array}$ 


\section{Lista de Abreviaturas}

CAOS Segmentação de Objeto Assistida por Computador (Computer-Aided Object Segmentation)

$\mathrm{CoH} \quad$ Fechamento de buracos (Closing of Holes)

DSC Coeficiente de similaridade de Dice (Dice Similaraty Coefficient)

FC Conexidade Fuzzy (Fuzzy Connectedness)

GC Corte em grafo (Graph Cut)

GGC Corte em Grafo Generalizado (Generalized Graph Cut)

GSC Convexidade Geodésica em Estrela (Geodesic Star Convexity)

IFT Transformada Imagem-Floresta (Image Foresting Transform)

IRFC Conexidade Fuzzy Relativa Iterativa (Iterative Relative Fuzzy Connectedness)

OIFT Transformada Imagem-Floresta Orientada (Oriented Image Foresting Transform)

ORFC Conexidade Fuzzy Relativa Orientada (Oriented Relative Fuzzy Connectedness)

RFC Conexidade Fuzzy Relativa (Relative Fuzzy Connectedness)

RGB Sistema de cores RGB (Red Green Blue)

RM Ressonância Magnética (MR - Magnetic Resonance)

RM-T1 Ressonância Magnética com a sequência ponderada T1 (T1 weighted magnetic resonance image)

TC Tomografía Computadorizada (CT - Computed Tomography) 


\title{
Lista de Símbolos
}

\author{
$\rho \quad$ Constante \\ $\pi \quad$ Caminho \\ $\pi_{a} \quad$ Caminho com destino no pixel $a$ \\ $\pi_{a \rightsquigarrow b} \quad$ Caminho do pixel $a$ ao pixel $b$ \\ $\pi_{\mathcal{S} \rightsquigarrow b} \quad$ Caminho com origem restrita a um conjunto $\mathcal{S}$ \\ $\mathcal{C}_{\text {in }} \quad$ Corte interno \\ $\mathcal{C}_{\text {out }} \quad$ Corte externo \\ $\varepsilon_{\infty} \quad$ Funcional de energia \\ $\varepsilon_{\infty}^{i n} \quad$ Funcional de energia com orientação interna \\ $\varepsilon_{\infty \downarrow}^{i n} \quad$ Mínimo valor do funcional de energia $\varepsilon_{\infty}^{i n}$ \\ $\varepsilon_{\infty}^{\text {out }} \quad$ Funcional de energia com orientação externa \\ $\varepsilon_{\infty \downarrow}^{\text {out }} \quad$ Mínimo valor do funcional de energia $\varepsilon_{\infty}^{\text {out }}$ \\ $\equiv \quad$ Relação de equivalência \\ $f_{\min }^{\nVdash \mathcal{S}} \quad$ Função de conexidade anti-paralela \\ $G \quad$ Grafo dirigido (dígrafo) \\ $G^{T} \quad$ Grafo transposto \\ $\mathcal{V} \quad$ Conjunto de vértices (nós) \\ E Conjunto de arcos ou arestas \\ $w \quad$ Peso de arco \\ $\delta \quad$ Peso de arco não direcionado $(\delta(a, b)=\delta(b, a))$ \\ $E^{T} \quad$ Conjunto de arcos antiparalelos \\ $w^{T} \quad$ Custo de arcos antiparalelos \\ $G_{\leq} \quad$ Grafo residual \\ $\hat{I} \quad$ Imagem digital \\ $\vec{I} \quad$ Mapeamento vetorial da imagem digital \\ I Domínio da imagem \\ $I(a) \quad$ Intensidade do pixel a \\ $K \quad$ Maior diferença de brilho da imagem \\ $V_{b}^{K} \quad$ Mapa de conexidade de fundo com função anti-paralela \\ $V_{o}^{\nVdash} \quad$ Mapa de conexidade de objeto com função anti-paralela \\ $\left[s_{1}\right] \quad$ Núcleo de uma semente $s_{1}$ \\ $\mathcal{S}_{b} \quad$ Conjunto de sementes de fundo da imagem ( $b$ - background) \\ $\mathcal{S}_{o} \quad$ Conjunto de sementes de objeto da imagem (o - object) \\ $G(a) \quad$ Magnitude do gradiente do operador Sobel
}


$\mathcal{X} \quad$ Família de todas as funções $x: V \rightarrow\{0,1\}$

$\chi_{P} \quad$ Função característica do subconjunto $P$

$\hat{x} \quad$ Classificação ideal

$\tilde{\mathcal{X}} \quad$ Família de todas as funções $x: V \rightarrow[0,1]$

$x(a)$ Grau de pertinência do pixel $a$ ao objeto segmentado 


\section{Lista de Figuras}

1.1 Exemplo de segmentação de uma imagem colorida. (a) Imagem de pesos dos arcos do grafo, (b) resultado da IFT apresentando borda irregular e (c) o resultado do $\mathrm{RFC}+\mathrm{GC}$ gerando uma borda mais suave. . . . . . . . . . . . . . . . . . . .

1.2 Exemplo de polaridade de borda em uma imagem médica. (a) Imagem real com bordas ambíguas similares. Não está claro se o objeto de interesse é o endocárdio (no interior) ou o epicárdio (no exterior). (b) Usando um modelo não direcionado é escolhido o endocárdio (no interior), em uma parte da segmentação, e o epicárdio (exterior) na outra parte. (c) No entanto, a informação de polaridade de borda pode ser usada para especificar o alvo como o endocárdio (a borda interna) [Lézoray e Grady

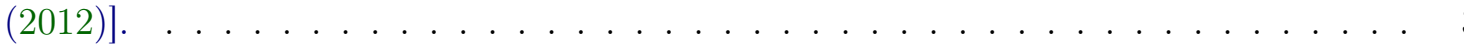

1.3 Exemplo de polaridade de borda em uma imagem sintética onde a denota a semente de objeto e b a semente de fundo. O uso de polaridade é apropriado para especificar o objeto alvo. . . . . . . . . . . . . . . . . . . . . .

2.1 Imagem digital de tamanho $M \times N$ bidimensional $(n=2)$, com três bandas $(m=3)$

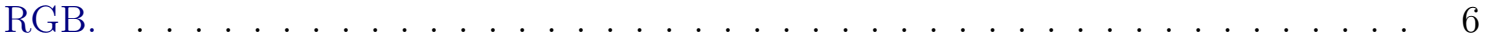

$2.2 G=(\mathcal{V}, E), \operatorname{com} \mathcal{V}=\{a, b, c, d, e, f\}$ e $E=\{\langle a, b\rangle,\langle a, c\rangle,\langle c, a\rangle,\langle b, c\rangle,\langle b, d\rangle,\langle c, d\rangle,\langle d, e\rangle\} . \quad 6$

2.3 Adjacências euclidianas. (a) Imagem 2-D de raio $\rho=1$ (vizinhança-4), (b) raio

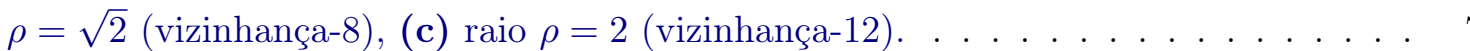

2.4 Exemplo de segmentação de uma imagem em 2 classes. (a) segmentação obtida, (b) segmentação esperada (gabarito) e (c) instâncias segmentadas de forma errada. . . . 9

2.5 O coeficiente de Dice representando a sobreposição espacial e acurácia, onde $D S C=$ $2 \times($ região de interseção $) /($ soma da cardinalidade da região A e da região B). . . . . . 10

2.6 O coeficiente de Dice segundo a matriz de confusão avaliando a classe 1 (objeto). O conjunto A é a segmentação obtida e o conjunto B é a segmentação esperada

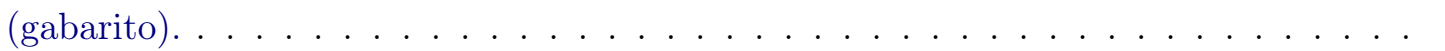

3.1 (a) O mapa de predecessores $P$, armazena os caminhos em ordem inversa. Para um dado nó $b$, seguindo pelo mapa de predecessores chegamos a um nó raiz $R(b)$ tal que $P(R(b))=$ nil. Um caminho $\pi_{b}$ é trivial quando $\pi_{b}=\langle b\rangle$ (isto é, $P(b)=n i l$ ). (b) Duas arvores de espalhamento, uma em vermelho e a outra em azul.

3.2 Exemplo do método IFT. (a) Um grafo com adjacência-4 onde os números indicam pesos dos arcos e os nós $A$ e $B$ são sementes. (b) Uma floresta de caminhos ótimos para $f_{\min }$, quando $H(b)=7$, se $b \in\{A, B\}$ e $H(b)=-\infty$ no caso contrário. . . . . 15 
3.3 Exemplo do RFC seguindo a definição baseada em caminhos. (a) A semente de objeto $\times$, onde $f_{\min }^{\mathcal{S}_{o}}\left(\pi_{\times \rightsquigarrow a}\right)=8, f_{\min }^{\mathcal{S}_{o}}\left(\pi_{\times \rightsquigarrow b}\right)=1, f_{\min }^{\mathcal{S}_{o}}\left(\pi_{\times \rightsquigarrow c}\right)=6$ e $f_{\min }^{\mathcal{S}_{o}}\left(\pi_{\times \rightsquigarrow d}\right)=7$. (b) A semente de fundo $\star$, onde $f_{\text {min }}^{\mathcal{S}_{b}}\left(\pi_{\star \rightsquigarrow d}\right)=8, f_{\min }^{\mathcal{S}_{b}}\left(\pi_{\star \rightsquigarrow b}\right)=1, f_{\min }^{\mathcal{S}_{b}}\left(\pi_{\star \rightsquigarrow c}\right)=6 \mathrm{e}$ $f_{\text {min }}^{\mathcal{S}_{b}}\left(\pi_{\star \rightsquigarrow a}\right)=7$. (c) Resultado de segmentação via $R F C$ mostrada em azul escuro.

3.4 As três soluções acima $(\mathbf{a}, \mathbf{b}, \mathbf{c})$ tem energia ótima $\varepsilon_{\infty}(x)=7$, mas apenas (c) corresponde a uma solução $R F C$ válida de acordo com a Equação 3.6 . . . . . . . . . . . 18

3.5 Exemplo de cálculo do fator de orientação, com valores $w(a, b)$ (Equação 3.8). (a) Grafo não direcionado de uma imagem digital com valores $\delta(a, b)$ indicados na figura. (b) Grafo ponderado dirigido com fator de orientação $\alpha=0.5$. . . . . . . . . . . 19

3.6 Exemplo do resultado de segmentação com o algoritmo OIFT. (a) Algoritmo $A_{O I F T}^{\text {in }}\left(\mathcal{S}_{o}, \mathcal{S}_{b}\right)$

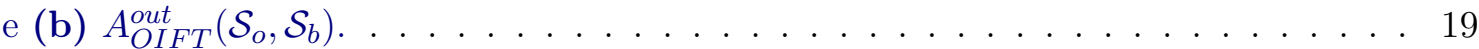

3.7 O fluxo $f$ com valor $|f|=19$. Cada arco $\langle a, b\rangle$ é rotulado por um par de fluxo e capacidade $f(a, b) / c(a, b)$. A notação de barra apenas separa o fluxo e a capacidade; ele não indica divisão.

3.8 Exemplo de viés de encolhimento, onde os pixels em amarelo indicam as sementes internas e as sementes externas são indicadas em roxo. (a-b) Segmentações indesejadas são obtidas quando o número de sementes internas é insuficiente (o objeto delineado colapsa para as sementes internas), (c) resultado da segmentação com um conjunto maior de sementes internas. . . . . . . . . . . . . . . . . . .

4.1 (a) $O R F C$ por funções de conexidade inversa, com a orientação de pixels de escuro para claro $\left(A_{O R F C}^{i n, \rightsquigarrow}\right)$. (b) ORFC como corte dirigido no dígrafo $\left(A_{O R F C}^{i n, \text { se }}\right)$.

4.2 Os cortes externos $\left(\mathcal{C}_{\text {out }}\right)$ e internos $\left(\mathcal{C}_{\text {in }}\right)$ para um objeto candidato mostrando os arcos de saída e de entrada. . . . . . . . . . . . . . . . . . . . . . .

4.3 Exemplo de componente conexo dirigido (DCC). (a) Grafo ponderado dirigido $G=$ $(\mathcal{V}, E, w),(\mathbf{b})$ componente conexo dirigido no grafo $D C C_{G}(x)$.

4.4 Algoritmo $A_{O R F C}^{i n, \diamond<}\left(\mathcal{S}_{o}=\left\{s_{i}\right\}, \mathcal{S}_{b}\right)$. (a) Imagem como dígrafo. (b) Inicialização da IFT com semente de fundo $\mathcal{S}_{b}$ para calcular o valor da conexidade $V_{b}\left(s_{i}\right)$ usando a função de conexidade $f_{\text {min }}^{\mathcal{S}_{b}}$. (c) Resultado da etapa 1: O valor $V_{b}\left(s_{i}\right)=1$ é calculado mediante a IFT. (d) Etapa 2: O grafo $G_{\leq} \cdot(\mathbf{e}, \mathbf{f})$ Etapa 3: a transposta do grafo $G_{\leq}$ e finalmente, os pixels do objeto do DCC.

4.5 Algoritmo $A_{O R F C}^{i n,{ }^{\circ}}\left(\left\{s_{i}\right\}, \mathcal{S}_{b}\right)$. Grafo a partir de uma imagem com semente de objeto $\times$ e semente de fundo $\star$, (a) grafo não dirigido, (b) dígrado com fator de orientação $\alpha=0.5$ (c) resutado do cálculo do mapa de conexidade $V_{b}\left(s_{i}\right)=2$ para uma função de conexidade $f_{\text {min }}^{\mathcal{S}_{b}}$, (d) obtenção do grafo residual $G_{\leq},(\mathbf{e})$ o grafo transposto $G_{\leq}^{T}$, (f) objeto composto pelos pixels que pertencem ao $\operatorname{DCC}\left(s_{i}\right) \ldots \ldots \ldots \ldots$

4.6 Algoritmo $A_{O R F C}^{\text {out,ox }}\left(\left\{s_{i}\right\}, \mathcal{S}_{b}\right)$. Grafo a partir de uma imagem com semente de objeto $\times$ e semente de fundo $\star$, (a) dígrado com fator de orientação $\alpha=0.5$ (b) resutado do cálculo do mapa de conexidade $V_{b}^{\nVdash}\left(s_{i}\right)=2$ para uma função de conexidade $f_{\text {min }}^{\nVdash \mathcal{S}_{b}}$, (c) obtenção do grafo residual $G_{\leq}$, (d) o objeto composto pelos pixels que pertencem

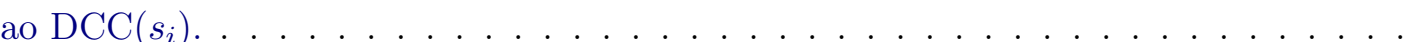


4.7 Exemplo de um grafo direcionado com semente do objeto em diferentes posições, onde $\times$ representa a semente de objeto $\mathrm{e} \star$ a semente de fundo. (a) O resultado da segmentação usando o algoritmo $A_{O R F C}^{\text {out, ’ }}\left(\left\{s_{1}\right\}, \mathcal{S}_{b}\right)$, (b) segmentação usando o algoritmo $A_{O R F C}^{\text {out,se }}\left(\left\{s_{2}\right\}, \mathcal{S}_{b}\right),(\mathbf{c})$ segmentação usando o algoritmo $A_{O R F C}^{\text {out,se }}\left(\left\{s_{1}, s_{2}\right\}, \mathcal{S}_{b}\right)$ com duas sementes no objeto. (d) Resultado de segmentação usando $A_{O I F T}^{\text {out }}\left(\left\{s_{1}\right\}, \mathcal{S}_{b}\right),(\mathbf{e})$ segmentação usando $A_{O I F T}^{\text {out }}\left(\left\{s_{2}\right\}, \mathcal{S}_{b}\right),(\mathbf{f})$ resultado de segmentação usando $A_{O I F T}^{\text {out }}\left(\left\{s_{1}, s_{2}\right\}, \mathcal{S}_{b}\right)$ com duas sementes no objeto. . . . . . . . . . . . . . . . . . . 32

4.8 Exemplo de um grafo direcionado com semente do objeto em diferentes posições, onde $\times$ representa a semente de objeto $\mathrm{e} \star$ a semente de fundo. (a) O resultado da segmentação usando o algoritmo $A_{O R F C}^{\text {out,se }}\left(\left\{s_{1}\right\}, \mathcal{S}_{b}\right)$, (b) segmentação usando o algoritmo $A_{O R F C}^{\text {out,o< }}\left(\left\{s_{2}\right\}, \mathcal{S}_{b}\right),(\mathrm{c})$ segmentação usando o algoritmo $A_{O R F C}^{\text {out,o< }}\left(\left\{s_{1}, s_{2}\right\}, \mathcal{S}_{b}\right)$ com duas sementes no objeto. (d) Resultado de segmentação usando $A_{O I F T}^{\text {out }}\left(\left\{s_{1}\right\}, \mathcal{S}_{b}\right),($ e) segmentação usando $A_{O I F T}^{\text {out }}\left(\left\{s_{2}\right\}, \mathcal{S}_{b}\right)$, (f) resultado de segmentação usando $A_{O I F T}^{\text {out }}\left(\left\{s_{1}, s_{2}\right\}, \mathcal{S}_{b}\right)$ com duas sementes no objeto. . . . . . . . . . . . . . . . . 33

4.9 Exemplo de um grafo direcionado com semente do objeto em diferentes posições, onde $\times$ representa a semente de objeto e $\star$ a semente de fundo. (a) O resultado da segmentação com o algoritmo $A_{O R F C}^{\text {out, sc }}\left(\left\{s_{1}\right\}, \mathcal{S}_{b}\right)$, onde $V_{b}^{K}\left(s_{1}\right)=8$, (b) o algoritmo $A_{O R F C}^{\text {out,se }}\left(\left\{s_{2}\right\}, \mathcal{S}_{b}\right)$ onde $V_{b}^{W}\left(s_{2}\right)=0$ no item (b). (c-d) Os resultados de segmentação com o algoritmo $A_{O I F T}^{\text {out }}\left(\left\{s_{i}\right\}, \mathcal{S}_{b}\right)$. Note que os algoritmos ORFC e OIFT apresentam o mesmo resultado de segmentação. (e) Região de robustez de $s_{1}$ para ORFC, (f)

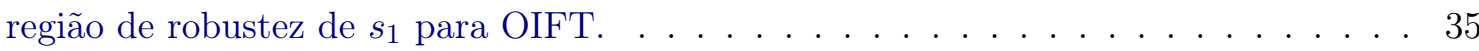

4.10 Exemplo de um grafo direcionado com semente do objeto em diferentes posições, onde $\times$ representa a semente de objeto $\mathrm{e} \star$ a semente de fundo. (a-b) O resultado da segmentação com o algoritmo $A_{O R F C}^{\text {out,se }}\left(\left\{s_{1}\right\}, \mathcal{S}_{b}\right)$, onde $V_{b}^{K}\left(s_{1}\right)=2$ no item (a), e o algoritmo $A_{O R F C}^{\text {out,se }}\left(\left\{s_{2}\right\}, \mathcal{S}_{b}\right)$ onde $V_{b}^{W}\left(s_{2}\right)=2$ no item (b). (c-d) Os resultados de segmentação com o algoritmo $A_{O I F T}^{\text {out }}\left(\left\{s_{i}\right\}, \mathcal{S}_{b}\right) \ldots \ldots \ldots \ldots \ldots$

4.11 Exemplo de um grafo direcionado, onde $\times$ representa a semente de objeto e $\star$ a semente de fundo. (a) O resultado da segmentação usando o algoritmo $A_{O R F C}^{\text {out,ce }}\left(\left\{s_{1}\right\}, \mathcal{S}_{b}\right)$, (b) o núcleo do $\operatorname{ORFC} \mathcal{N}_{O R F C}^{\text {out, se }}\left(\left\{s_{1}\right\}, \mathcal{S}_{b}\right)$, (c) resultado de segmentação usando $A_{O R F C}^{\text {out,oe }}\left(\left\{s_{1}\right\}, \mathcal{S}_{b}\right)$ com fechamento de buraco $(\mathrm{CoH})$, (d) resultado de segmentação usando $A_{O I F T}^{\text {out }}\left(\left\{s_{1}\right\}, \mathcal{S}_{b}\right),\left(\right.$ e) núcleo do OIFT $\mathcal{N}_{O I F T}^{\text {out }}\left(\left\{s_{1}\right\}, \mathcal{S}_{b}\right) \ldots \ldots \ldots$. . . . 37

4.12 Algoritmo $A_{O R F C+G C}^{\text {out,oc }}\left(\mathcal{S}_{o}, \mathcal{S}_{b}\right)$. (a) Imagem de entrada com sementes $\mathcal{S}_{o}$ e $\mathcal{S}_{b}$. (b) $P: \chi_{P}=A_{O R F C}^{\text {out,o< }}\left(\mathcal{S}_{o}, \mathcal{S}_{b}\right)$. (c) $Q: \chi_{Q}=A_{O R F C}^{\text {in, o< }}\left(\mathcal{S}_{b}, \mathcal{S}_{o}\right) .\left(\right.$ d) $A_{G C}^{\text {out }}(P, Q) \ldots \ldots 39$

5.1 Cortes axiais de ressonância magnética do punho. A segmentação de osso rádio por: (a) IRFC [Ciesielski et al. (2007)], (b) OIFT [Miranda e Mansilla (2014)], (c) RFC + GC [Ciesielski et al. (2013)] e (d) ORFC + GC . . . . . . . . . . . . . . . 42

5.2 (a) Um osso tálus em uma fatia de RM de um pé com os marcadores selecionados pelo usuário. (b) A atribuição de peso baseada na imagem conforme proposto em Miranda et al. (2010b). (c-f) Os resultados da segmentação para: (c) IRFC, (d) RFC, (e) OIFT e (f) ORFC. As segmentações aparecem realçadas com sobreposição em amarelo. . . . . . . . . . . . . . . . . . . . . . . . . 
5.3 (a) Uma imagem de TC do fígado com seleção de sementes pelo usuário (b) A atribuição de peso baseada na imagem conforme proposto em Miranda et al. (2010b). (c-d) Resultado de segmentação para: (c) OIFT e (d) ORFC. . . . . . . . . . . . . . 44

5.4 Exemplo de Skull Stripping. (a) O gabarito de segmentação. Os resultados de segmentação para: (b) GC, (c) RFC, (d) ORFC, (e) IRFC, (f) OIFT, (g) RFC+GC e $($ h) $\mathrm{ORFC}+\mathrm{GC} \ldots \ldots \ldots \ldots \ldots \ldots \ldots \ldots$

5.5 Sementes obtidas por erosão de objeto e fundo da segmentação verdadeira (gabarito). 46

5.6 As curvas experimentais para os conjuntos de dados em 2-D. Curva média de acurácia (coeficiente de similaridade de Dice) e as curvas de falsos positivos normalizados, utilizando sementes desigualmente erodidas, para segmentar: (a-b) calcâneo e (c-d)

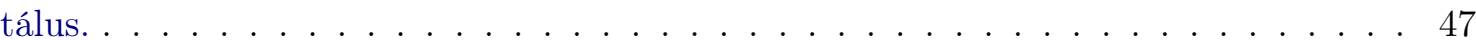

5.7 As curvas experimentais para os conjuntos de dados em 2-D. Curva de tempo de execução, utilizando sementes desigualmente erodidas, para segmentar: (a) calcâneo

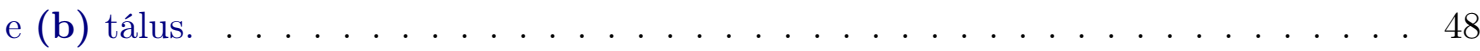

5.8 As curvas experimentais para os conjuntos de dados em 2-D. (a) Curva média de acurácia (coeficiente de similaridade de Dice) e (b) as curvas de falsos positivos normalizados, utilizando sementes desigualmente erodidas, para segmentar o fígado. . 48

5.9 As curvas experimentais para os conjuntos de dados em 2-D. Curva de tempo de execução, utilizando sementes desigualmente erodidas, para segmentar o fígado. . . . 49

5.10 As curvas experimentais para os conjuntos de dados em 3D. Curva média de acurácia (coeficiente de similaridade de Dice) e as curvas de falsos positivos normalizados, utilizando sementes desigualmente erodidas, para segmentar: (a,c,e) o cerebelo e $(\mathrm{b}, \mathrm{d}, \mathbf{f})$ o cérebro completo. . . . . . . . . . . . . . . . . . . 50 


\section{Lista de Tabelas}

2.1 Matriz de confusão gerada apartir de um conjunto de instâncias. . . . . . . . . . . . . 9

2.2 Matriz de confusão normalizada do exemplo da Figura 2.4 . . . . . . . . . . . . . 10 


\section{Capítulo 1}

\section{Introdução}

A segmentação de imagens, como a extração dos pixels de um objeto de interesse presente em uma imagem, é um dos problemas mais desafiadores em processamento de imagens e visão computacional, que é muito útil em várias aplicações, tal como na análise de imagens médicas e biológicas, e na composição de fotos digitais em publicidade [Lézoray e Grady (2012)].

Em algoritmos modernos de processamento de imagens, métodos baseados em grafos têm sido utilizados com sucesso em varias aplicações, servindo como uma ferramenta unificada para representar, processar e analisar imagens [Camilus e Govindan (2012); Lézoray e Grady (2012)]. O uso de grafos é adequado para representar qualquer dado discreto, como imagens digitais, modelando relações de vizinhança. Uma classe importante dos métodos de segmentação de imagem baseados em grafos compreende os métodos interativos baseados em sementes, incluindo diferentes arcabouços, tais como bacia hidrográfica (WS - Watershed) [Cousty et al. (2010); Lotufo et al. (2002)], passeios aleatórios (RW - Random Walks) [Grady (2006)], conexidade fuzzy (FC - Fuzzy Connectedness) [Audigier e Lotufo (2007); Ciesielski et al. (2007); Saha e Udupa (2001)], corte em grafo (GC - Graph Cut) [Boykov e Funka-Lea (2006)], corte de distância (DC - Distance Cut) [Bai e Sapiro (2007)], transformada imagem-floresta (IFT - Image Foresting Transform) [Falcão et al. (2004)] e Corte Crescente (GrowCut) [Vezhnevets e Konouchine (2005)]. O estudo da relação entre diferentes arcabouços, incluindo comparações teóricas e empíricas, tem uma vasta literatura [C.Couprie et al. (2010); Ciesielski et al. (2012a); Miranda e Falcão (2009, 2011); Sinop e L.Grady (2007)], que permitiu que muitos algoritmos (por exemplo, GC, DC, IFT e WS) fossem descritos de uma forma unificada de acordo com um arcabouço comum, que referimos como Corte em Grafo Generalizado (GGC - Generalized Graph Cut) [C.Couprie et al. (2010); Ciesielski et al. (2012b)]. Neste arcabouço, existem duas classes importantes de formulações de energia, os problemas de minimização $\varepsilon_{1}$ e $\varepsilon_{\infty}$ (bem como os algoritmos associados), como discutido em Ciesielski et al. (2012b).

Por outro lado, métodos híbridos para segmentação de imagens são um enfoque recente, os quais combinam duas ou mais técnicas de modo a aproveitar as vantagens individuais delas. Por exemplo, algoritmos baseados puramente na imagem podem ser combinados com abordagens baseadas em modelos de aparência/forma, em uma tentativa de superar algumas deficiências particulares das abordagens, tal como no método híbrido proposto por Udupa et al. (2014). Outro exemplo consiste em explorar as forças complementares de abordagens baseadas em restrições por região (marcadores/sementes) com abordagens de perseguição de borda, como na abordagem interativa do Live Markers [Vallin Spina et al. (2014)], onde segmentos ótimos de borda entre pontos de ancoragem são transformados em marcadores internos e externos para o delineamento por região.

A sinergia entre diferentes abordagens puramente baseadas nas características da imagem também tem sido alvo de estudos. No trabalho de Gulshan et al. (2010), uma segmentação interativa é obtida combinando uma minimização de energia via o algoritmo do Min-Cut/Max-Flow 
[Boykov e Kolmogorov (2004)], com uma restrição de convexidade em estrela geodésica, proveniente da execução do algoritmo de Dijkstra. Em outro artigo [Vicente et al. (2008)], essa mesma técnica de corte em grafo se funde com o algoritmo de Dijkstra, de modo a incorporar restrições de conexidade para superar o problema do "viés de encolhimento" do GC na segmentação de alongamentos finos de objetos, reduzindo consideravelmente a quantidade de interações do usuário necessárias. Já o trabalho de Price et al. (2010) propõe a combinação de uma abordagem de segmentação geodésica com o método de corte em grafo (GC), de modo a aumentar a acurácia. No entanto, os resultados de segmentação por esses métodos apresentam grande sensibilidade em relação a disposição das sementes, dado que as abordagens utilizadas (GC e Dijkstra) são ambas sensíveis às sementes.

Mais recentemente, Ciesielski et al. (2013) combinaram os métodos de conexidade fuzzy relativa (RFC - Relative Fuzzy Connectedness) e o método de corte em grafo (GC - Graph Cut), resultando em um método híbrido que preserva a robustez do RFC em relação à escolha de sementes [Saha e Udupa (2001)] (evitando o problema de encolhimento do GC), e que mantém o forte controle do GC no delineamento de contornos de bordas mal definidas, gerando contornos mais suaves (Figura 1.1).

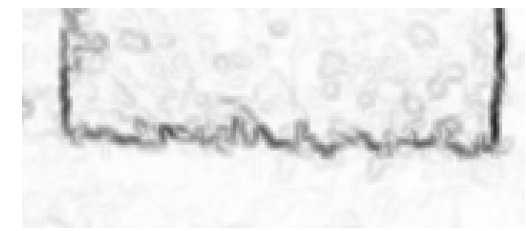

(a)



(b)

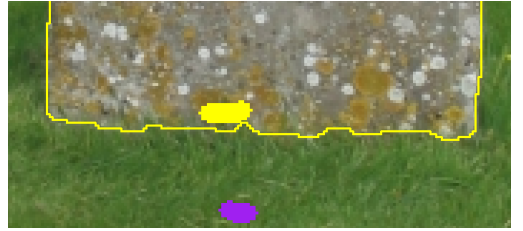

(c)

Figura 1.1: Exemplo de segmentação de uma imagem colorida. (a) Imagem de pesos dos arcos do grafo, (b) resultado da IFT apresentando borda irregular e (c) o resultado do $R F C+G C$ gerando uma borda mais suave.

Nesta pesquisa pretendemos estender esse método híbrido para grafos dirigidos e adicionar a característica de polaridade de borda/orientação [Boykov e Funka-Lea (2006); Miranda e Mansilla (2014); Singaraju et al. (2008)], tal como feito no artigo da transformada imagem-floresta orientada (OIFT - Oriented Image Foresting Transform) [Miranda e Mansilla (2014)].

A polaridade de borda/orientação é uma característica muito importante que explora a informação da borda do objeto (Figura 1.2), em grafos direcionados [Lézoray e Grady (2012)], onde cada aresta não dirigida $\langle a, b\rangle$ é substituída por dois arcos direcionados $\langle a, b\rangle$ e $\langle b, a\rangle$ tal que $w(a, b)$ e $w(b, a)$ podem não ser iguais. A polaridade de borda tem sido tradicionalmente explorada com sucesso por métodos de perseguição de borda, tais como Live Wire [Falcão et al. (1998); Mortensen e Barrett (1998)] e Riverbed [Miranda et al. (2012)]. Mais recentemente, Miranda e Mansilla (2014) consideraram a polaridade de borda na segmentação por região via IFT, onde uma medida regular de dissimilaridade entre pixels vizinhos é multiplicada por um fator de orientação $(1+\alpha$ ou $1-\alpha)$, favorecendo a segmentação de objetos com transições de pixels de claro para escuro como na Figura 1.3b (considerando cortes externos) ou de escuro para claro como na Figura 1.3c (considerando cortes internos) sobre a borda. 


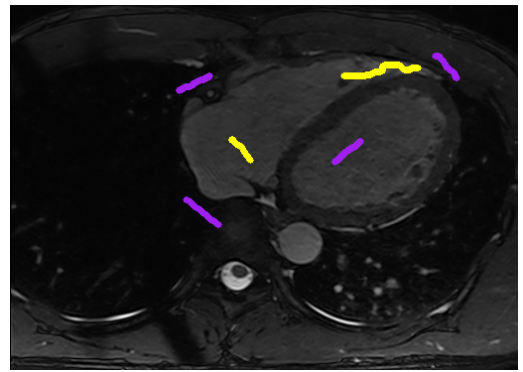

(a) Imagem médica real

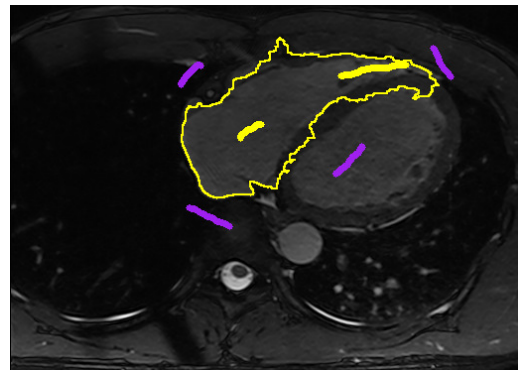

(b) Sem polaridade



(c) Com polaridade

Figura 1.2: Exemplo de polaridade de borda em uma imagem médica. (a) Imagem real com bordas ambíguas similares. Não está claro se o objeto de interesse é o endocárdio (no interior) ou o epicárdio (no exterior). (b) Usando um modelo não direcionado é escolhido o endocárdio (no interior), em uma parte da segmentação, e o epicárdio (exterior) na outra parte. (c) No entanto, a informação de polaridade de borda pode ser usada para especificar o alvo como o endocárdio (a borda interna) [Lézoray e Grady (2012)].

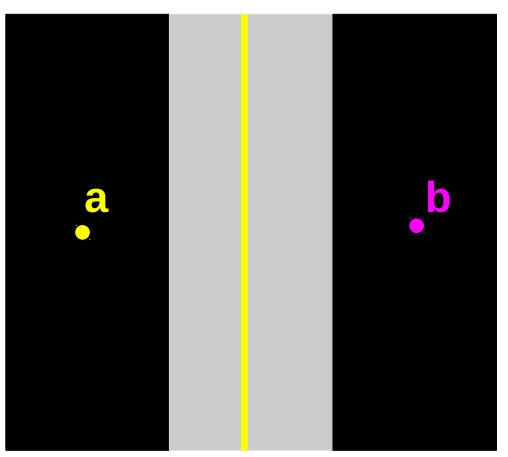

(a) Não dirigido

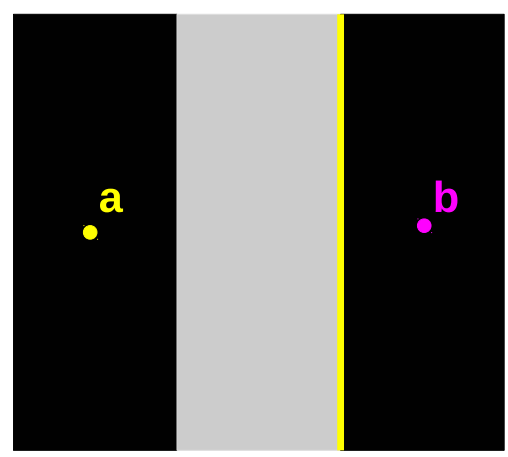

(b) Claro para escuro

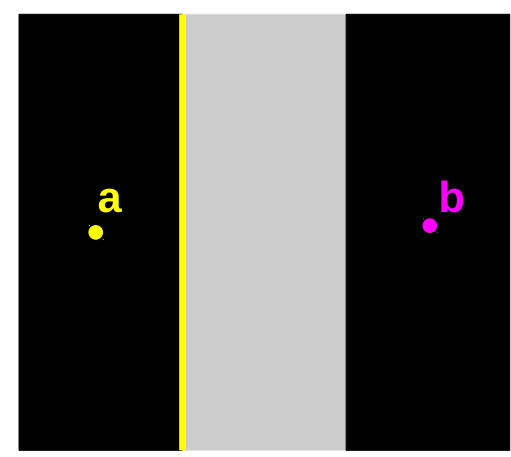

(c) Escuro para claro

Figura 1.3: Exemplo de polaridade de borda em uma imagem sintética onde a denota a semente de objeto e b a semente de fundo. O uso de polaridade é apropriado para especificar o objeto alvo.

\subsection{Motivação e Objetivos}

Os objetivos desse trabalho são:

- Estender o método RFC [Ciesielski e Udupa (2011); Ciesielski et al. (2012a); Saha e Udupa (2001); Udupa et al. (2002)] para grafos direcionados, incorporando a polaridade de borda.

- Utilizando o resultado anterior, estender o método híbrido RFC\&GC [Ciesielski et al. (2013)] para grafos direcionados.

- Validar os métodos realizando comparações com outros métodos da literatura, utilizando bases de dados de imagens médicas 2-D e 3-D.

Os resultados referentes ao primeiro objetivo foram publicados na conferência do SIBGRAPI 2014 [Bejar e Miranda (2014)] e os resultados do método híbrido foram publicados na revista JIVP [Ccacyahuillca Bejar e Miranda (2015)].

\subsection{Organização do trabalho}

No Capítulo 2 fornecemos informações básicas sobre imagem digital, imagens como grafos, segmentação de imagem e a métrica de avaliação. No Capítulo 3 são descritos os métodos relacionados 
usados para desenvolver o projeto, tais como IFT [Falcão et al. (2004)], RFC [Udupa et al. (2002)], GC [Boykov e Funka-Lea (2006)] e OIFT [Miranda e Mansilla (2014)]. Nos demais capítulos são apresentadas as contribuições do presente trabalho, no Capítulo 4 adicionamos a característica de polaridade de borda no método RFC usando grafos dirigidos, incluindo uma prova teórica da otimalidade do novo algoritmo proposto, bem como algumas relações teóricas com outras abordagens. No Capítulo ?? é apresentado o objetivo principal do projeto, onde são fundidos o nosso novo método ORFC [Bejar e Miranda (2014)] e o método GC [Boykov e Funka-Lea (2006)] para grafos dirigidos. No Capítulo 5 é apresentado os resultados experimentais qualitativos e quantitativos, utilizando a técnica do raio de erosão e dilatação. Finalmente, no Capítulo 6 discutimos algumas conclusões obtidas neste trabalho. 


\section{Capítulo 2}

\section{Conceitos}

\subsection{Noções básicas}

\subsubsection{Imagem digital}

Uma imagem digital genérica $\hat{I}$ é um $\operatorname{par}(\mathcal{I}, \vec{I})$ onde $\mathcal{I}$ é um conjunto de pontos do $Z^{n}$ (domínio da imagem) denominados pixels ${ }^{1}$ e $\vec{I}(a)$ é um mapeamento vetorial que associa a cada pixel $a \in \mathcal{I}$ um vetor $\vec{I}(a)=\left\{I_{1}(a), I_{2}(a), \ldots, I_{m}(a)\right\} \in Z^{m}$ de valores escalares, associados a um conjunto de $m$ propriedades físicas. $\mathrm{O}$ valor de $n$ refere-se à dimensão da imagem e o valor de $m$ ao número de bandas. Segundo os parâmetros de uma imagem digital, temos outras definições:

\section{A) Imagem em tons de cinza}

Uma imagem $\hat{I}=(\mathcal{I}, I)$ em tons de cinza (por exemplo, foto preto e branco, imagem de ultrasom, fatia tomográfica) e bidimensional $\left(\mathcal{I} \subset Z^{2}\right)$ possui apenas uma banda $I(m=1)$. A imagem em tons de cinza bidimensional é portanto uma matriz de tamanho $N \times M$ pixels ( $N$ linhas e $M$ colunas). Se $I(a) \in\{0,1\}, \forall a \in \mathcal{I}, \hat{I}$ é dita binária.

\section{B) Imagem multidimensional}

Uma imagem $\hat{I}=(\mathcal{I}, I)$ em tons de cinza e multidimensional define o domínio de amostragem $\mathcal{I} \subset Z^{n}$, para $n>2$. Por exemplo, uma sequência espacial de fatias tomográficas é uma imagem tridimensional $(n=3)$, e uma sequência espacial e temporal de fatias tomográficas é uma imagem tetradimensional $(n=4)$. No primeiro caso, os elementos de $\mathcal{I}$ são chamados de voxels (volume element) e a imagem é chamada de volume.

\section{C) Imagem multibanda}

Uma imagem $\hat{I}=(\mathcal{I}, \vec{I})$ é multibanda quando o mapeamento vetorial $\vec{I}$ associa a cada pixel $a$ obrigatoriamente mais de um valor escalar $\left(\vec{I}(a)=\left\{I_{1}(a), I_{2}(a), \ldots, I_{m}(a)\right\}\right.$, para $\left.m>1\right)$. Imagens de satélite, por exemplo, possuem bandas que correspondem a imagens cinza obtidas nos comprimentos de onda do azul, vermelho, verde, infravermelho, infravermelho próximo, termal, e ultravioleta. O intervalo de amostragem define a resolução espectral. No caso de uma foto colorida temos $m=1,2,3$ correspondendo aos componentes vermelho, verde e azul (Figura 2.1). Observe que o vídeo colorido é uma imagem multidimensional e multibanda.

\footnotetext{
${ }^{1}$ pixel: É o elemento espacial em uma imagem 2-D, para uma imagem 3-D é chamado voxel; de forma geral é conhecido como spel - spatial element; para o melhor entendimento do trabalho as definições estão baseadas em imagens 2-D
} 


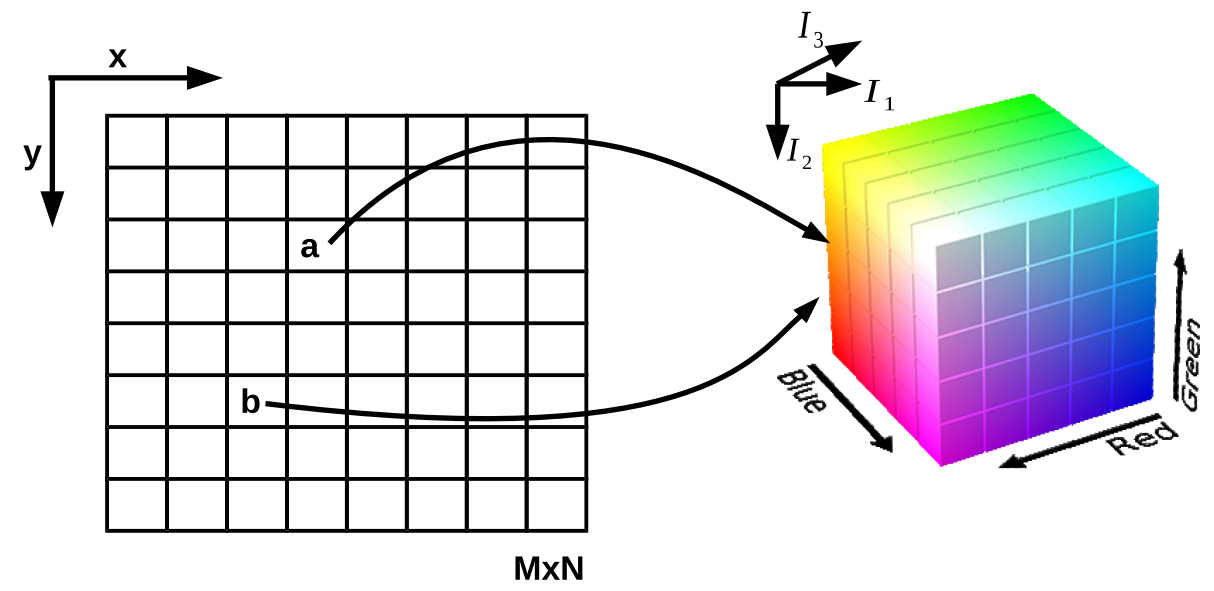

Figura 2.1: Imagem digital de tamanho $M \times N$ bidimensional $(n=2)$, com três bandas $(m=3) R G B$.

\subsubsection{Dígrafos}

Um grafo dirigido ou dígrafo $G=(\mathcal{V}, E)$ é um par ordenado de conjuntos disjuntos, tal que $E \subseteq \mathcal{V} \times \mathcal{V}$ e $\mathcal{V} \neq \varnothing$. O conjunto $\mathcal{V}$ é o conjunto de vértices e $E$ é o conjunto de arcos. Para dois vértices de $G, a$ e $b$, existe no máximo um arco $\langle a, b\rangle$, de $a$ para $b$. Arcos com direções opostas são chamados de arcos anti-paralelos, como por exemplo os $\operatorname{arcos}\langle a, c\rangle$ e $\langle c, a\rangle$ na Figura 2.2.

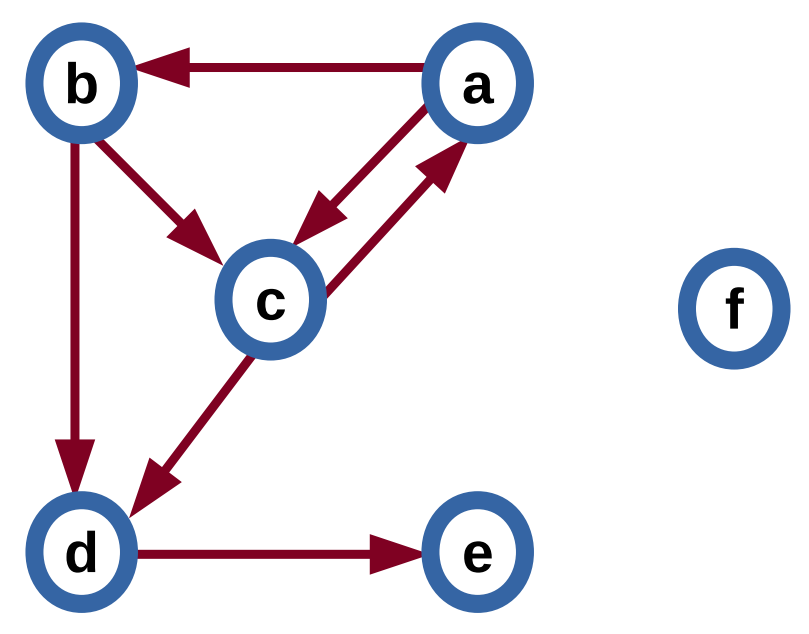

Figura 2.2: $G=(\mathcal{V}, E), \operatorname{com} \mathcal{V}=\{a, b, c, d, e, f\}$ e $E=\{\langle a, b\rangle,\langle a, c\rangle,\langle c, a\rangle,\langle b, c\rangle,\langle b, d\rangle,\langle c, d\rangle,\langle d, e\rangle\}$.

- Um dígrafo $G$ é simétrico se $\forall a, b \in \mathcal{V}, a \neq b,\langle a, b\rangle \in E$ se e somente se $\langle b, a\rangle \in E$.

- Um dígrafo $G$ cujos arcos $\langle a, b\rangle$ possuem valores reais associados (nomeados pesos) dados por uma função $w(a, b)$, é denominado dígrafo ponderado, sendo denotado por uma terna ordenada $G=(\mathcal{V}, E, w)$.

- Um dígrafo simétrico é ponderado com pesos não dirigidos se $w(a, b)=w(b, a)$ para todo $\langle a, b\rangle \in E$, caso contrário, temos um dígrafo ponderado de modo dirigido.

- A transposta $G^{T}=\left(\mathcal{V}, E^{T}, w^{T}\right)$ de um dígrafo ponderado $G=(\mathcal{V}, E, w)$ é o único dígrafo ponderado sobre o mesmo conjunto de vértices $\mathcal{V}$ com todos os arcos invertidos em comparação com a orientação dos arcos correspondentes em $G$ (isto é, para qualquer dos seus $\operatorname{arcos}\langle a, b\rangle \in$ $E^{T}$, o par $\langle b, a\rangle$ é um arco de $G$, e $\left.w^{T}(a, b)=w(b, a)\right)$. 
- Um dígrafo ponderado $G$ é simétrico ponderado de modo não dirigido se $G$ é igual à sua transposta $G^{T}$.

\subsection{Grafos a partir de imagens}

Uma imagem pode ser interpretada como um dígrafo ponderado $G=(\mathcal{V}, E, w)$ cujos nós $\mathcal{V}$ são os pixels da imagem no seu domínio $\mathcal{I} \subset Z^{n}$, e cujos arcos são os pares ordenados de pixels $\langle a, b\rangle \in E$. Por exemplo, pode-se tomar $E$ consistindo de todos os pares de pixels $\langle a, b\rangle$ no produto Cartesiano $\mathcal{I} \times \mathcal{I}$ tal que $d(a, b) \leq \rho$ e $a \neq b$, onde $d(a, b)$ denota a distância Euclidiana e $\rho$ é uma constante especificada (por exemplo: vizinhança-4, quando $\rho=1$, e vizinhança-8 quando $\rho=\sqrt{2}$, no caso de imagens 2-D). Essa forma de definir os arcos é conhecida como relação de adjacência circular (Figura 2.3).

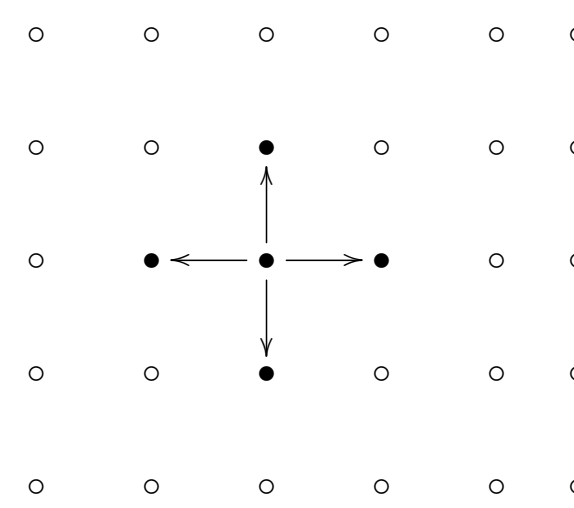

(a)

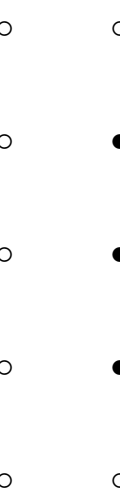

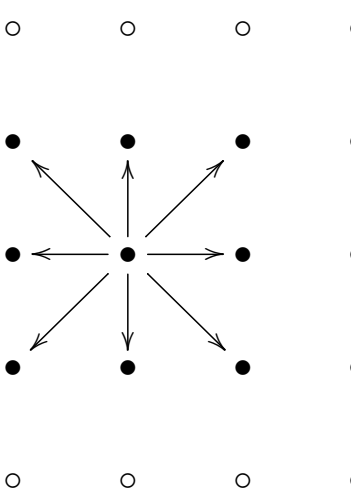

(b)

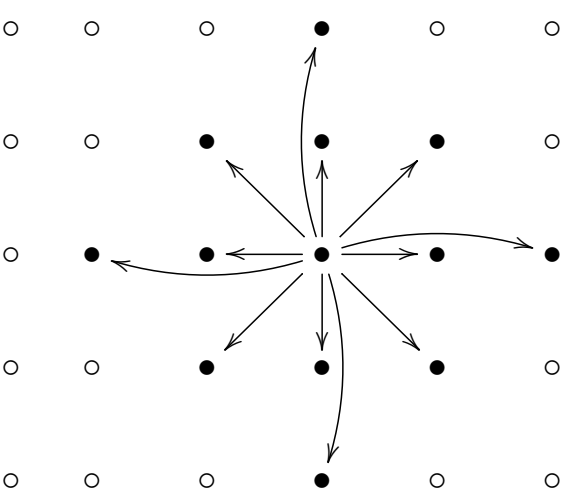

(c)

Figura 2.3: Adjacências euclidianas. (a) Imagem 2-D de raio $\rho=1$ (vizinhança-4), (b) raio $\rho=\sqrt{2}$ (vizinhança-8), (c) raio $\rho=2$ (vizinhança-12).

Outros tipos de relação de adjacência são:

- Retangular: $\langle a, b\rangle \in E$ se $\left|x_{b}-x_{a}\right| \leq \frac{h}{2}$ e $\left|y_{b}-y_{a}\right| \leq \frac{j}{2}$, onde $h$ e $j$ são os comprimentos dos lados do retângulo com centro em $\left(x_{a}, y_{a}\right)$.

- Baseado em conjunto: $\langle a, b\rangle \in E$ se $b-a \in\{(-1,-1),(1,-1)\}$.

- Baseada em propriedades locais: $\langle a, b\rangle \in E$ se $d(a, b) \leq \rho_{i}$ e $d(\vec{I}(a), \vec{I}(b)) \leq \rho_{j}$, onde $d()$ é o operador de distância Euclideana e $\rho_{i}$ e $\rho_{j}$ são os raios nos espaços da imagem e de atributos (paramétrico), respectivamente.

Cada arco $\langle a, b\rangle \in E$ tem um peso fixo $w(a, b) \geq 0$, entre pixels vizinhos, que idealmente deve ser projetado para ter valores baixos nas transições da borda do objeto de interesse (por exemplo, $w(a, b)=K-|I(a)-I(b)|$, onde $K$ é a maior diferença de brilho para uma imagem com uma única banda, com valores dados por $I(a))$.

\subsection{Caminhos no grafo}

Para um determinado grafo da imagem $G=(\mathcal{V}, E, w)$, utilizamos o conceito de caminho no grafo representado por $\pi$, além disso existem outras formas especificas de representar um caminho, como por exemplo: 
A) $\pi_{a}$ : Um caminho $\pi_{a}=\left\langle t_{1}, t_{2}, \ldots, t_{n}=a\right\rangle$ é uma sequência de pixels adjacentes (isto é, $\left\langle t_{i}, t_{i+1}\right\rangle \in$ $E, i=1, \ldots, n-1)$ com um pixel de destino $a$. Um caminho é trivial quando $\pi_{a}=\langle a\rangle$. Um caminho $\pi_{b}=\pi_{a} \cdot\langle a, b\rangle$ indica a extensão de um caminho $\pi_{a}$ por um arco $\langle a, b\rangle$.

B) $\pi_{a \rightsquigarrow b}$ : Quando queremos indicar explicitamente a origem de um caminho, a notação $\pi_{a \rightsquigarrow b}=$ $\left\langle t_{1}=a, t_{2}, \ldots, t_{n}=b\right\rangle$ pode também ser utilizada, onde $a$ representa a origem e $b$ o nó de destino. De modo mais geral, pode-se usar $\pi_{\mathcal{S} \leadsto b}=\left\langle t_{1}, t_{2}, \ldots, t_{n}=b\right\rangle$ para indicar um caminho com origem restrita a um conjunto $\mathcal{S}$ (isto é, $t_{1} \in \mathcal{S}$ ).

Um dígrafo é dito ser fortemente conexo se há um caminho de cada vértice para todos os outros vértices.

\subsection{Segmentação de imagem}

A segmentação de imagem pode ser vista como um problema de classificação dos pixels em diferentes classes. Neste trabalho, vamos considerar a segmentação em duas classes, objeto e fundo. A segmentação pode ser rígida ou fuzzy.

Seja o dígrafo ponderado $G=(\mathcal{V}, E, w)$, onde $\mathcal{V}$ é o conjunto finito de vértices. Considere o espaço $\tilde{\mathcal{X}}$ de todas as funções $x: \mathcal{V} \rightarrow[0,1]$, denominado subconjuntos fuzzy de $\mathcal{V}$, com o valor $x(a)$ indicando um grau de pertinência com o qual a pertence ao subconjunto (objeto segmentado).

Seja $\mathcal{X}$ a família de todas as funções $x \in \tilde{\mathcal{X}}$ com apenas os valores permitidos de 0 e 1 (isto é, $x: \mathcal{V} \rightarrow\{0,1\}$ ), referida como a família de todos subconjuntos rígidos (hard) de $\mathcal{V}$. Cada $x \in \mathcal{X}$ é identificado com o subconjunto $P=\{a \in \mathcal{V}: x(a)=1\}$ de $\mathcal{V}$. Note que, em tal caso, $x$ é a função característica $\chi_{P}$ de $P \subseteq \mathcal{V}$. Por exemplo, considere o conjunto finito $\mathcal{V}=\left\{t_{1}, t_{2}, t_{3}, t_{4}, t_{5}\right\}$ e o subconjunto $P=\left\{t_{2}, t_{3}, t_{5}\right\}$, então, temos a seguinte função característica $\chi_{P}$

$$
x\left(t_{1}\right)=0, x\left(t_{2}\right)=1, x\left(t_{3}\right)=1, x\left(t_{4}\right)=0, x\left(t_{5}\right)=1
$$

Geralmente restringimos a coleção $\mathcal{X}$ de todos os objetos permitidos indicando dois conjuntos disjuntos, referidos como sementes: $\mathcal{S}_{o} \subset \mathcal{V}$ indicando o objeto e $\mathcal{S}_{b} \subset \mathcal{V}$ indicando o fundo (com $\left.\mathcal{S}_{o} \cap \mathcal{S}_{b}=\emptyset\right)$. Isto limita a coleção de saídas admissíveis do algoritmo para a família $\mathcal{X}\left(\mathcal{S}_{o}, \mathcal{S}_{b}\right)$ de todo $x \in \mathcal{X}$ com $x(a)=1$ para todo $a \in \mathcal{S}_{o}$, e $x(b)=0$ para todo $b \in \mathcal{S}_{b}$. Repare que $\mathcal{X}\left(\mathcal{S}_{o}, \mathcal{S}_{b}\right)=\left\{\chi_{P}: \mathcal{S}_{o} \subseteq P \subseteq \mathcal{V} \backslash \mathcal{S}_{b}\right\}$.

\subsection{Métricas de avaliação estatística}

\subsubsection{Matriz de confusão}

Considera-se um conjunto de amostras, onde uma instância $b$ (elemento que não se sabe a qual classe pertence e deseja-se classificá-lo) pode assumir valores no conjunto $\{0,1\}$, negativo e positivo respectivamente. No contexto de segmentação, temos $b \in \mathcal{I}$ e queremos descobrir a sua classe $x(b)$. Considere $\hat{x}(b): \mathcal{I} \rightarrow\{0,1\}$ como sendo a classificação ideal de uma segmentação perfeita (gabarito).

Se a instância $b$ é positiva $(\hat{x}(b)=1)$ e é classificada como positiva $(x(b)=1)$, conta-se como verdadeira positiva; se é classificada como negativa $(x(b)=0)$, conta-se como falsa negativa. Se a instância é negativa $(\hat{x}(b)=0)$ e é classificada como tal, conta-se como verdadeira negativa; se é classificada como positiva $(x(b)=1)$, conta-se como falsa positiva. Portanto, dado um método de segmentação (classificador) e um conjunto de instâncias pode-se construir uma matriz de confusão de 2 por 2, no caso de 2 classes. Esta matriz serve como base para muitas métricas de avaliação da qualidade da classificação. A Tabela 2.1 mostra a matriz de confusão [Comas e Meschino (2014)]. 


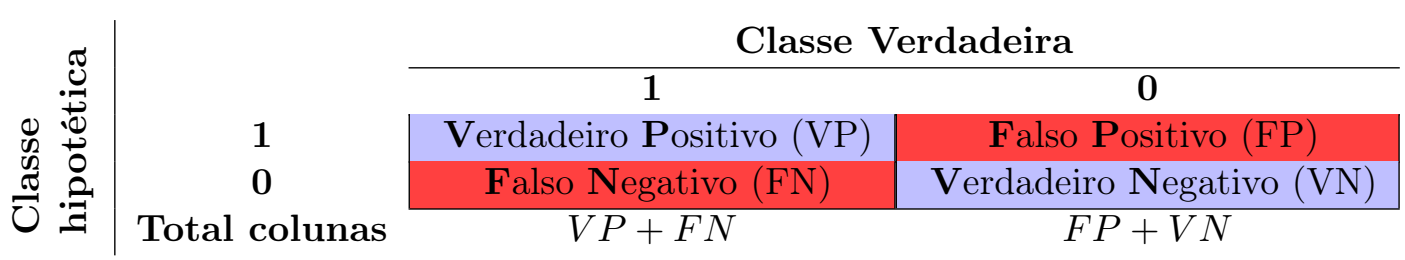

Tabela 2.1: Matriz de confusão gerada apartir de um conjunto de instâncias.

O conjunto de Equações 2.1 demonstra algumas métricas que podem ser calculadas a partir da matriz de confusão, tais como a taxa de acerto (recall ou $\boldsymbol{t p}$ rate), a taxa de falso alarme - ( $\boldsymbol{f} \boldsymbol{p}$ rate), a precisão (precision) e a acurácia (accuracy).

$$
\begin{array}{rlrl}
\text { tprate } & =\frac{V P}{V P+F N} & \text { fprate } & =\frac{F P}{F P+V N} \\
\text { precision } & =\frac{V P}{V P+F P} & \text { recall }=\text { tprate } & =\frac{V P}{V P+F N} \\
\text { accuracy } & =\frac{V P+V N}{V P+F N+F P+V N} &
\end{array}
$$

Na Figura 2.4 um exemplo de uma imagem de $8 \times 8$ pixels é exibida, sendo a imagem segmentada em duas classes (Classe $\mathbf{0}$ em cor cinza e Classe $\mathbf{1}$ em cor amarelo). O número de elementos de cada classe são: Na segmentação obtida $\left|A_{0}\right|=38$ e $\left|A_{1}\right|=42$; e no gabarito $\left|B_{0}\right|=30$ e $\left|B_{1}\right|=50$.

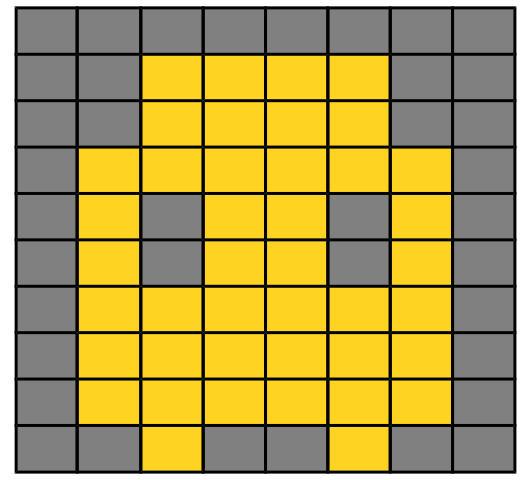

(a)

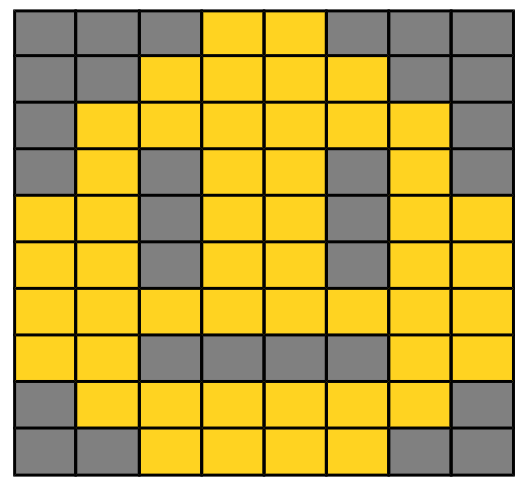

(b)

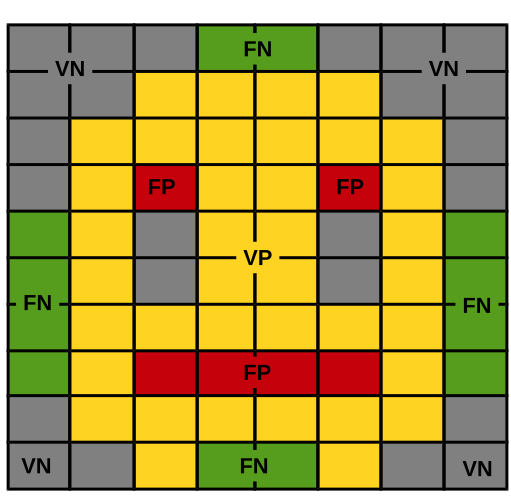

(c)

Figura 2.4: Exemplo de segmentação de uma imagem em 2 classes. (a) segmentação obtida, (b) segmentação esperada (gabarito) e (c) instâncias segmentadas de forma errada.

A matriz de confusão correspondente ao exemplo da Figura 2.4 é apresentada na Tabela 2.2:

\subsubsection{Coeficiente de similaridade de Dice}

O coeficiente de similaridade Dice (DSC) [Comas e Meschino (2014); Dice (1945); Sorensen (1948)] é usado como uma métrica de avaliação estatística da acurácia de desempenho das segmentações de imagens (dados binários), baseado na comparação entre o número de acertos e o número total de elementos dos objetos envolvidos. Pode ser facilmente convertido para coeficiente de dissimilaridade: Se a similaridade for denominada $s$, a medida de dissimilaridade será o seu complementar $(1-s)$. O $D S C$ é a principal métrica de validação para o índice de sobreposição espacial entre 2 imagens binárias [Kalpathy-Cramer e Müller (2011); Meyer (2002)]. O seu valor 


\begin{tabular}{|c|c|c|}
\hline & $\begin{array}{c}\text {...foram classificados } \\
\text { como } 1\end{array}$ & $\begin{array}{c}\text {...foram classificados } \\
\text { como } 0\end{array}$ \\
\hline $\begin{array}{l}\text { Pixels que } \\
\text { pondendo à } \\
1 \ldots\end{array}$ & $\frac{36}{50}=0.72$ & $\frac{14}{50}=0.28$ \\
\hline $\begin{array}{l}\text { Pixels que } \\
\text { pondendo à corres- } \\
0 \ldots\end{array}$ & $\frac{6}{30}=0.2$ & $\frac{24}{30}=0.8$ \\
\hline
\end{tabular}

Tabela 2.2: Matriz de confusão normalizada do exemplo da Figura 2.4.

varia entre 0 (sem sobreposição) e 1 (sobreposição completa), sendo calculado a partir da seguinte fórmula: $D S C=2|A \cap B| /(|A|+|B|)$, onde $A$ representa a saída de uma segmentação particular $(A=\{a \in \mathcal{I}: x(a)=1\}), B$ é o gabarito $(B=\{b \in \mathcal{I}: \hat{x}(b)=1\})$ e " $\cap$ " é a interseção, apresentada na Figura 2.5:

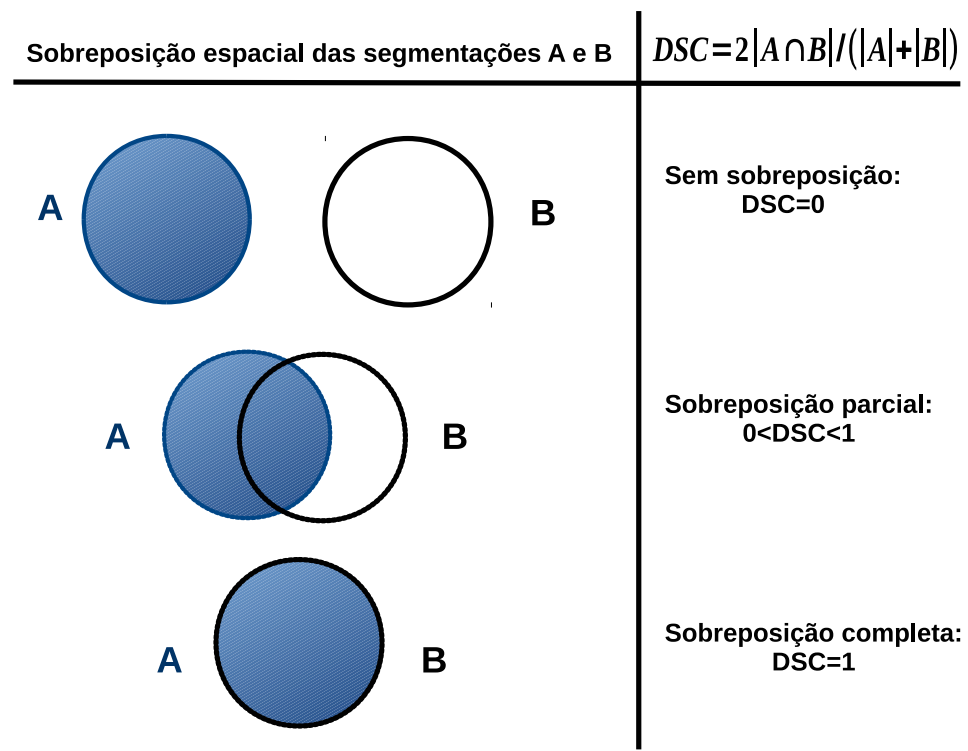

Figura 2.5: $O$ coeficiente de Dice representando a sobreposição espacial e acurácia, onde DSC $=2 \times($ região de interseção)/(soma da cardinalidade da região $A$ e da região B).

Segundo a Figura 2.6 o coeficiente de Dice pode ser também calculado com a equação:

$$
D S C=\frac{2 \times V P}{F P+F N+2 \times V P}
$$

Para o exemplo da Figura 2.4 o cálculo do coeficiente de Dice na classe 1 (pixels amarelos objeto) é:

$$
D S C_{1}=\frac{2 \times 38}{44+50}=0.80
$$




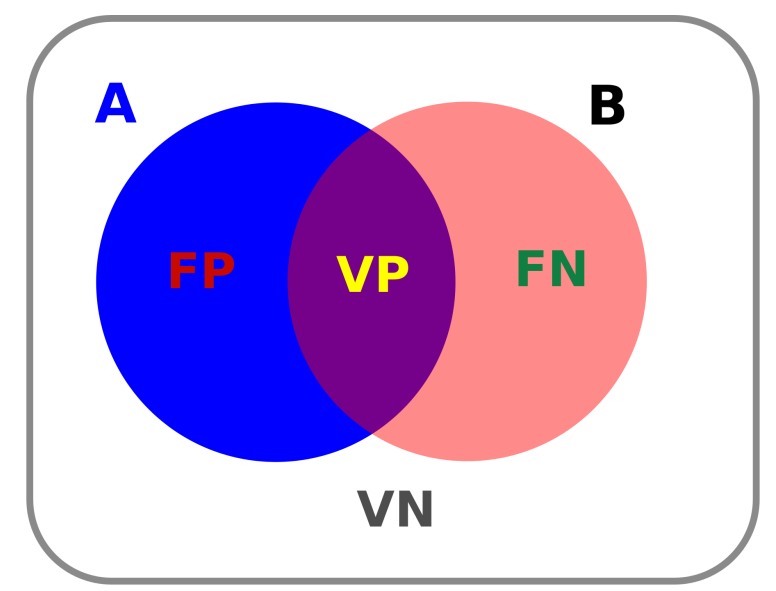

Figura 2.6: $O$ coeficiente de Dice segundo a matriz de confusão avaliando a classe 1 (objeto). O conjunto $\mathbf{A}$ é a segmentação obtida e o conjunto $\mathbf{B}$ é a segmentação esperada (gabarito). 


\section{Capítulo 3}

\section{Métodos relacionados}

Alguns métodos de segmentação de imagem baseados em grafos que estão diretamente relacionados com nosso trabalho serão descritos a seguir.

\subsection{Transformada imagem-floresta}

A transformada imagem-floresta (IFT - Image Foresting Transform) é uma extensão do algoritmo de Dijkstra em grafos que trata o problema da floresta de caminhos ótimos para funções de conexidade mais genéricas, desenvolvida por Falcão et al. (2004).

A IFT trata a imagem como um grafo onde todos os pixels são nós e as arestas são definidas por uma relacão de adjacência entre os pixels vizinhos. Usa-se geralmente a vizinhança-4 (ou vizinhança de quarteirão - city-block) onde cada pixel é considerado adjacente aos pixels pertencentes ao disco euclidiano de raio unitário centrado nele, ou a vizinhança-8 (ou vizinhança de xadrez - chessboard) onde o pixel/nó compartilha arestas com os pixels/nós situados numa distância inferior ou igual a $\sqrt{2}$ (vide Figura 2.3).

A IFT calcula uma partição ótima dos pixels/nós em árvores de custo mínimo. Portanto, cada $\mathrm{pixel} /$ nó recebe um rótulo específico da árvore à qual ele pertence, possui um predecessor na árvore e tem um custo de caminho associado.

A IFT possui as seguintes motivações:

- Unificação: Vários operadores de imagem são derivados de um algoritmo geral. Isto favorece implementações baseadas em hardware [Cappabianco et al. (2007)], facilitando a compreensão das relações entre alguns operadores de imagem, e favorecendo possíveis extensões.

- Eficiência: A maioria dos operadores de imagem podem ser implementados em tempo linear e otimizações adicionais são possíveis com cálculo diferencial [Falcão e Bergo (2004)] e paralelo [Bergo e Falcão (2007)].

- Simplicidade: Os operadores de imagem são reduzidos a escolha de poucos parâmetros no algoritmo da IFT e um processamento local dos atributos da floresta obtida.

Aplicações que podem ser tratadas via IFT:

- Transformadas de distância e operadores relacionados: Transformada da distância euclidiana, esqueleto multiescala, dimensões fractais, filtragem de forma, detecção de saliências de forma, descritores de forma, caminhos geodésicos, [Falcão et al. (2004)].

- Filtragem e segmentação de imagens: Reconstrução morfológica [Falcão et al. (2001)] e segmentação de imagem baseada na transformada de Watershed, Live Wire [Falcão et al. 
(1998)], Riverbed [Miranda et al. (2012)], Growcut por autômato celular [Miranda e Falcão (2011); Vezhnevets e Konouchine (2005)], e métodos de conexidade fuzzy [Ciesielski et al. (2012a)].

- Reconhecimento de padrões: Clustering de dados [Rocha et al. (2009)], e classificação de padrões supervisionada [J.P. Papa e Miranda (2007)].

Neste trabalho, a IFT será apresentada de modo mais específico para o problema da segmentação de imagens.

\subsubsection{Função de conexidade}

Uma função de conexidade $f(\pi)$ associa um valor escalar a qualquer caminho $\pi$ no grafo $G=(\mathcal{V}=\mathcal{I}, E, w)$, com base em propriedades da imagem ao longo deste caminho. Em segmentação, por exemplo, propriedades locais da imagem (vetor de atributos) e globais do objeto desejado (modelo de textura, cor e forma) podem ser exploradas no cálculo de $f\left(\pi_{a \rightsquigarrow b}\right)$ para indicar a força de conexidade entre seus nós terminais $a$ e $b$ através do caminho $\pi_{a \rightsquigarrow b}$. As funções de conexidade são normalmente especificadas por uma regra de inicialização e uma regra de extensão de caminho. Por exemplo considere a função $f_{\text {min }}$ :

$$
\begin{aligned}
f_{\min }(\langle b\rangle) & =H(b) \\
f_{\min }\left(\pi_{a} \cdot\langle a, b\rangle\right) & =\min \left\{f_{\min }\left(\pi_{a}\right), w(a, b)\right\}
\end{aligned}
$$

onde $H(b)$ é um valor inicial para caminhos triviais.

\subsubsection{Caminho ótimo}

Um caminho $\pi_{b}$ é ótimo se $f\left(\pi_{b}\right) \geq f\left(\tau_{b}\right)$ para qualquer outro caminho $\tau_{b}$, independentemente de sua raiz. Para cada nó $b \in \mathcal{I}$, temos um valor único $V(b)$ que armazena o valor de um caminho ótimo com término em $b$ :

$$
V(b)=\max _{\forall \pi_{b} e m}\left\{f\left(\pi_{b}\right)\right\}
$$

\subsubsection{Floresta de espalhamento como mapa de predecessores}

Um mapa de predecessores é um função $P$ que atribui para cada nó $b$ em $\mathcal{I}$ algum outro nó adjacente em $\mathcal{I}$, ou uma marca distintiva nil $\notin \mathcal{I}$, caso em que $b$ é dito ser uma raiz do mapa. Uma floresta de espalhamento é um mapa de predecessores que não contém ciclos, isto é, um mapa que leva cada pixel para nil em um número finito de iterações. Para qualquer pixel $b \in \mathcal{I}$, uma floresta de espalhamento $P$ define um caminho $\pi_{b}^{P}$ recursivamente como $\langle b\rangle$ se $P(b)=n i l$, e $\pi_{a}^{P} \cdot\langle a, b\rangle$ se $P(b)=a \neq n i l$ (Figura 3.1). 


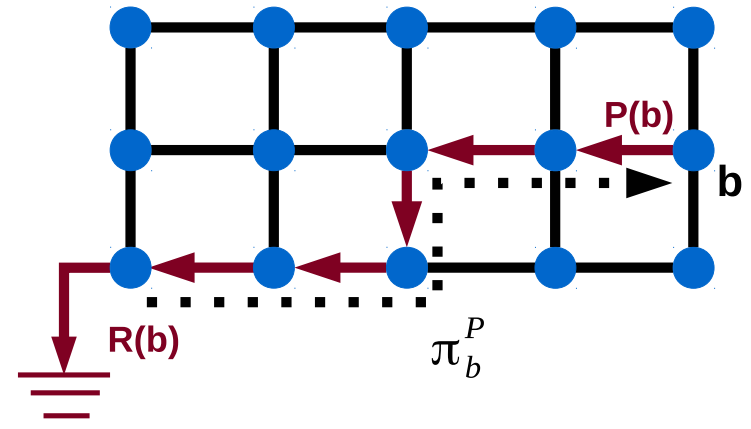

(a)

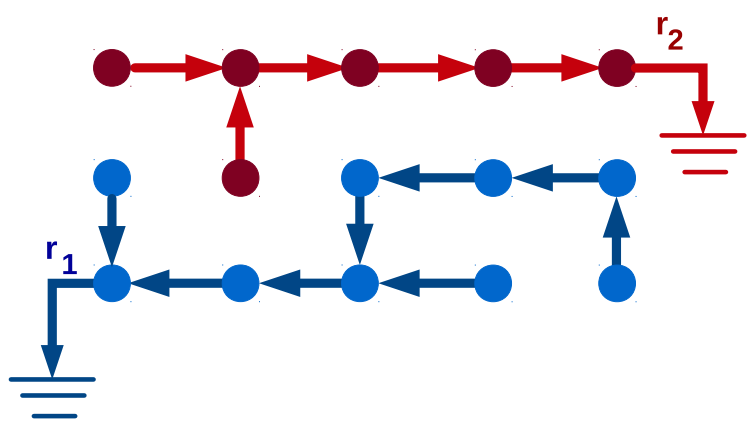

(b)

Figura 3.1: (a) O mapa de predecessores $P$, armazena os caminhos em ordem inversa. Para um dado nó $b$, seguindo pelo mapa de predecessores chegamos a um nó raiz $R(b)$ tal que $P(R(b))=$ nil. Um caminho $\pi_{b}$ é trivial quando $\pi_{b}=\langle b\rangle$ (isto é, $P(b)=$ nil). (b) Duas arvores de espalhamento, uma em vermelho e a outra em azul.

Na IFT, as raízes são os máximos locais da função $V$ de conexidade ótima. Estas raízes competem entre elas e cada raiz conquista os nós mais fortemente conexos a ela do que a qualquer outra, particionando a imagem em uma floresta onde cada raiz forma uma árvore de caminhos ótimos (Figura 3.2).

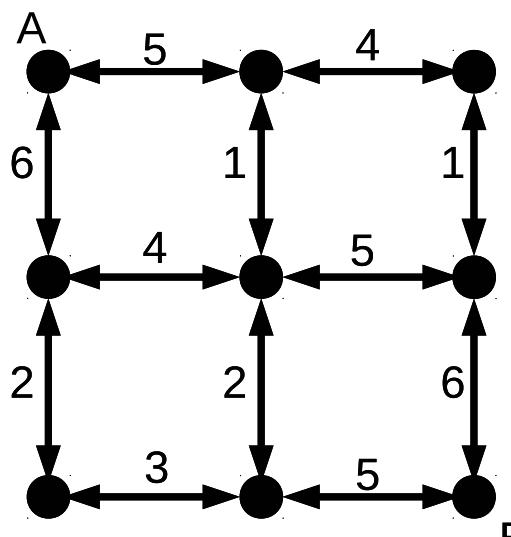

(a)

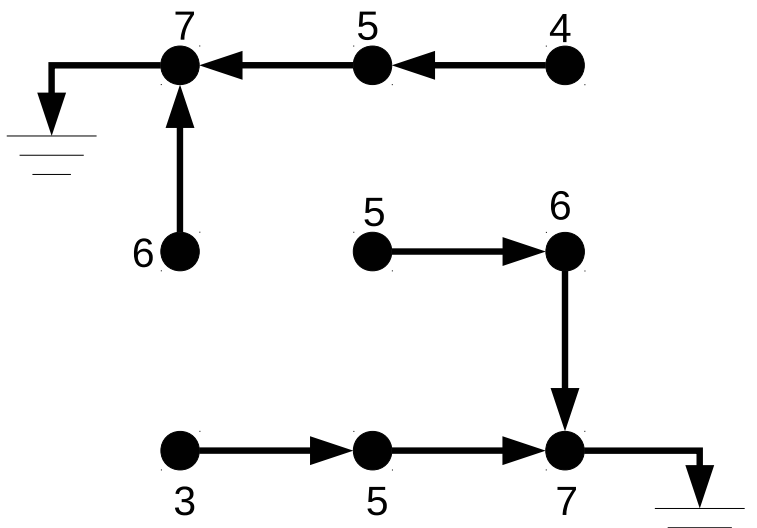

(b)

Figura 3.2: Exemplo do método IFT. (a) Um grafo com adjacência-4 onde os números indicam pesos dos arcos e os nós $A$ e $B$ são sementes. (b) Uma floresta de caminhos ótimos para $f_{\text {min }}$, quando $H(b)=7$, se $b \in\{A, B\}$ e $H(b)=-\infty$ no caso contrário.

\subsubsection{Algoritmo geral}

A IFT essencialmente generaliza o algoritmo de Dijkstra para funções de conexidade, onde para qualquer nó $b \in \mathcal{I}$, existe um caminho ótimo $\pi_{b}$ que é o trivial, ou tem a forma $\pi_{a} \cdot\langle a, b\rangle$ onde:

- (Condição 1) $f\left(\pi_{a}\right) \geq f\left(\pi_{b}\right)$.

- (Condição 2) $\pi_{a}$ é ótimo.

- (Condição 3) para qualquer caminho ótimo $\tau_{a}, f\left(\tau_{a} \cdot\langle a, b\rangle\right)=f\left(\pi_{b}\right)$.

Estas condições são aplicadas apenas a caminhos ótimos. 


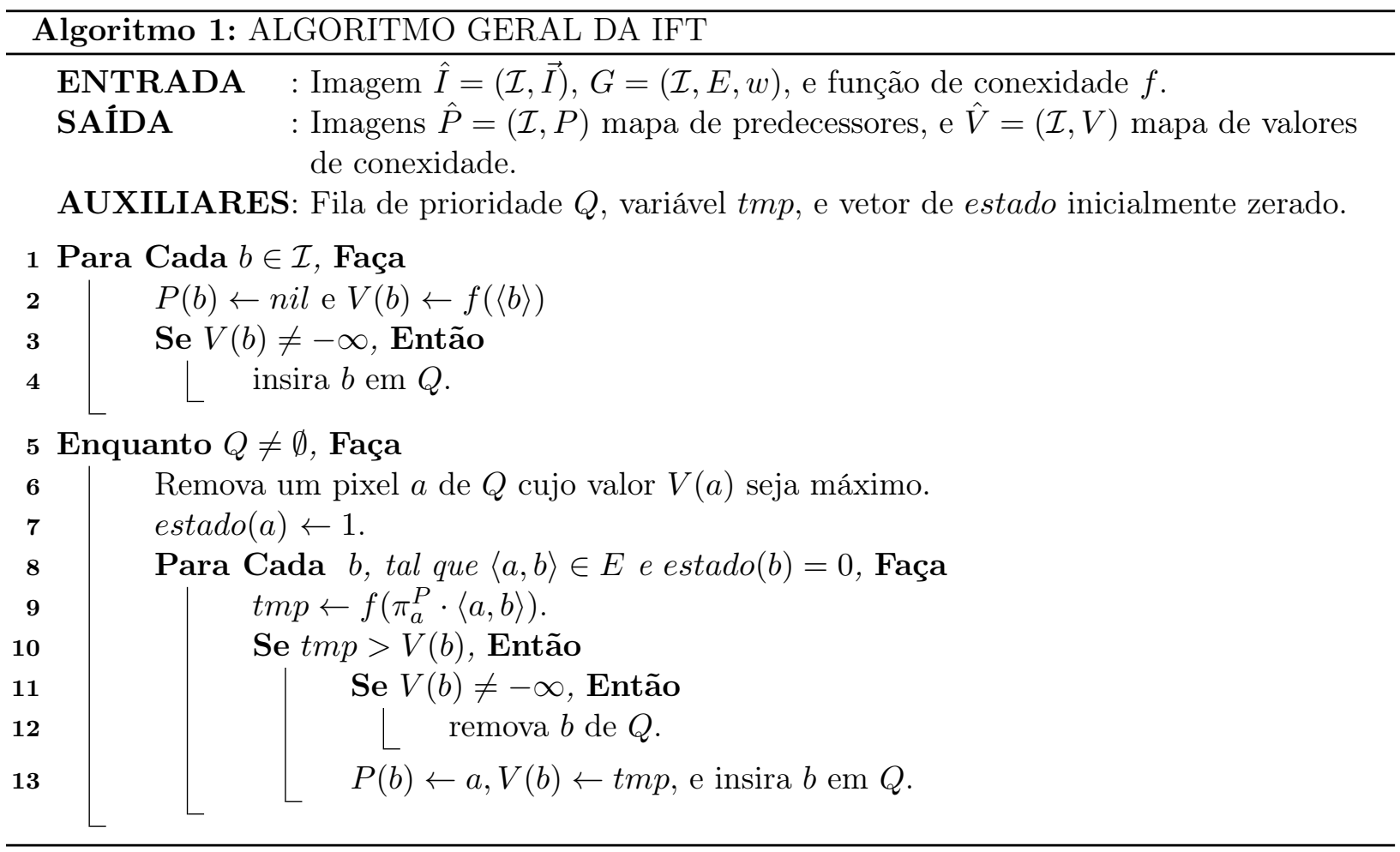

O algoritmo inicia com todos os caminhos triviais (Linhas 1 e 2). Na Linha 4, todos os candidatos a raízes (sementes) são inseridos na fila $Q$. As raízes são os máximos de $V$, primeiramente detectados na Linha 5, pois, ao remover qualquer nó $a$ de $Q$ na Linha 6 , o caminho $\pi_{a}^{P}$ é ótimo. As demais Linhas calculam e propagam caminhos melhores para os nós adjacentes. Seguindo uma ordem decrescente de conexidade e aplicando a seguinte regra de expansão: se $f\left(\pi_{a}^{P} \cdot\langle a, b\rangle\right)>f\left(\pi_{b}^{P}\right)$ (Linha 10), então $\pi_{b}^{P}$ é trocado por $\pi_{a}^{P} \cdot\langle a, b\rangle$ (Linha 13).

\subsection{Conexidade fuzzy relativa}

Nosso trabalho é baseado no método de conexidade fuzzy relativa (RFC - Relative Fuzzy Connectedness), que é um dos métodos pertencentes ao arcabouço de conexidade fuzzy (FC - Fuzzy Connectedness) [Saha e Udupa (2001); Udupa et al. (2002)]. A principal ideia do método RFC no processo de segmentação de imagem consiste na seleção dos objetos de interesse na imagem; indicados pelo usuário, ou automaticamente por pixels referidos como sementes, que competem pelos demais pixels de acordo com a força de conexidade dos melhores caminhos a partir de cada semente. Neste processo são necessários pelo menos dois objetos.

\subsubsection{A definição original por funções de conexidade}

A seguir, mostramos a definição original da conexidade fuzzy relativa como proposta em Udupa et al. (2002) para grafos simetricamente ponderados. Considere a seguinte função de conexidade:

$$
\begin{aligned}
f_{\text {min }}^{\mathcal{S}}(\langle a\rangle) & = \begin{cases}w_{\max }+1 & \text { se } a \in \mathcal{S} \\
-\infty & \text { caso contrário }\end{cases} \\
f_{\text {min }}^{\mathcal{S}}\left(\pi_{a} \cdot\langle a, b\rangle\right) & =\min \left\{f_{\text {min }}^{\mathcal{S}}\left(\pi_{a}\right), w(a, b)\right\}
\end{aligned}
$$


onde $w_{\max }=\max _{\langle a, b\rangle \in E} w(a, b)$. Dois mapas de conexidade são calculados usando duas execuções da transformada imagem-floresta [Falcão et al. (2004)]:

$$
\begin{aligned}
& V_{o}(a)=\max _{\pi_{a} \in \Pi(G, a)}\left\{f_{\text {min }}^{\mathcal{S}_{o}}\left(\pi_{a}\right)\right\}, \\
& V_{b}(a)=\max _{\pi_{a} \in \Pi(G, a)}\left\{f_{\text {min }}^{\mathcal{S}_{b}}\left(\pi_{a}\right)\right\},
\end{aligned}
$$

onde $\Pi(G, a)$ é o conjunto de todos os caminhos possíveis no grafo $G$ com término no nó " $a$ ". A segmentação $A_{R F C}\left(\mathcal{S}_{o}, \mathcal{S}_{b}\right)$ do método RFC é obtida pela comparação dos dois mapas de conexidade $V_{o}$ e $V_{b}$, tal que cada pixel $a \in \mathcal{V}$ é rotulado como pertencendo ao objeto somente se $V_{o}(a)>V_{b}(a)$ (Figura 3.3).

$$
A_{R F C}\left(\mathcal{S}_{o}, \mathcal{S}_{b}\right)=\chi_{O}: O=\left\{a \in \mathcal{V}: V_{o}(a)>V_{b}(a)\right\}
$$

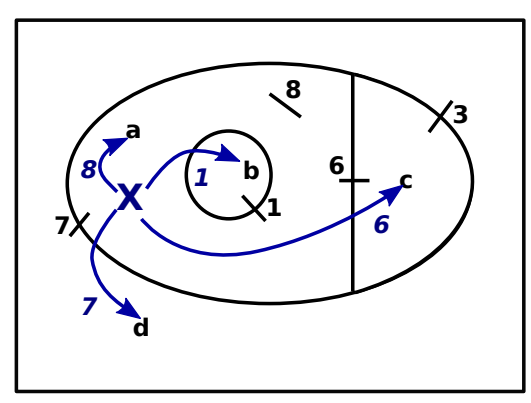

(a)

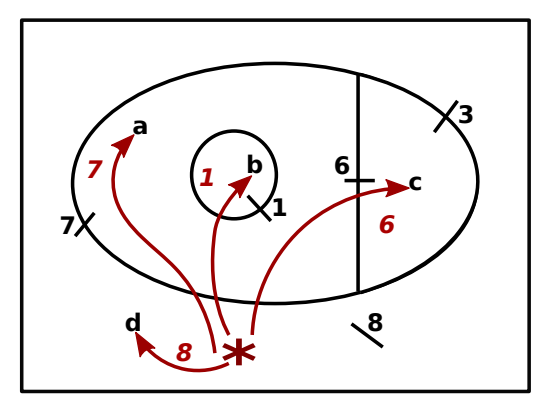

(b)

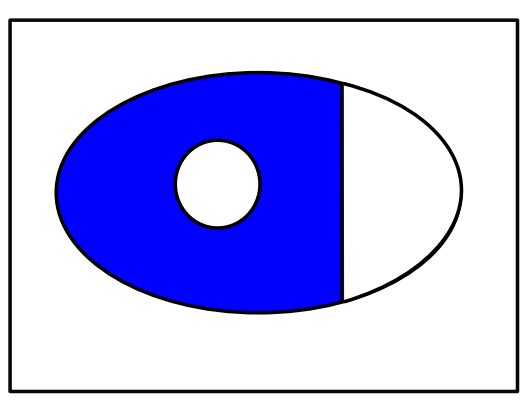

(c)

Figura 3.3: Exemplo do RFC seguindo a definição baseada em caminhos. (a) A semente de objeto $\times$, onde $f_{\min }^{\mathcal{S}_{o}}\left(\pi_{\times \rightsquigarrow a}\right)=8, f_{\min }^{\mathcal{S}_{o}}\left(\pi_{\times \rightsquigarrow b}\right)=1, f_{\min }^{\mathcal{S}_{o}}\left(\pi_{\times \rightsquigarrow c}\right)=6$ e $f_{\min }^{\mathcal{S}_{o}}\left(\pi_{\times \rightsquigarrow d}\right)=7$. (b) A semente de fundo $\star$, onde $f_{\min }^{\mathcal{S}_{b}}\left(\pi_{\star \rightsquigarrow d}\right)=8, f_{\min }^{\mathcal{S}_{b}}\left(\pi_{\star \rightsquigarrow b}\right)=1, f_{\text {min }}^{\mathcal{S}_{b}}\left(\pi_{\star \rightsquigarrow c}\right)=6$ e $f_{\min }^{\mathcal{S}_{b}}\left(\pi_{\star \rightsquigarrow a}\right)=7$. (c) Resultado de segmentação via RFC mostrada em azul escuro.

\subsubsection{Conexidade fuzzy relativa como $\varepsilon_{\infty}$-otimizador}

O método RFC também pode ser visto como um corte ótimo no grafo de acordo com uma função objetivo apropriada de corte em grafo, conforme discutido em Ciesielski e Udupa (2011); Ciesielski et al. (2012a). Para $q \in[1, \infty)$ considere o funcional de energia $\varepsilon_{q}: \tilde{\mathcal{X}} \rightarrow[0, \infty)$, onde, para todo $x \in \tilde{\mathcal{X}}, \varepsilon_{q}(x)$ é definido como a $q$-norma do funcional $F_{x}: E \rightarrow \mathbb{R}$, dado pela fórmula $F_{x}(a, b)=w(a, b)|x(a)-x(b)|$ para $\langle a, b\rangle \in E$.

Isto é, $\varepsilon_{q}(x)=\left\|F_{x}\right\|_{q}=\sqrt[q]{\sum_{\langle a, b\rangle \in E}(w(a, b)|x(a)-x(b)|)^{q}}$, para $q<\infty$.

Observe que $\lim _{q \rightarrow \infty} \varepsilon_{q}(x)=\varepsilon_{\infty}(x)$, dado que $q$-normas convergem, conforme $q \rightarrow \infty$, para a $\infty$-norma.

$$
\varepsilon_{\infty}(x)=\left\|F_{x}\right\|_{\infty}=\max _{\langle c, d\rangle \in E} w(c, d)|x(c)-x(d)|
$$

Restringindo a análise apenas para soluções binárias $x=\chi_{P} \in \mathcal{X}$, nós temos:

$$
\varepsilon_{\infty}(x)=\left\|F_{x}\right\|_{\infty}=\max _{\langle c, d\rangle \in E} w(c, d)|x(c)-x(d)|=\max _{\langle a, b\rangle \in \mathcal{C}(x)} w(a, b),
$$

onde $\mathcal{C}(x)=\{\langle a, b\rangle \in E: x(a) \neq x(b)\}$ é um conjunto de arestas de corte. Seja $\varepsilon_{\infty \downarrow}$ o mínimo da energia $\varepsilon_{\infty}(x)$ sobre todos os objetos permitidos $x \in \mathcal{X}\left(\mathcal{S}_{o}, \mathcal{S}_{b}\right)$, isto é, $\varepsilon_{\infty \downarrow}=\min \left\{\varepsilon_{\infty}(x): x \in\right.$ $\left.\mathcal{X}\left(\mathcal{S}_{o}, \mathcal{S}_{b}\right)\right\}$. Qualquer elemento de $\mathcal{X}_{\infty}\left(\mathcal{S}_{o}, \mathcal{S}_{b}\right)=\left\{x \in \mathcal{X}\left(\mathcal{S}_{o}, \mathcal{S}_{b}\right): \varepsilon_{\infty}(x)=\varepsilon_{\infty \downarrow}\right\}$ será referido como uma solução de energia ótima de $\varepsilon_{\infty}$ em $\mathcal{X}\left(\mathcal{S}_{o}, \mathcal{S}_{b}\right)$. Qualquer algoritmo $A$ que, dado um grafo e 
conjuntos de sementes $\mathcal{S}_{o}$ e $\mathcal{S}_{b}$, retorna um objeto, denotado por $A\left(\mathcal{S}_{o}, \mathcal{S}_{b}\right)$, de $\mathcal{X}_{\infty}\left(\mathcal{S}_{o}, \mathcal{S}_{b}\right)$ será referido como um algoritmo de $\varepsilon_{\infty}$-minimização. RFC é um algoritmo de $\varepsilon_{\infty}$-minimização, isto é, $A_{R F C}\left(\mathcal{S}_{o}, \mathcal{S}_{b}\right) \in \mathcal{X}_{\infty}\left(\mathcal{S}_{o}, \mathcal{S}_{b}\right)$ [Ciesielski et al. (2013)]. No caso de uma única semente $s_{1}$ (Figura 3.4), temos a seguinte definição alternativa de RFC baseada em cortes no grafo

$$
A_{R F C}\left(\left\{s_{1}\right\}, \mathcal{S}_{b}\right)=\chi_{O} \in \mathcal{X}_{\infty}\left(\left\{s_{1}\right\}, \mathcal{S}_{b}\right):|O|=\min \left\{|P|: \chi_{P} \in \mathcal{X}_{\infty}\left(\left\{s_{1}\right\}, \mathcal{S}_{b}\right)\right\}
$$

O caso de várias sementes internas é então tratado usando a seguinte equação:

$$
A_{R F C}\left(\mathcal{S}_{o}, \mathcal{S}_{b}\right)=\chi_{O}: O=\left[\bigcup_{s_{i} \in \mathcal{S}_{o}} P: \chi_{P}=A_{R F C}\left(\left\{s_{i}\right\}, \mathcal{S}_{b}\right)\right]
$$

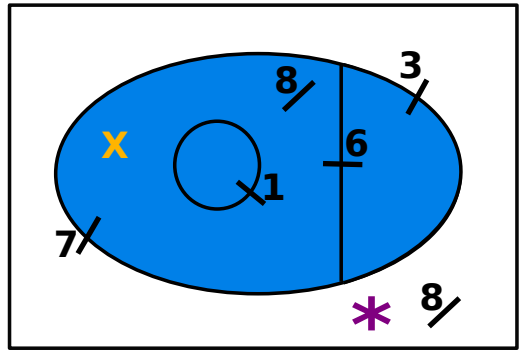

(a)

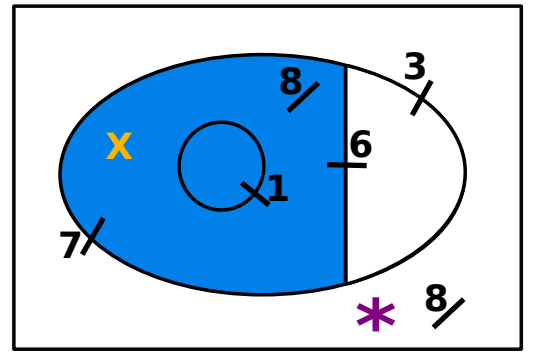

(b)

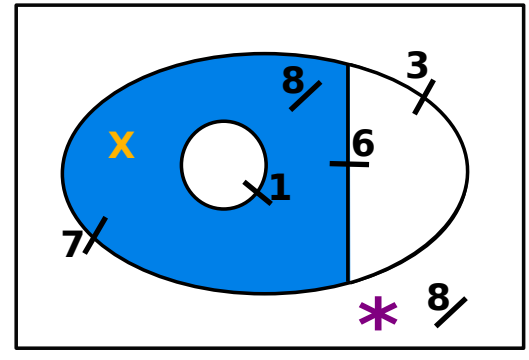

(c)

Figura 3.4: As três soluções acima $(\mathbf{a}, \mathbf{b}, \mathbf{c})$ tem energia ótima $\varepsilon_{\infty}(x)=7$, mas apenas $(\mathbf{c})$ corresponde a uma solução RFC válida de acordo com a Equação 3.6.

As vantagens da segmentação via RFC correspondem a baixo custo computacional, baixa taxa de falsos positivos e a robustez em relação a posição das sementes conforme demonstrado no trabalho [Ciesielski et al. (2007)].

A principal desvantagem é que o método pode produzir bordas com formas irregulares.

\subsection{Transformada imagem-floresta com orientação}

Um grafo ponderado dirigido é calculado, onde $w(a, b)$ é uma combinação de uma medida de similaridade regular não direcionada $\delta(a, b)$, multiplicada por um fator de orientação, do seguinte modo:

$$
w(a, b)= \begin{cases}\delta(a, b) \times(1-\alpha) & \text { se } I(a)>I(b) \\ \delta(a, b) \times(1+\alpha) & \text { se } I(a)<I(b) \\ \delta(a, b) & \text { caso contrário }\end{cases}
$$

Vários procedimentos diferentes podem ser adotados para $\delta(a, b)$, tais como o complemento do valor absoluto da diferença das intensidades da imagem (isto é, $\delta(a, b)=K-|I(a)-I(b)|)$, ou algumas funções de afinidade discutidas em Ciesielski e Udupa (2010a,b). Note que temos um grafo ponderado dirigido $(w(a, b) \neq w(b, a))$ quando $\alpha>0$ (ver exemplo da Figura 3.5).

A transformada imagem-floresta orientada (OIFT) é construída sobre o arcabouço da IFT considerando a seguinte função de caminho

$$
f_{O I F T}^{S_{1}, S_{2}}(\langle a\rangle)= \begin{cases}\left(w_{\max }+1\right) \times 2 & \text { se } a \in S_{1} \cup S_{2} \\ -\infty & \text { caso contrário }\end{cases}
$$




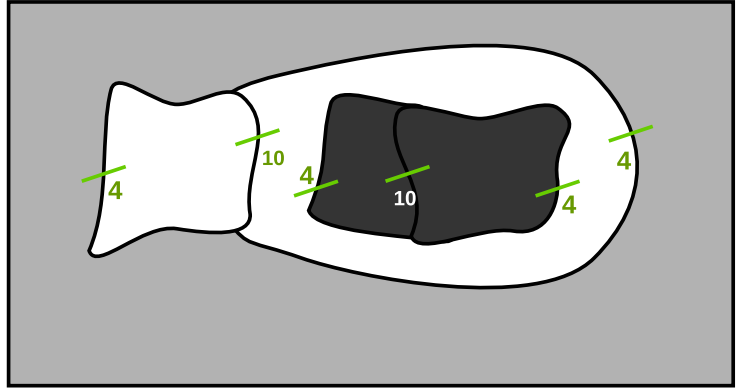

(a)

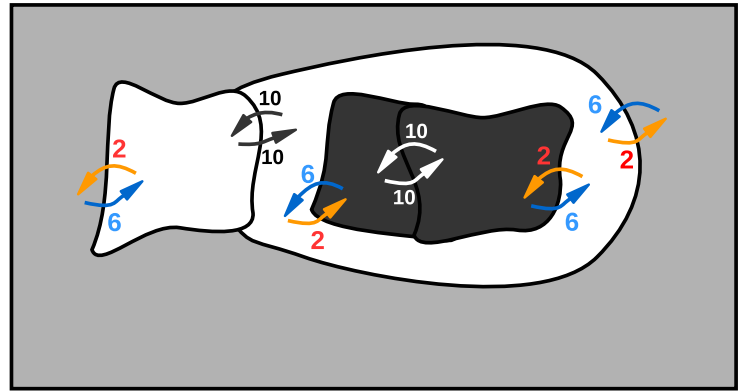

(b)

Figura 3.5: Exemplo de cálculo do fator de orientação, com valores w(a,b) (Equação 3.8). (a) Grafo não direcionado de uma imagem digital com valores $\delta(a, b)$ indicados na figura. (b) Grafo ponderado dirigido com fator de orientação $\alpha=0.5$

$$
f_{O I F T}^{S_{1}, S_{2}}\left(\pi_{x \rightsquigarrow a} \cdot\langle a, b\rangle\right)= \begin{cases}\min \left\{f_{O I F T}^{S_{1}, S_{2}}\left(\pi_{x \rightsquigarrow a}\right), 2 \cdot w(b, a)\right\} & \text { se } x \in S_{1} \\ \min \left\{f_{O I F T}^{S_{1}, S_{2}}\left(\pi_{x \rightsquigarrow a}\right), 2 \cdot w(a, b)+1\right\} & \text { caso contrário }\end{cases}
$$

OIFT possui duas versões: $A_{O I F T}^{\text {in }}\left(S_{o}, S_{b}\right)$ que favorece transições de pixels escuros para claros, e $A_{O I F T}^{\text {out }}\left(S_{o}, S_{b}\right)$ que tem a orientação oposta. $A_{O I F T}^{\text {in }}\left(S_{o}, S_{b}\right)$ (Figura 3.6a) é obtido pelo cálculo de uma IFT com função de conexidade $f_{O I F T}^{S_{o}, S_{b}}$, e, tomando como pixels do objeto o conjunto dos pixels conquistados por caminhos enraizados em $S_{o}$. $A_{O I F T}^{\text {out }}\left(S_{o}, S_{b}\right)$ (Figura 3.6b) é calculado de forma semelhante, mas utilizando $f_{O I F T}^{S_{b}, S_{o}}$.

Uma coisa importante a se notar é que a função $f_{O I F T}^{S_{1}, S_{2}}$ é uma função de conexidade nãosuave como mostrado em Miranda e Mansilla (2014). Quando a função de caminho não é suave, a IFT ainda retornará uma floresta de espalhamento, mas os caminhos calculados podem não ser ótimos [Falcão et al. (2004)]. No entanto, a otimalidade da OIFT ainda é garantida do ponto de vista de um critério de energia de corte em grafos [Mansilla e Miranda (2013a); Miranda e Mansilla (2014)].

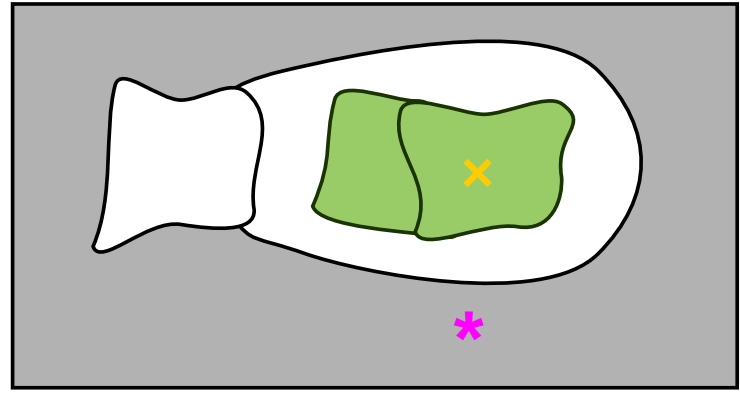

(a) $A_{O I F T}^{\text {in }}\left(\mathcal{S}_{o}, \mathcal{S}_{b}\right)$ com orientação interna

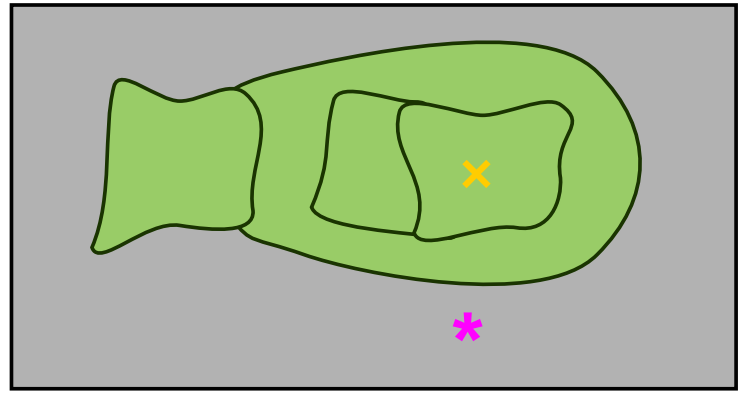

(b) $A_{O I F T}^{\text {out }}\left(\mathcal{S}_{o}, \mathcal{S}_{b}\right)$ com orientação externa

Figura 3.6: Exemplo do resultado de segmentação com o algoritmo $\operatorname{OIFT}$. (a) Algoritmo $A_{O I F T}^{\text {in }}\left(\mathcal{S}_{o}, \mathcal{S}_{b}\right)$ e (b) $A_{O I F T}^{\text {out }}\left(\mathcal{S}_{o}, \mathcal{S}_{b}\right)$.

\subsection{Método de segmentação de corte em grafo}

O algoritmo de corte em grafo (GC - Graph Cut) [Boykov e Funka-Lea (2006)] utilizado na abordagem híbrida proposta nesse trabalho é o de corte mínimo/fluxo máximo em grafos direcionados. A seguir será introduzido o problema do fluxo máximo. 


\subsubsection{Problema do fluxo máximo}

Podemos interpretar um grafo direcionado como uma "rede de fluxo" e usá-lo para resolver problemas sobre os fluxos de materiais. Imaginemos um material fluindo através de um sistema a partir de uma fonte, onde o material é produzido, para um sorvedouro, onde é consumido. A fonte produz o material em alguma taxa constante, e o sorvedouro consome o material com a mesma velocidade. No problema de fluxo máximo, queremos calcular a maior taxa de transmissão de material a partir da fonte para o coletor sem violar quaisquer restrições de capacidade. Figura 3.7 mostra um exemplo de fluxo.

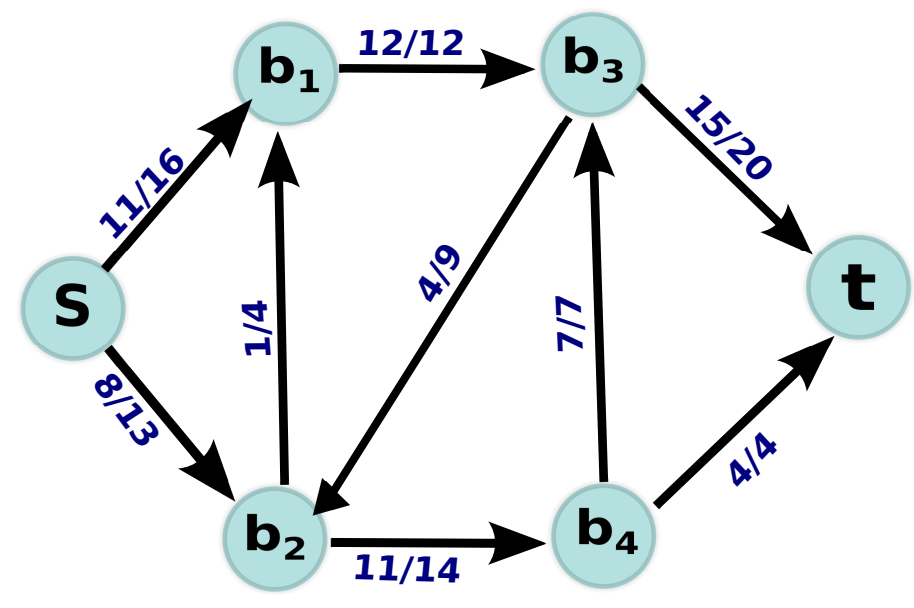

Figura 3.7: O fluxo $f$ com valor $|f|=19$. Cada arco $\langle a, b\rangle$ é rotulado por um par de fluxo e capacidade $f(a, b) / c(a, b)$. A notação de barra apenas separa o fluxo e a capacidade; ele não indica divisão.

\subsubsection{Fluxo no grafo}

Uma rede de fluxo $G=(\mathcal{V}, E)$ é um grafo direcionado no qual cada arco $\langle a, b\rangle \in E$ possui uma capacidade não negativa $c(a, b) \geq 0$, onde $s$ é o vértice fonte e $t$ é o vértice destino. Por conveniência, assumimos que cada vértice encontra-se em algum caminho a partir da fonte para o destino. Isto é, para cada vértice $b \in \mathcal{V}$, a rede de fluxo contém um caminho $s \rightsquigarrow b \rightsquigarrow t$. O grafo é, portanto, conexo, já que cada vértice diferente de s tem, pelo menos, um arco de entrada, $|E| \geq|\mathcal{V}|-1$.

Dado uma rede de fluxo $G$ com fonte $s$ e destino $t$, queremos encontrar um fluxo de valor máximo indo de $s$ para $t$.

A função capacidade é definida como sendo: $c: E \rightarrow \mathbb{R}$ e a função de fluxo $f: \mathcal{V} \times \mathcal{V} \rightarrow \mathbb{R}$, sendo que elas devem satisfazer as seguintes restrições:

1. Restrição de capacidade: O fluxo não viola a capacidade máxima da aresta.

$$
f(a, b) \leq c(a, b), \forall a, b \in \mathcal{V}
$$

2. Conservação de fluxo: O saldo do fluxo que entra e sai de um vértice do grafo é nulo, com exceção dos vértices $s$ (fonte) e $t$ (sorvedouro).

$$
\sum_{b \in \mathcal{V}} f(b, a)=\sum_{b \in \mathcal{V}} f(a, b), \forall a \in \mathcal{V}-\{s, t\}
$$


3. Anti-simetria: O fluxo de arestas anti-paralelas possui fluxo inverso.

$$
f(a, b)=-f(b, a), \forall a, b \in \mathcal{V}
$$

Definimos o fluxo de arcos inexistentes como nulo:

$$
f(a, b)=0, \forall\langle a, b\rangle \notin E
$$

Definimos o grafo residual $G_{f}=\left(\mathcal{V}, E_{f}, c_{f}\right)$ e a função capacidade residual $c_{f}(a, b)=c(a, b)-$ $f(a, b)$, onde $E_{f}=\left\{\langle a, b\rangle \in \mathcal{V} \times \mathcal{V}: c_{f}(a, b)>0\right\}$.

Para calcular o fluxo máximo, podemos usar o método de Ford-Fulkerson (Algoritmo 2) [T.H. Cormen e Stein (2009)].

- Começamos com fluxo inicial zero (ou seja, $f(a, b)=0$ para todos $a, b \in \mathcal{V}$ ).

- A cada iteração, aumentamos o fluxo total encontrando algum caminho (a partir da fonte $s$ até o destino $t$ ) ao longo do qual podemos empurrar mais fluxo(caminho de aumento (augmenting paths)).

- Repetimos este processo até que nenhum camiho de aumento pode ser encontrado.

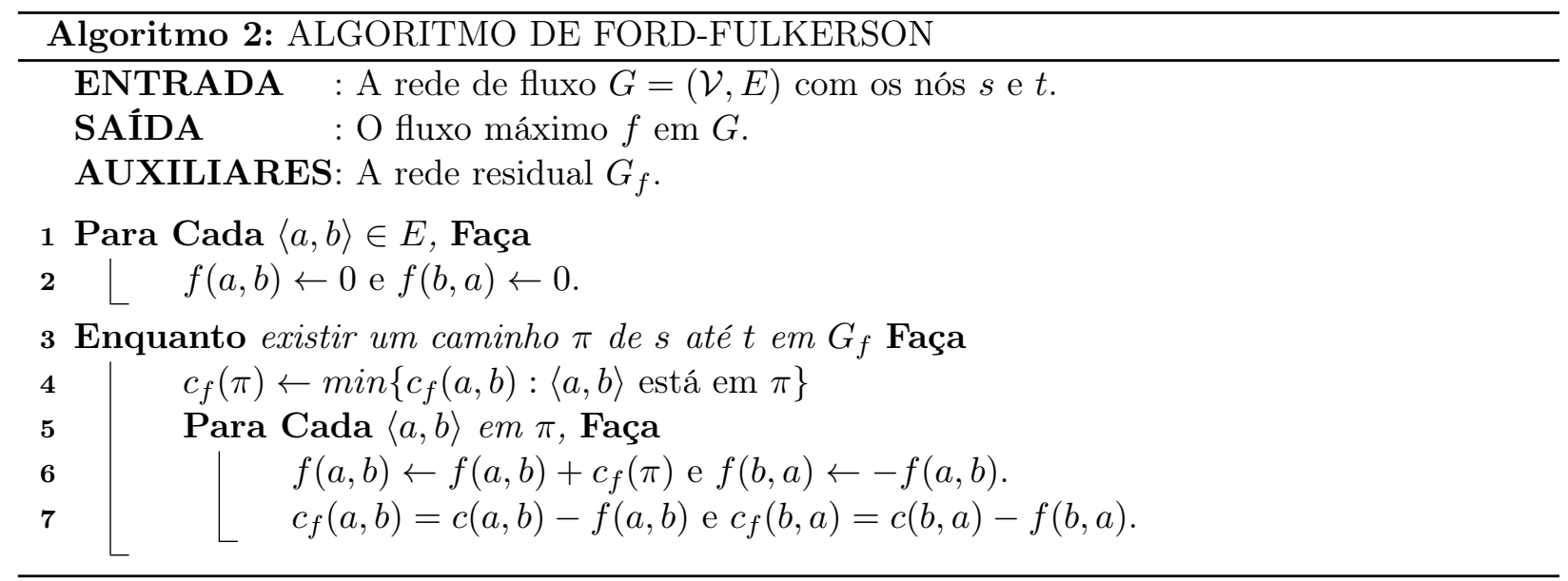

\subsubsection{Corte mínimo em Grafo}

Considere um grafo dirigido $G$, onde os pesos não negativos $w(a, b)$ de cada arco correspondem aos valores de capacidade iniciais da rede, isto é, $w(a, b)=c(a, b)$.

O corte mínimo é o subconjunto não trivial $S$ do $\mathcal{V}$ de tal modo que a soma dos arcos entre os nós em $S$ e o seu complemento é minimizada, isto é, o minimizador de

$$
\varepsilon_{1}^{\text {out }}=\operatorname{cut}(S, \mathcal{V})=\sum_{a \in S \wedge b \in \mathcal{V} \backslash S} w(a, b)
$$

Segundo o teorema do fluxo máximo/corte mínimo, o valor do fluxo máximo de $s$ para $t$ corresponde ao valor do corte mínimo separando os nós $s$ e $t$ (o valor do fluxo máximo é igual ao mínimo de $\operatorname{cut}(S, \mathcal{V})$ tal que $s \in S$ e $t \in \mathcal{V} \backslash S)$.

\subsubsection{Arcabouço de segmentação de corte em grafo}

O grafo $G=(\mathcal{V}, E, w)$ associado à imagem $\hat{I}=(\mathcal{I}, \vec{I})$, é modificado ligeiramente. Mais especificamente, o conjunto de vérties $\mathcal{V}$ é definido como $\mathcal{I} \cup\{s, t\}$, isto é, o conjunto de vértices 
da imagem $\mathcal{I}$ é ampliado por dois novos vértices adicionais $s$ e $t$ chamados nós terminais. Individualmente, $s$ é referido como fonte e $t$ como destino. O conjunto de arcos é definido como $E=E_{c} \cup\{\langle s, a\rangle: a \in \mathcal{I}\} \cup\{\langle b, t\rangle: b \in \mathcal{I}\}$, onde $E_{c}$ é um conjunto de arcos definidos por uma relação de adjacência circular em $\mathcal{I}$, conforme discutido na Seção 2.2 .

A maneira mais simples para pensar sobre os terminais é que eles servem como indicadores de sementes: $s$ para sementes $\mathcal{S}_{o} \subset \mathcal{I}$ indicando o objeto; $t$ para sementes $\mathcal{S}_{b} \subset \mathcal{I}$ indicando o fundo. Para cada arco de conexão de um terminal $r \in\{s, t\}$ com o pixel $a \in \mathcal{I}$, associar o peso: $\infty$ se $r=s$ e $a \in \mathcal{S}_{o}$ ou $r=t$ e $a \in \mathcal{S}_{b}$; e 0 caso contrário. Isto significa, que a fonte $s$ tem uma ligação infinitamente forte com qualquer semente $a \mathrm{em} \mathcal{S}_{o}$, e o mais fraco possível com qualquer outro $a \mathrm{em}$ $\mathcal{I}$. Assume-se que todos os pesos são não-negativo. Da mesma forma, para o destino $t$ e sementes $a$ de $\mathcal{S}_{b}$.

Conforme visto na Figura 1.1, o GC tem como vantagem a geração de bordas mais suaves e regulares, em comparação com métodos baseados puramente na IFT.

\subsubsection{Problema de viés de encolhimento}

O algoritmo de corte mínimo/fluxo máximo é um dos métodos mais comumente utilizados para dividir grafos. No entanto tem-se observado que este critério tem uma tendência para produzir cortes desequilibrados: Ele apresenta viés por bordas pequenas, isto é, objetos com valores baixos de perímetro (Figura 3.8).



(a)

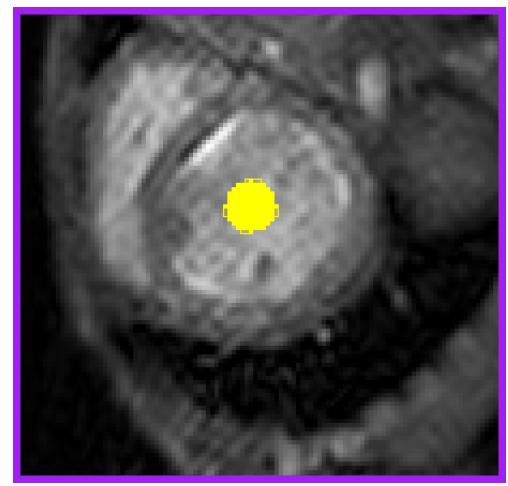

(b)



(c)

Figura 3.8: Exemplo de viés de encolhimento, onde os pixels em amarelo indicam as sementes internas e as sementes externas são indicadas em roxo. (a-b) Segmentações indesejadas são obtidas quando o número de sementes internas é insuficiente (o objeto delineado colapsa para as sementes internas), (c) resultado da segmentação com um conjunto maior de sementes internas.

Em contraste com o método RFC, o método GC tem a tendência de escolher os objetos com tamanho muito pequeno no contorno, até mesmo quando os pesos das arestas de delimitação são muito altas [Boykov e Funka-Lea (2006)], este comportamento é referido como viés de encolhimento [Vicente et al. (2008)]. Isso leva facilmente o objeto segmentado a ficar muito próximo ao conjunto de sementes do objeto $\mathcal{S}_{o}$, ou ao complemento do conjunto de sementes do fundo $\mathcal{S}_{b}$. Portanto, o objeto retornado no GC pode estar longe do desejável. Este problema tem sido abordado por muitos autores, através de modificação do método de GC. Observe que o método RFC não tem nenhum problema de encolhimento [Ciesielski et al. (2013)]. 


\section{Capítulo 4}

\section{Conexidade fuzzy relativa com orientação}

Diferentemente da conexidade fuzzy relativa (RFC - Relative Fuzzy Connectedness), que possui duas definições equivalentes (por funções de conexidade e $\varepsilon_{\infty}$-otimizador), as definições do método de conexidade fuzzy relativa com orientação (ORFC - Oriented Relative Fuzzy Connectedness) baseada em caminhos e baseada em cortes no dígrafo conduzem a resultados diferentes (Figuras 4.1ab). Os diferentes algoritmos obtidos serão denominados como $A_{O R F C}^{i n, \rightsquigarrow}$ e $A_{O R F C}^{\text {out, } \leadsto \text { para a definição }}$ baseada em caminho; e $A_{O R F C}^{i n, \diamond<}$ e $A_{O R F C}^{\text {out,ox }}$ para a definição baseada em corte.

\subsection{Definição da ORFC por funções de conexidade inversa}

Com base nos trabalhos anteriores Mansilla e Miranda (2013a); Miranda e Mansilla (2014), nós consideramos a seguinte nova função de conexidade em dígrafos:

$$
\begin{aligned}
f_{\min }^{\nvdash \mathcal{S}}(\langle a\rangle) & = \begin{cases}w_{\max }+1 & \text { se } a \in \mathcal{S} \\
-\infty & \text { caso contrário }\end{cases} \\
f_{\min }^{\nvdash \mathcal{S}}\left(\pi_{a} \cdot\langle a, b\rangle\right) & =\min \left\{f_{\min }^{\nvdash \mathcal{S}}\left(\pi_{a}\right), w(b, a)\right\}
\end{aligned}
$$

onde $\langle b, a\rangle$ é um arco anti-paralelo.

Observe que $f_{\min }^{\nVdash \mathcal{S}}$ é uma função suave, e por conseguinte $V_{o}^{\nVdash}$ e $V_{b}^{\nVdash}$ são mapas de conexidade que podem ser obtidos via IFT. Estes dois mapas de conexidade são gerados por meio de duas execuções da IFT com funções de conexidade anti-paralelas:

$$
\begin{aligned}
& V_{o}^{K}(a)=\max _{\pi_{a} \in \Pi(G, a)}\left\{f_{\text {min }}^{\nVdash S_{o}}\left(\pi_{a}\right)\right\} \\
& V_{b}^{K}(a)=\max _{\pi_{a} \in \Pi(G, a)}\left\{f_{\min }^{\nVdash S_{b}}\left(\pi_{a}\right)\right\}
\end{aligned}
$$

Seguindo a mesma ideia chave de Mansilla e Miranda (2013a) (isto é, considerar funções de conexidade reversas para um dos conjuntos de sementes), nós temos a seguinte definição natural para $O R F C$ : A segmentação $A_{O R F C}^{\text {out } \leadsto}\left(S_{o}, S_{b}\right)$, favorecendo transições de pixels de claro para escuro, é obtida comparando os mapas de conexidade $V_{o}(a)$ e $V_{b}^{\mathbb{K}}(a)$, de tal modo que cada pixel $a \in \mathcal{V}$ é rotulado como pertencendo ao objeto somente se $V_{o}(a)>V_{b}^{\nVdash}(a)$.

$$
A_{O R F C}^{\text {out } \leadsto}\left(S_{o}, S_{b}\right)=\chi_{O}: O=\left\{a \in \mathcal{V}: V_{o}(a)>V_{b}^{K}(a)\right\}
$$

A segmentação $A_{O R F C}^{i n, \rightsquigarrow}\left(\left\{S_{o}, S_{b}\right)\right.$, favorecendo transições de pixels de escuro para claro, é obtida comparando os mapas de conexidade $V_{o}^{\nVdash}(a)$ e $V_{b}(a)$, de tal modo que cada pixel $a \in \mathcal{V}$ é rotulado 
como pertencendo ao objeto somente se $V_{o}^{\nVdash}(a)>V_{b}(a)$.

$$
A_{O R F C}^{i n, \rightsquigarrow}\left(S_{o}, S_{b}\right)=\chi_{O}: O=\left\{a \in \mathcal{V}: V_{o}^{\nVdash}(a)>V_{b}(a)\right\}
$$

Note que embora esta versão $O R F C$ é baseada em mapas de conexidade ótimos, seus resultados práticos têm características indesejáveis, tais como a presença de regiões desconexas e altas taxas de falso-positivos, conduzindo a resultados insatisfatórios (Figura 4.1a).

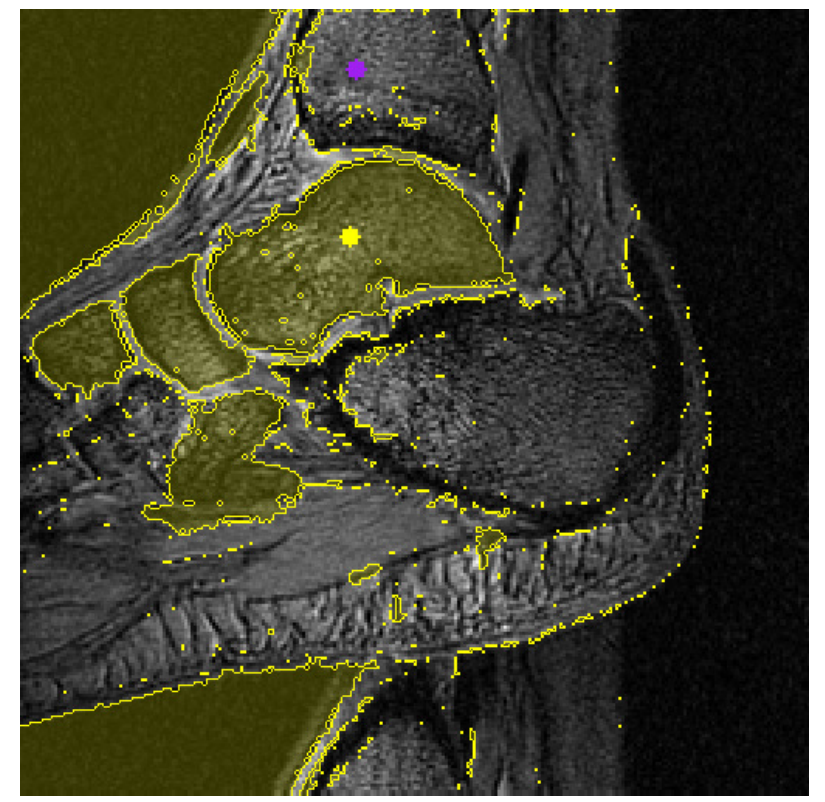

(a)

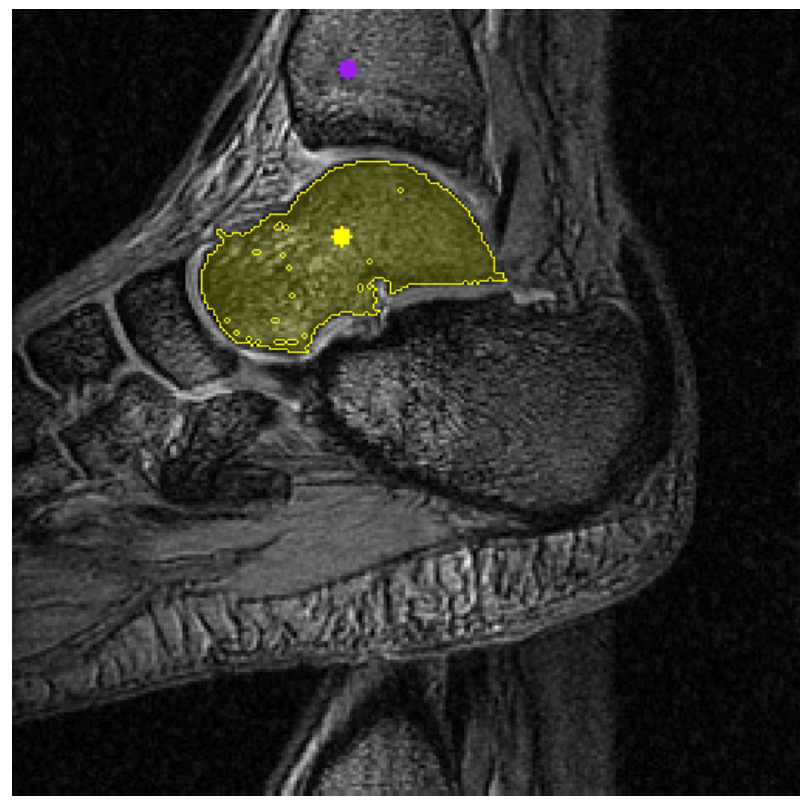

(b)

Figura 4.1: (a) ORFC por funções de conexidade inversa, com a orientação de pixels de escuro para claro $\left(A_{O R F C}^{i n, \rightsquigarrow}\right)$. (b) ORFC como corte dirigido no dígrafo $\left(A_{O R F C}^{i n, a<}\right)$.

\subsection{Definição ORFC como um corte dirigido no dígrafo}

Dado que a definição anterior de ORFC por funções de conexidade (Seção 4.1) apresenta resultados indesejados, nesta seção, apresentamos uma definição alternativa suportada por um critério de otimização de corte em grafo, a qual é motivada pelas definições da Seção 3.2.2.

Diferentemente da Seção 3.2.2, no caso de grafos dirigidos, temos dois possíveis conjuntos de cortes (Figura 4.2):

$$
\begin{gathered}
\mathcal{C}_{\text {out }}(x)=\{\langle a, b\rangle \in E: x(a)=1 \wedge x(b)=0\} \\
\mathcal{C}_{\text {in }}(x)=\{\langle a, b\rangle \in E: x(a)=0 \wedge x(b)=1\}
\end{gathered}
$$

Portanto, temos duas formulações possíveis para o funcional de energia do problema de minimização da $\varepsilon_{\infty}$.

$$
\begin{aligned}
\varepsilon_{\infty}^{\text {out }}(x) & =\max _{\langle a, b\rangle \in \mathcal{C}_{\text {out }}} w(a, b) \\
\varepsilon_{\infty}^{\text {in }}(x) & =\max _{\langle a, b\rangle \in \mathcal{C}_{\text {in }}} w(a, b)
\end{aligned}
$$

Seja $\varepsilon_{\infty \downarrow}^{\text {out }}$ o valor mínimo da energia $\varepsilon_{\infty}^{\text {out }}(x)$, isto é:

$$
\varepsilon_{\infty \downarrow}^{\text {out }}=\min \left\{\varepsilon_{\infty}^{\text {out }}(x): x \in \mathcal{X}\left(\mathcal{S}_{o}, \mathcal{S}_{b}\right)\right\}
$$




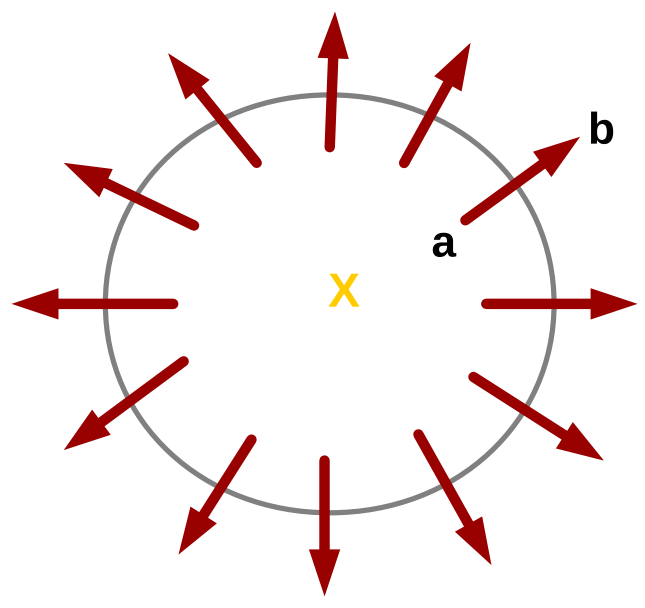

$\mathcal{C}_{\text {out }}$

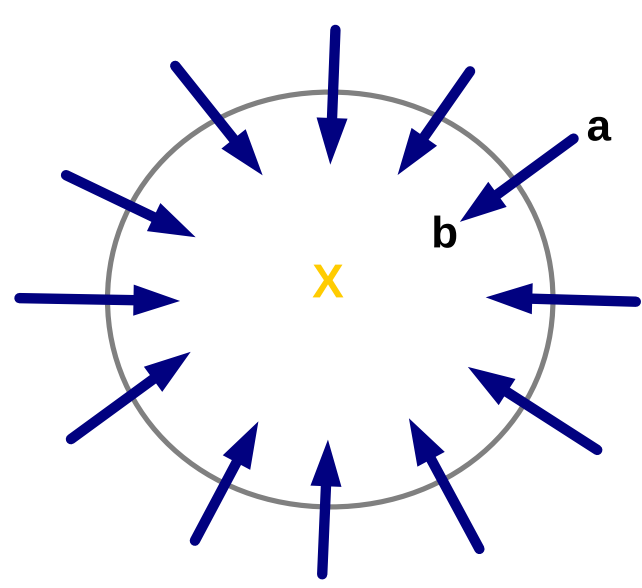

$\mathcal{C}_{\text {in }}$

Figura 4.2: Os cortes externos $\left(\mathcal{C}_{\text {out }}\right)$ e internos $\left(\mathcal{C}_{\text {in }}\right)$ para um objeto candidato mostrando os arcos de saída e de entrada.

Da mesma forma, para $\varepsilon_{\infty}^{i n}(x)$, nós temos:

$$
\varepsilon_{\infty \downarrow}^{i n}=\min \left\{\varepsilon_{\infty}^{i n}(x): x \in \mathcal{X}\left(\mathcal{S}_{o}, \mathcal{S}_{b}\right)\right\}
$$

Portanto, temos os seguintes conjuntos de soluções:

$$
\begin{gathered}
\mathcal{X}_{\infty}^{\text {out }}\left(\mathcal{S}_{o}, \mathcal{S}_{b}\right)=\left\{x \in \mathcal{X}\left(\mathcal{S}_{o}, \mathcal{S}_{b}\right): \varepsilon_{\infty}^{\text {out }}(x)=\varepsilon_{\infty \downarrow}^{\text {out }}\right\} \\
\mathcal{X}_{\infty}^{\text {in }}\left(\mathcal{S}_{o}, \mathcal{S}_{b}\right)=\left\{x \in \mathcal{X}\left(\mathcal{S}_{o}, \mathcal{S}_{b}\right): \varepsilon_{\infty}^{\text {in }}(x)=\varepsilon_{\infty \downarrow}^{\text {in }}\right\}
\end{gathered}
$$

Os algoritmos da ORFC em dígrafos têm as seguintes definições baseadas em corte no grafo: Para o corte externo "out" com uma única semente interna $s_{1}$,

$$
A_{O R F C}^{\text {out, }<}\left(\left\{s_{1}\right\}, \mathcal{S}_{b}\right)=\chi_{O} \in \mathcal{X}_{\infty}^{\text {out }}\left(\left\{s_{1}\right\}, \mathcal{S}_{b}\right):|O|=\min \left\{|P|: \chi_{P} \in \mathcal{X}_{\infty}^{\text {out }}\left(\left\{s_{1}\right\}, \mathcal{S}_{b}\right)\right\}
$$

e no caso de múltiplas sementes internas,

$$
A_{O R F C}^{\text {out,o< }}\left(\mathcal{S}_{o}, \mathcal{S}_{b}\right)=\chi_{O}: O=\left[\bigcup_{s_{i} \in \mathcal{S}_{o}} P: \chi_{P}=A_{O R F C}^{\text {out,o< }}\left(\left\{s_{i}\right\}, \mathcal{S}_{b}\right)\right]
$$

Para o corte interno "in" com uma única semente interna $s_{1}$,

$$
A_{O R F C}^{i n, \text {, }}\left(\left\{s_{1}\right\}, \mathcal{S}_{b}\right)=\chi_{O} \in \mathcal{X}_{\infty}^{i n}\left(\left\{s_{1}\right\}, \mathcal{S}_{b}\right):|O|=\min \left\{|P|: \chi_{P} \in \mathcal{X}_{\infty}^{i n}\left(\left\{s_{1}\right\}, \mathcal{S}_{b}\right)\right\}
$$

e no caso de múltiplas sementes internas,

$$
A_{O R F C}^{i n, a<}\left(\mathcal{S}_{o}, \mathcal{S}_{b}\right)=\chi_{O}: O=\left[\bigcup_{s_{i} \in \mathcal{S}_{o}} P: \chi_{P}=A_{O R F C}^{i n, a<}\left(\left\{s_{i}\right\}, \mathcal{S}_{b}\right)\right]
$$

\subsection{Algoritmo ORFC baseado em corte no grafo}

A fim de mostrar os algoritmos propostos, temos a seguinte definição: 
Definição 1 (Componente Conexo Dirigido - (DCC)). Para um dado vértice $x$ de um dígrafo $G$, o componente conexo dirigido com ponto base $x$ é o conjunto, denotado por $D C C_{G}(x)$ (Figura 4.3), de todos os sucessores de $x$ em $G$ (isto é, todos os nós que estão ao alcance do vértice $x$ por algum caminho).

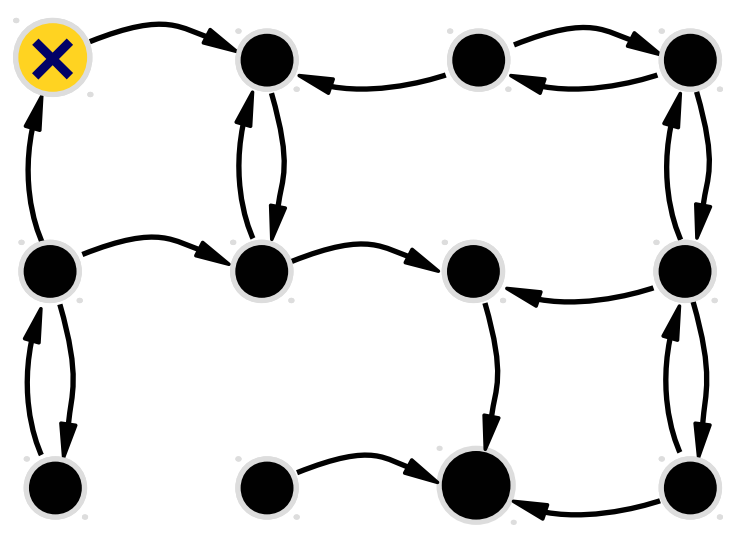

(a)

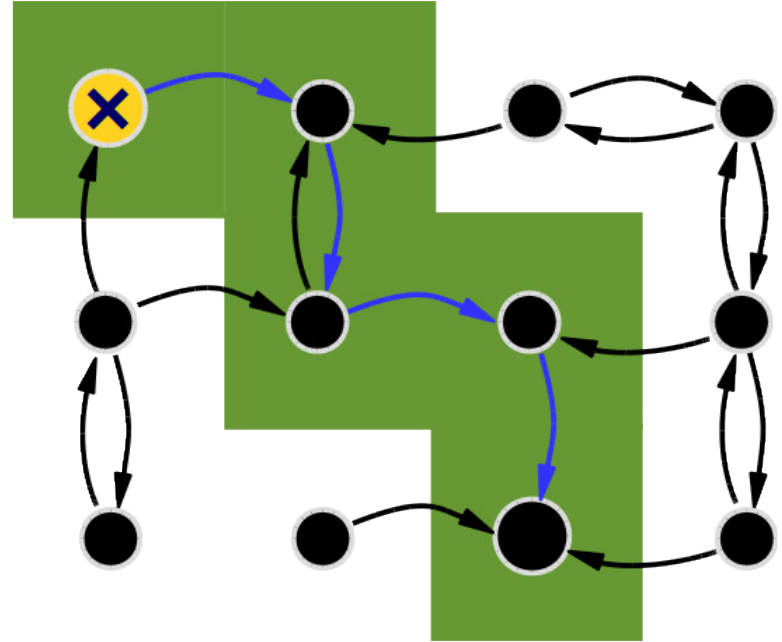

(b)

Figura 4.3: Exemplo de componente conexo dirigido (DCC). (a) Grafo ponderado dirigido $G=(\mathcal{V}, E, w)$, (b) componente conexo dirigido no grafo $D C C_{G}(x)$.

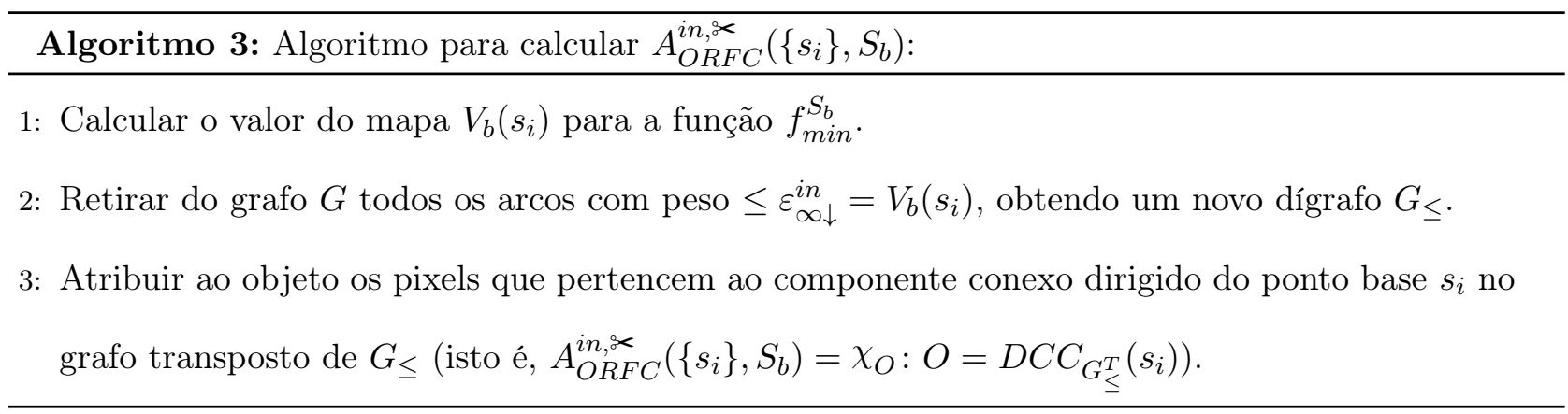

Exemplos da execução do Algoritmo 3 são apresentados nas Figura 4.4 e Figura 4.5.

Algoritmo 4: Algoritmo para calcular $A_{O R F C}^{\text {out,oe }}\left(\left\{s_{i}\right\}, S_{b}\right)$ :

1: Calcular o valor do mapa $V_{b}^{\nVdash}\left(s_{i}\right)$ para a função $f_{m i n}^{\nVdash S_{b}}$.

2: Retirar do grafo $G$ todos os arcos com peso $\leq \varepsilon_{\infty \downarrow}^{\text {out }}=V_{b}^{K}\left(s_{i}\right)$, obtendo um novo dígrafo $G_{\leq}$.

3: Atribuir ao objeto os pixels que pertencem ao componente conexo dirigido do ponto base $s_{i}$ no grafo $G_{\leq}$(isto é, $\left.A_{O R F C}^{\text {out, iæ }}\left(\left\{s_{i}\right\}, S_{b}\right)=\chi_{O}: O=D C C_{G_{\leq}}\left(s_{i}\right)\right)$.

Um exemplo da execução do Algoritmo 4 é apresentado na Figura 4.6. 


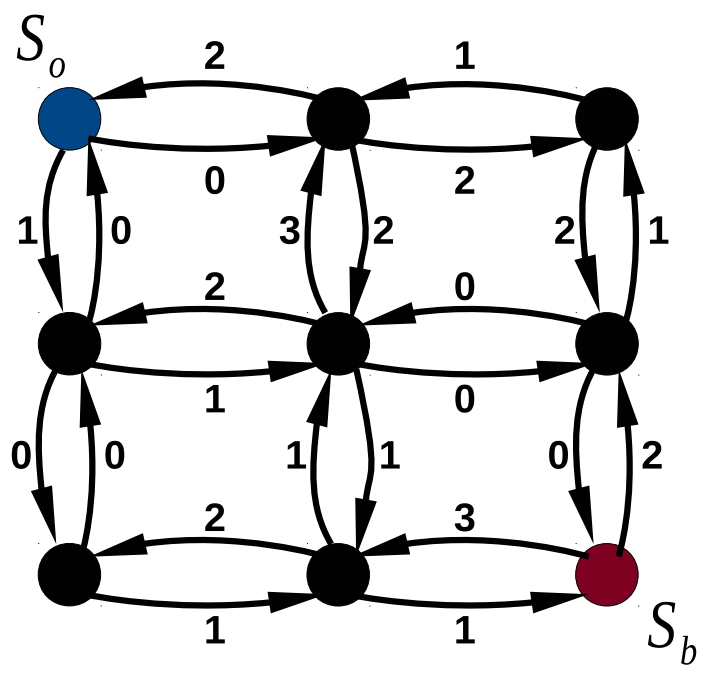

(a) $G=(\mathcal{V}, E, w)$

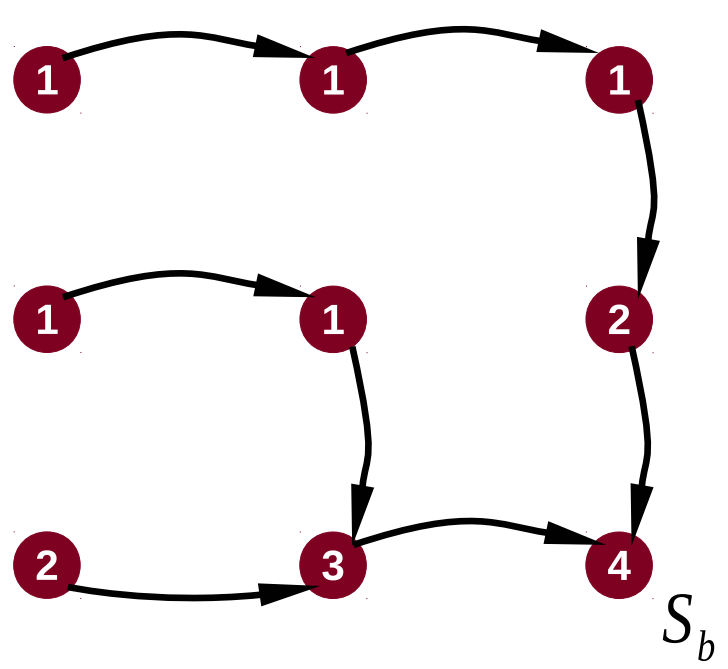

(c) $V_{b}\left(s_{i}\right)=1$

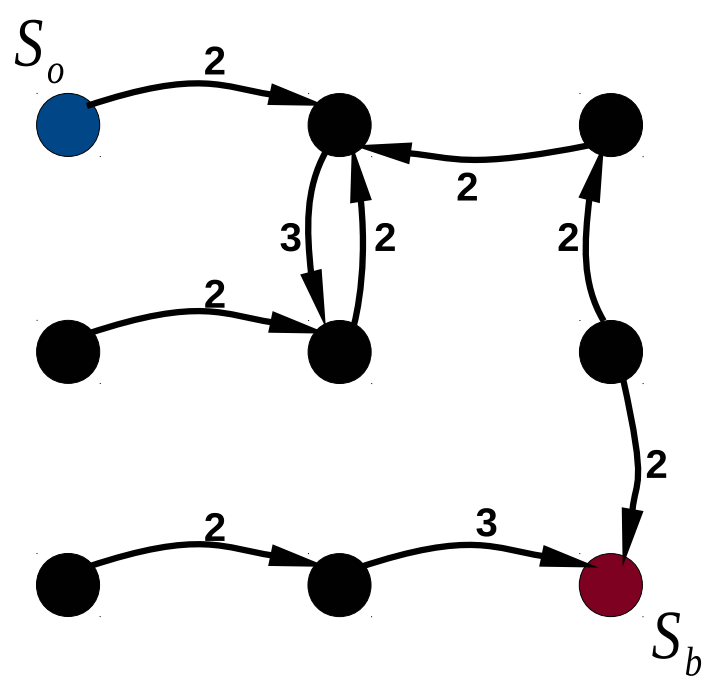

(e) $G_{\leq}^{T}$ $\infty$

$\infty$

$\infty$

$\infty$

$\infty$

$\infty$

$\infty$

$\infty$

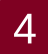
$S_{b}$ (b) Inicialização $f_{\text {min }}^{\mathcal{S}_{b}}$

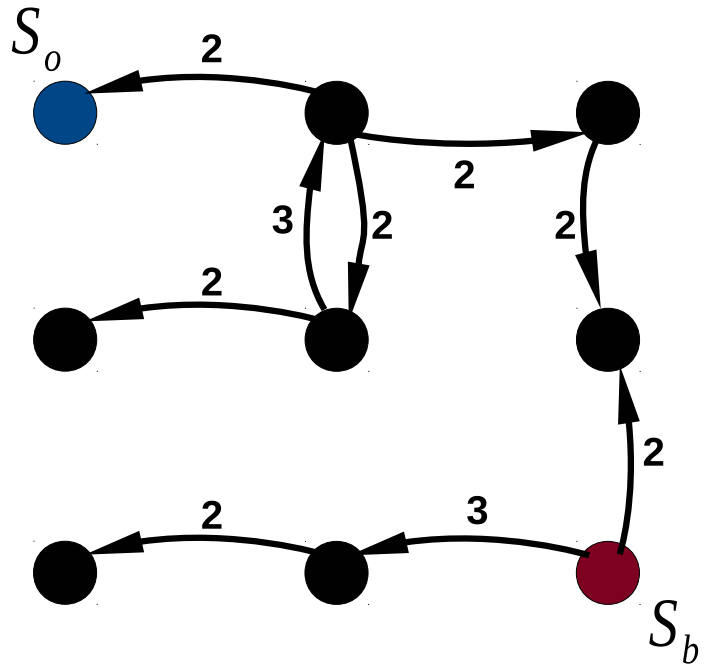

(d) $G_{\leq}$

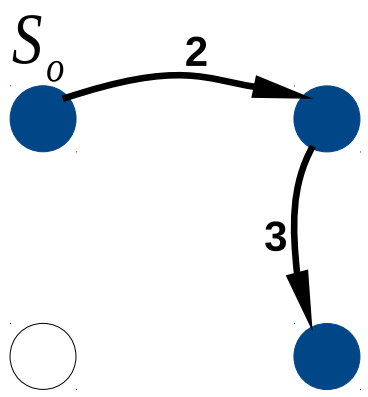

$S_{b}$

(f) Resultado

Figura 4.4: Algoritmo $A_{O R F C}^{\text {in,s< }}\left(\mathcal{S}_{o}=\left\{s_{i}\right\}, \mathcal{S}_{b}\right)$. (a) Imagem como dígrafo. (b) Inicialização da IFT com semente de fundo $\mathcal{S}_{b}$ para calcular o valor da conexidade $V_{b}\left(s_{i}\right)$ usando a função de conexidade $f_{\text {min }}^{\mathcal{S}_{b}}$. (c) Resultado da etapa 1: $O$ valor $V_{b}\left(s_{i}\right)=1$ é calculado mediante a IFT. (d) Etapa 2: O grafo $G_{<}$. (e,f) Etapa 3: a transposta do grafo $G_{\leq}$e finalmente, os pixels do objeto do DCC. 


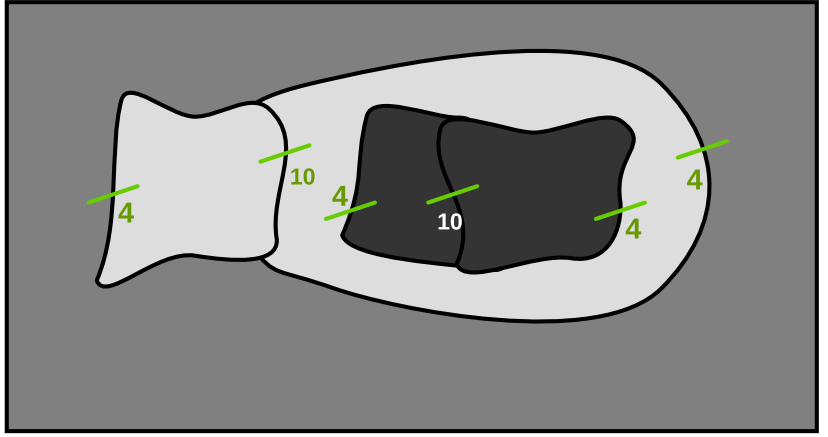

(a) $G=(\mathcal{V}, E, w)$

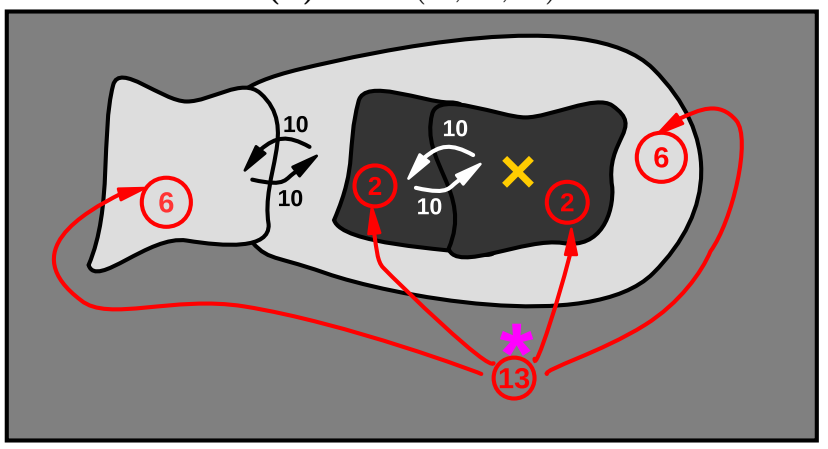

(c) $V_{b}\left(s_{i}\right)=2$

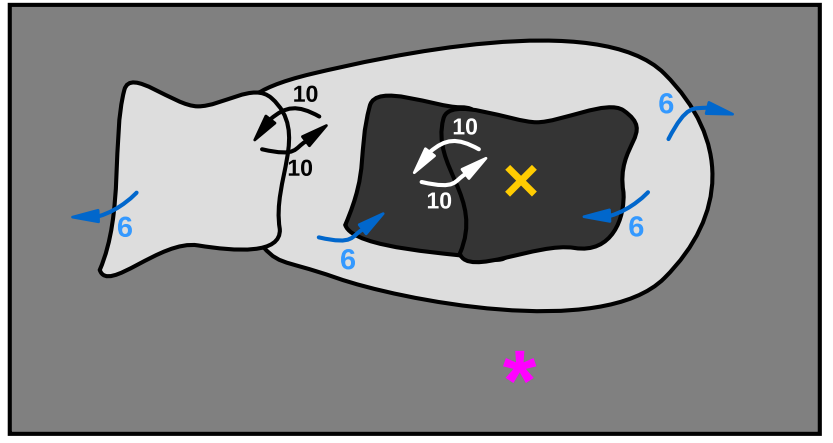

(e) $G_{\leq}^{T}$

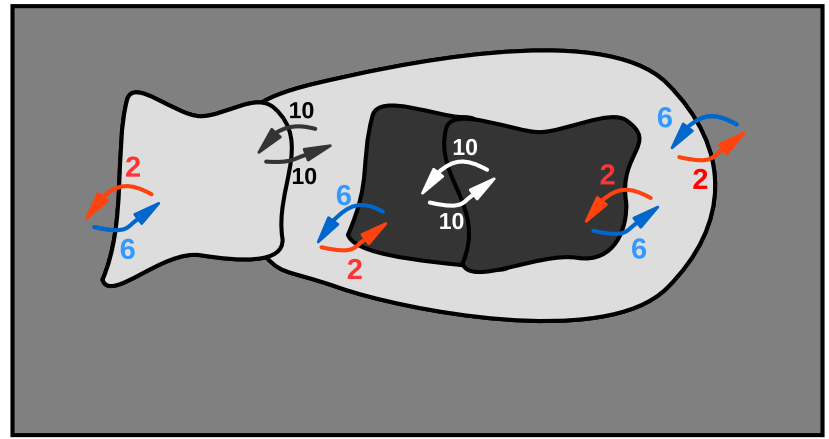

(b) $\alpha=0.5$

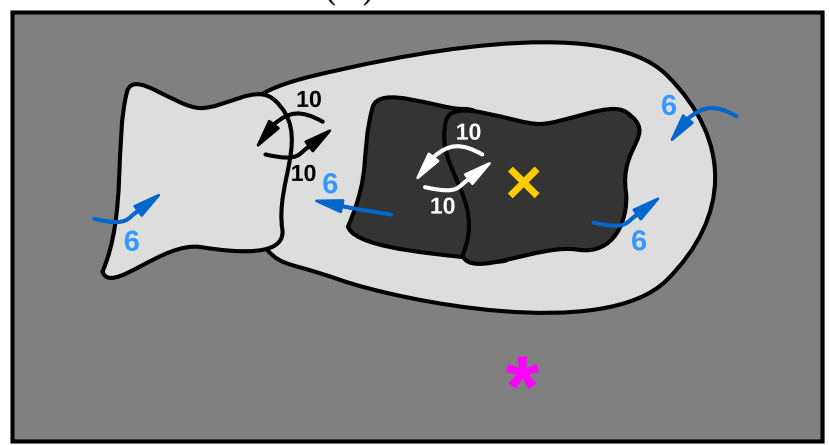

(d) $G_{<}$



(f) Resultado

Figura 4.5: Algoritmo $A_{O R F C}^{\text {in, } \odot \times}\left(\left\{s_{i}\right\}, \mathcal{S}_{b}\right)$. Grafo a partir de uma imagem com semente de objeto $\times$ e semente de fundo *, (a) grafo não dirigido, (b) dígrado com fator de orientação $\alpha=0.5$ (c) resutado do cálculo do mapa de conexidade $V_{b}\left(s_{i}\right)=2$ para uma função de conexidade $f_{\min }^{\mathcal{S}_{b}}$, (d) obtenção do grafo residual $G \leq$, (e) o grafo transposto $G_{\leq}^{T}$, (f) objeto composto pelos pixels que pertencem ao $D C C\left(s_{i}\right)$. 


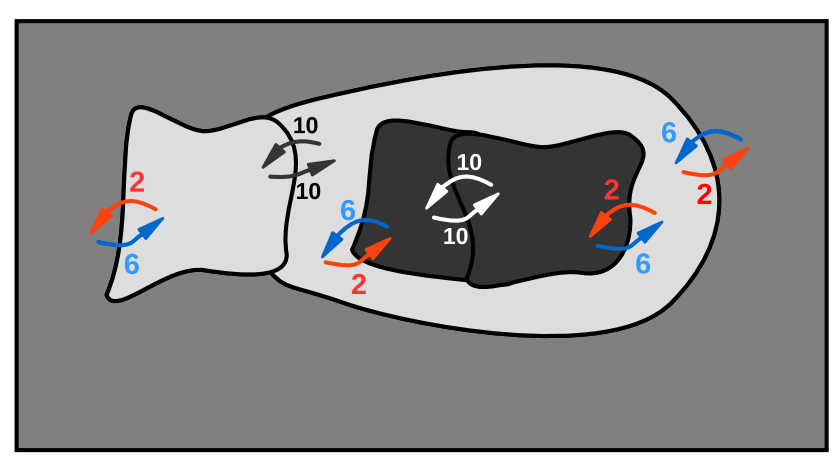

(a) $G=(\mathcal{V}, E, w)$

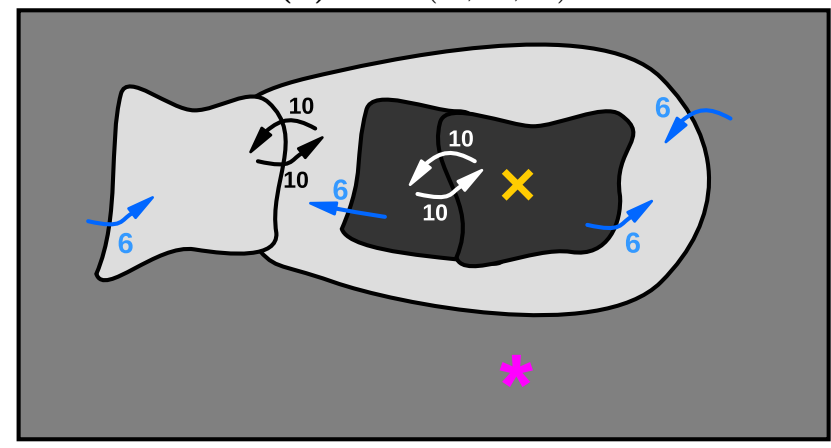

(c) $G_{\leq}$

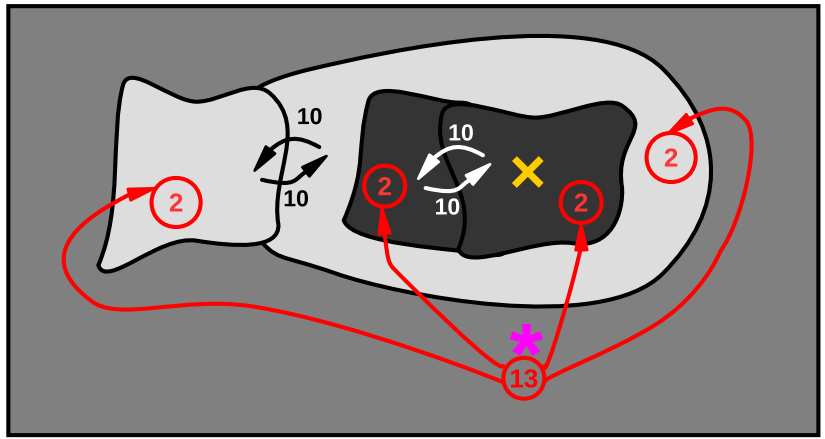

(b) $V_{b}^{K}\left(s_{i}\right)=2$

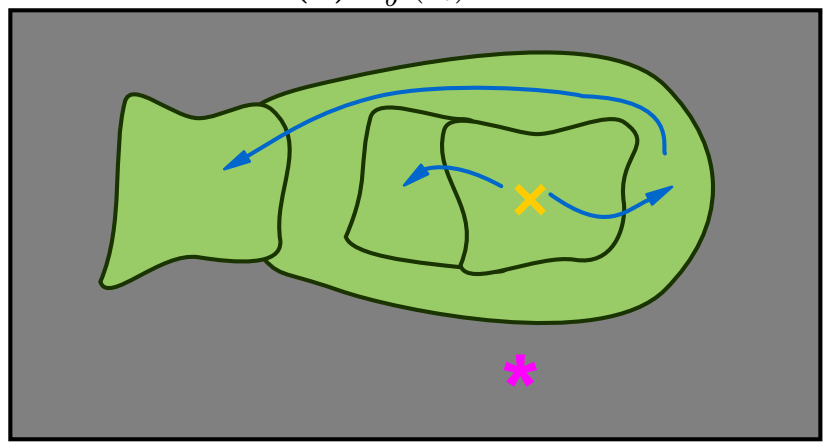

(d) Resultado

Figura 4.6: Algoritmo $A_{O R F C}^{\text {out, }, \times}\left(\left\{s_{i}\right\}, \mathcal{S}_{b}\right)$. Grafo a partir de uma imagem com semente de objeto $\times$ e semente de fundo *, (a) dígrado com fator de orientação $\alpha=0.5$ (b) resutado do cálculo do mapa de conexidade $V_{b}^{W}\left(s_{i}\right)=2$ para uma função de conexidade $f_{\text {min }}^{\nVdash \mathcal{S}_{b}}$, (c) obtenção do grafo residual $G_{\leq},(\mathbf{d})$ o objeto composto pelos pixels que pertencem ao $\mathrm{DCC}\left(\mathrm{s}_{i}\right)$.

Para provar a corretude dos algoritmos acima, precisamos do seguinte lema:

Lema 1. Para um dado dígrafo ponderado $G$, e conjuntos de sementes $\mathcal{S}_{o}$ e $\mathcal{S}_{b}$, tal que $\mathcal{S}_{o}=\left\{s_{i}\right\}$, então $\varepsilon_{\infty \downarrow}^{\text {in }}=V_{b}\left(s_{i}\right)$ e $\varepsilon_{\infty \downarrow}^{\text {out }}=V_{b}^{K}\left(s_{i}\right)$.

Demonstração. Vamos provar o Lema 1 para $\varepsilon_{\infty \downarrow}^{\text {in }}=V_{b}\left(s_{i}\right)$, mas o caso $\varepsilon_{\infty \downarrow}^{\text {out }}=V_{b}^{K}\left(s_{i}\right)$ tem uma prova essencialmente idêntica. A prova é baseada na seguinte afirmação: (1) Para o dado dígrafo fortemente conexo $G$, se removermos todos os $\operatorname{arcos}\langle a, b\rangle$, tal que $w(a, b)<\varepsilon_{\infty \downarrow \downarrow}^{\text {in }}$, nós então obtemos um novo dígrafo $G^{\prime}$ no qual ainda existe um caminho de $\mathcal{S}_{b}$ para $s_{i}$ (isto é, $\exists \pi_{t \rightsquigarrow s_{i}}$ onde $t \in \mathcal{S}_{b}$ ).

Esta afirmação pode ser comprovada por meio de prova por contradição. Seja $T$ o conjunto de pixels alcançáveis a partir de $\mathcal{S}_{b} \mathrm{em} G^{\prime}$ (isto é, $T=\bigcup_{x \in \mathcal{S}_{b}} D C C_{G^{\prime}}(x)$ ). Se não houver caminho de $\mathcal{S}_{b}$ a $s_{i}$ em $G^{\prime}$, então $s_{i} \notin T$. Portanto, temos uma partição dos vértices em dois conjuntos disjuntos $T$ e $\mathcal{V} \backslash T$. Note que os correspondentes arcos de corte $\langle a, b\rangle \in \mathcal{C}_{i n}\left(\chi_{\mathcal{V} / T}\right)$ todos têm $w(a, b)<\varepsilon_{\infty \downarrow}^{i n}$ em $G$. Consequentemente, $\mathcal{C}_{i n}\left(\chi_{\mathcal{V} / T}\right)$ tem um melhor valor de corte do que $\varepsilon_{\infty \downarrow \downarrow}^{i n}$, o que é uma contradição pela Equação 4.10.

Da afirmação (1), podemos concluir que existe um caminho de $\mathcal{S}_{b}$ a $s_{i}$ em $G$, que é composto apenas por $\operatorname{arcos}\langle a, b\rangle: w(a, b) \geq \varepsilon_{\infty \downarrow}^{i n}$. Portanto, o valor de conexidade $V_{b}\left(s_{i}\right)$ de um caminho ótimo de $\mathcal{S}_{b}$ para $s_{i}$ (Eq. 3.2) não pode ser menor do que $\varepsilon_{\infty \downarrow \downarrow}^{i n}$, isto é, $V_{b}\left(s_{i}\right) \geq \varepsilon_{\infty \downarrow}^{i n}(\mathbf{2})$.

Considere o conjunto de arcos de corte $\mathcal{C}_{i n}\left(x_{\text {opt }}\right)$ de uma solução ótima $x_{\text {opt }} \in \mathcal{X}_{\infty}^{\text {in }}\left(\left\{s_{i}\right\}, \mathcal{S}_{b}\right)$. Por definição (Equações 4.8 e 4.10), $w(a, b) \leq \varepsilon_{\infty \downarrow \downarrow}^{i n}$ para todo $\langle a, b\rangle \in \mathcal{C}_{i n}\left(x_{o p t}\right)$. Um caminho ótimo $\pi_{S_{b} \leadsto s_{i}}$, indo de $\mathcal{S}_{b}$ para $s_{i}$, deve, necessariamente, passar por algum arco de $\mathcal{C}_{\text {in }}\left(x_{\text {opt }}\right)$. Logo o seu valor de conexidade $f_{\min }^{\mathcal{S}_{b}}\left(\pi_{S_{b} \rightsquigarrow s_{i}}\right)=V_{b}\left(s_{i}\right)$ não pode ser maior do que $\varepsilon_{\infty \downarrow}^{\text {in }}$, isto é, $V_{b}\left(s_{i}\right) \leq \varepsilon_{\infty \downarrow}^{\text {in }}(\mathbf{3})$.

A partir das condições anteriores (2) e (3), podemos concluir que a única configuração válida é $V_{b}\left(s_{i}\right)=\varepsilon_{\infty \downarrow}^{i n}$.

Por uma questão de simplicidade, só vamos discutir aqui a prova de corretude do algoritmo 
$A_{O R F C}^{i n, \text {, }}\left(\left\{s_{i}\right\}, S_{b}\right)$, em termos da Equação 4.15, onde $s_{1}=s_{i}$ e $\varepsilon_{\infty \downarrow}^{i n}=V_{b}\left(s_{i}\right)$ (Lema 1). O algoritmo para $A_{O R F C}^{\text {out,ox }}\left(\left\{s_{i}\right\}, S_{b}\right)$ tem uma prova essencialmente idêntica.

Primeiro, precisamos provar que a função característica $\chi_{O}$ de $O=D C C_{G_{\leq}^{T}}\left(s_{i}\right)$ é uma solução ótima em $\mathcal{X}_{\infty}^{\text {in }}\left(\left\{s_{i}\right\}, \mathcal{S}_{b}\right)$. Note que, no dígrafo $G_{\leq}^{T}$, não existem arcos apontando de pixels em $D C C_{G_{<}^{T}}\left(s_{i}\right)$ para pixels em $\mathcal{V} \backslash D C C_{G_{<}^{T}}\left(s_{i}\right)$, caso contrário, a lista de sucessores de $s_{i}$ em $G_{\leq}^{T}$, dada por $\bar{D} C C_{G_{\leq}^{T}}\left(s_{i}\right)$, não estaria completa. Estes arcos foram removidos no Passo 2 do algoritmo $A_{O R F C}^{i n, \propto<}\left(\left\{s_{i}\right\}, S_{b}\right)$, e portanto não têm valores maiores do que $\varepsilon_{\infty \downarrow}^{i n}$, de modo que a função característica de $D C C_{G_{\leq}^{T}}\left(s_{i}\right)$ deve ser uma solução ótima em $\mathcal{X}_{\infty}^{\text {in }}\left(\left\{s_{i}\right\}, \mathcal{S}_{b}\right)$.

As outras condições da Equação 4.15 forçam $A_{O R F C}^{i n, \propto<}\left(\left\{s_{i}\right\}, S_{b}\right)$ a constituir o menor objeto em $\mathcal{X}_{\infty}^{\text {in }}\left(\left\{s_{i}\right\}, \mathcal{S}_{b}\right)$. Note que qualquer objeto composto por um conjunto de pixels $T$, de tal modo que existem arcos de pixels em $T$ para pixels em $\mathcal{V} \backslash T$ no dígrafo $G_{\leq}^{T}$, não pode ser uma solução ótima em $\mathcal{X}_{\infty}^{\text {in }}\left(\left\{s_{i}\right\}, \mathcal{S}_{b}\right)$, porque estes arcos têm arcos anti-paralelos correspondentes em $G_{\leq}$, que apontam para os pixels do objeto, com valores superiores a $\varepsilon_{\infty \downarrow}^{i n}$, levando a um corte interno pior. Uma vez que todos os subconjuntos próprios de $D C C_{G_{\leq}^{T}}\left(s_{i}\right)$ ainda possuem alguns arcos de saída no dígrafo $G_{\leq}^{T}$, e, consequentemente, arcos de entrada em $G_{\leq}$, então eles não são ótimos. Portanto, $D C C_{G_{\leq}^{T}}\left(s_{i}\right)$ é a menor solução ótima.

Para resolver o caso do $A_{O R F C}^{\text {in, }<<}\left(\mathcal{S}_{o}, \mathcal{S}_{b}\right)$ com múltiplas sementes internas, de acordo com a Equação 4.16, precisamos repetir a execução do Algoritmo 3 para cada semente interna. No entanto, a seguinte proposição se aplica no caso do $A_{O R F C}^{i n, \propto<}\left(\mathcal{S}_{o}, \mathcal{S}_{b}\right)$ :

Proposição 1. Para um dado dígrafo $G=(\mathcal{V}, E, w)$ e semente $s_{i} \in \mathcal{S}_{o}$, considere o dígrafo residual $G_{s_{i}}=G_{\leq}=\left(\mathcal{V},\left\{\langle a, b\rangle \in E: w(a, b)>V_{b}\left(s_{i}\right)\right\}, w\right)$ (Etapa 2 do Algoritmo 3). Para quaisquer sementes arbitrárias $s_{1} \in \mathcal{S}_{o}$ e $s_{2} \in \mathcal{S}_{o}$, se $V_{b}\left(s_{1}\right) \leq V_{b}\left(s_{2}\right)$ e $s_{2} \in D C C_{G_{s_{1}}^{T}}\left(s_{1}\right)$, então $D C C_{G_{s_{2}}^{T}}\left(s_{2}\right) \subset D C C_{G_{s_{1}}^{T}}\left(s_{1}\right)$.

Se ordenamos as sementes $s_{i}$ em $\mathcal{S}_{o}$ de acordo com seus valores $V_{b}\left(s_{i}\right)$, então nós podemos processar as sementes em ordem crescente de valores, o que nos permite evitar o reprocessamento de pixels. Durante o processamento das sementes, podemos pular as sementes correspondentes a pixels que já foram processados e atribuídos ao objeto, melhorando consideravelmente o tempo de execução e tornando a complexidade do algoritmo independente do número de sementes usadas.

Um ponto importante a ser observado é o fato que $O R F C$ engloba $R F C$ como um caso particular sempre que o parâmetro $\alpha$ é definido como zero.

\subsection{Análise dos métodos ORFC e OIFT}

Nesta seção vamos analisar as regiões de robustez das sementes internas, comparando os métodos ORFC e OIFT. Para isso precisamos das seguintes definições:

Definição 2 (Núcleo de uma semente interna $s_{i}$ ). A região de robustez de uma semente interna $s_{i}$, também conhecida como núcleo, é a região onde podemos mover a semente $s_{i}$ sem alterar sua segmentação correspondente.

Definição 3 (Sementes equivalentes). Duas sementes internas $s_{1}$ e $s_{2}$ são ditas equivalentes se elas separadamente produzem o mesmo resultado de segmentação. Isto é, para um dado algoritmo $A\left(\mathcal{S}_{o}, \mathcal{S}_{b}\right)$ e conjunto de sementes externas $\mathcal{S}_{b}$, o resultado $A\left(\left\{s_{1}\right\}, \mathcal{S}_{b}\right)=A\left(\left\{s_{2}\right\}, \mathcal{S}_{b}\right)$.

A noção de equivalência de sementes introduzida na Definição 3 é uma relação binária $\equiv$ no conjunto de pixels do objeto $O$, tal que $A\left(\left\{s_{1}\right\}, \mathcal{S}_{b}\right)=\chi_{O}$. Isto é, $s_{1} \equiv s_{2}$ se e somente se $s_{1}$ e $s_{2}$ são equivalentes. Essa relação é reflexiva, simétrica e transitiva, portanto ela é de fato uma relação de equivalência conforme definido na matemática. Portanto o núcleo de uma semente $s_{1} \mathrm{em} \equiv$, 
denotado por $\left[s_{1}\right]$, é definido como $\left[s_{1}\right]=\left\{t \in O: s_{1} \equiv t\right\}$.

Usaremos a notação $\mathcal{N}_{A}\left(\left\{s_{1}\right\}, \mathcal{S}_{b}\right)=\chi_{T}: T=\left[s_{1}\right]$ para indicar o núcleo de $s_{1}$ pelo algorimo $A$. No caso do método RFC, $\mathcal{N}_{R F C}\left(\left\{s_{1}\right\}, \mathcal{S}_{b}\right)=A_{R F C}\left(\left\{s_{1}\right\}, \mathcal{S}_{b}\right)$ [Saha e Udupa (2001)].

Definição 4 (Operação de união e inclusão de subconjuntos fuzzy). Para duas funções características fornecidas $\chi_{O_{1}}$ e $\chi_{O_{2}}$, vamos usar a notação $\chi_{O_{1}} \leq \chi_{O_{2}}$ para indicar que $O_{1} \subseteq O_{2}$ (isto é, $\chi_{O_{1}}(a) \leq \chi_{O_{2}}(a)$ para todo $\left.a \in \mathcal{V}\right)$.

Vamos usar também $\chi_{O_{1} \cup O_{2}}=\max \left\{\chi_{O_{1}}, \chi_{O_{2}}\right\}$ (isto é, $\chi_{O_{1} \cup O_{2}}(a)=\max \left\{\chi_{O_{1}}(a), \chi_{O_{2}}(a)\right\}$ para todo $a \in \mathcal{V})$ [Kaufmann (1975)].

Proposição 2. Sejam $\chi_{O_{O R F C}}=A_{O R F C}^{\text {out,ox }}\left(\mathcal{S}_{o}, \mathcal{S}_{b}\right)$ e $\chi_{O_{O I F T}}=A_{O I F T}^{\text {out }}\left(\mathcal{S}_{o}, \mathcal{S}_{b}\right)$. Para quaisquer conjuntos de sementes $\mathcal{S}_{o}$ e $\mathcal{S}_{b}, O_{O R F C} \subseteq O_{O I F T}\left(\right.$ isto é $\left.A_{O R F C}^{\text {out,ox }}\left(\mathcal{S}_{o}, \mathcal{S}_{b}\right) \leq A_{O I F T}^{\text {out }}\left(\mathcal{S}_{o}, \mathcal{S}_{b}\right)\right)$.

Demonstração. O caso com única semente interna pode ser provado facilmente com base em suas definições. Pela Equação 4.13, sabemos que $A_{O R F C}^{\text {out,se }}\left(\left\{s_{1}\right\}, \mathcal{S}_{b}\right) \in \mathcal{X}_{\infty}^{\text {out }}\left(\left\{s_{1}\right\}, \mathcal{S}_{b}\right)$ e baseado nas referências [Mansilla e Miranda (2013a); Miranda e Mansilla (2014)] $A_{O I F T}^{\text {out }}\left(\left\{s_{1}\right\}, \mathcal{S}_{b}\right) \in \mathcal{X}_{\infty}^{\text {out }}\left(\left\{s_{1}\right\}, \mathcal{S}_{b}\right)$. Como as duas segmentações estão contidas no mesmo conjunto $\mathcal{X}_{\infty}^{\text {out }}\left(\left\{s_{1}\right\}, \mathcal{S}_{b}\right)$ e dado que $A_{O R F C}^{\text {out,ox }}\left(\left\{s_{1}\right\}, \mathcal{S}_{b}\right)$ é o menor representante da família $\mathcal{X}_{\infty}^{\text {out }}\left(\left\{s_{1}\right\}, \mathcal{S}_{b}\right)$ (Equação 4.13), então $A_{O R F C}^{\text {out,ox }}\left(\left\{s_{1}\right\}, \mathcal{S}_{b}\right) \leq A_{O I F T}^{\text {out }}\left(\left\{s_{1}\right\}, \mathcal{S}_{b}\right)$.

No caso de múltiplas sementes internas, de acordo com a Equação 4.14:

$$
A_{O R F C}^{\text {out, },<}\left(\mathcal{S}_{o}, \mathcal{S}_{b}\right)=\max _{s_{i} \in \mathcal{S}_{o}}\left\{A_{O R F C}^{\text {out, ,e }}\left(\left\{s_{i}\right\}, \mathcal{S}_{b}\right)\right\}
$$

Utilizando o fato que $A_{O R F C}^{\text {out, se }}\left(\left\{s_{i}\right\}, \mathcal{S}_{b}\right) \leq A_{O I F T}^{\text {out }}\left(\left\{s_{i}\right\}, \mathcal{S}_{b}\right)$, podemos concluir que:

$$
\max _{s_{i} \in \mathcal{S}_{o}}\left\{A_{O R F C}^{\text {out, }, \propto<}\left(\left\{s_{i}\right\}, \mathcal{S}_{b}\right)\right\} \leq \max _{s_{i} \in \mathcal{S}_{o}}\left\{A_{O I F T}^{\text {out }}\left(\left\{s_{i}\right\}, \mathcal{S}_{b}\right)\right\}
$$

As Figuras 4.7 e 4.8 mostram que a união dos objetos via OIFT obtidos usando cada semente interna separadamente pode ser menor que o resultado combinado da OIFT usando todas as sementes de uma só vez, isto é:

$$
\max _{s_{i} \in \mathcal{S}_{o}}\left\{A_{O I F T}^{\text {out }}\left(\left\{s_{i}\right\}, \mathcal{S}_{b}\right)\right\} \leq A_{O I F T}^{\text {out }}\left(\mathcal{S}_{o}, \mathcal{S}_{b}\right)
$$

Note que os melhores valores de caminho $f_{O I F T}^{\mathcal{S}_{b}, \mathcal{S}_{o}}$ oferecidos a partir das sementes internas podem piorar no caso de removermos sementes de $\mathcal{S}_{o}$. Portanto uma segmentação $A_{O I F T}^{\text {out }}\left(\mathcal{S}_{o}^{\prime}, \mathcal{S}_{b}\right)$, usando menos sementes (isto é, $\mathcal{S}_{o}^{\prime} \subset \mathcal{S}_{o}$ ), pode apenas encolher, isto é $A_{O I F T}^{\text {out }}\left(\mathcal{S}_{o}^{\prime}, \mathcal{S}_{b}\right) \leq A_{O I F T}^{\text {out }}\left(\mathcal{S}_{o}, \mathcal{S}_{b}\right)$.

Então a Equação 4.19 é verdadeira, pois se $A_{O I F T}^{\text {out }}\left(\left\{s_{i}\right\}, \mathcal{S}_{b}\right) \leq A_{O I F T}^{\text {out }}\left(\mathcal{S}_{o}, \mathcal{S}_{b}\right)$ para todo $s_{i} \in \mathcal{S}_{o}$, então a união dos resultados das sementes internas individuas só pode estar contida em $A_{O I F T}^{\text {out }}\left(\mathcal{S}_{o}, \mathcal{S}_{b}\right)$. Portanto, juntando as Equações 4.17, 4.18 e 4.19:

$$
A_{O R F C}^{\text {out, se }}\left(\mathcal{S}_{o}, \mathcal{S}_{b}\right) \leq A_{O I F T}^{\text {out }}\left(\mathcal{S}_{o}, \mathcal{S}_{b}\right)
$$




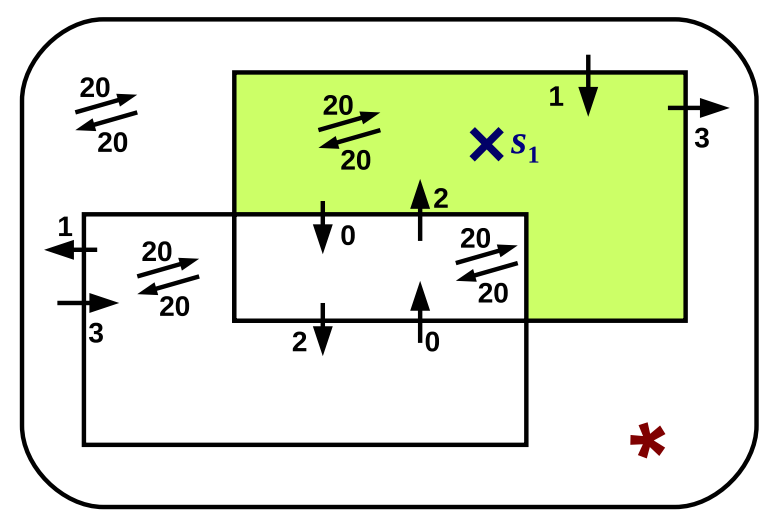

(a) $A_{O R F C}^{\text {out,ose }}\left(\left\{s_{1}\right\}, \mathcal{S}_{b}\right)$

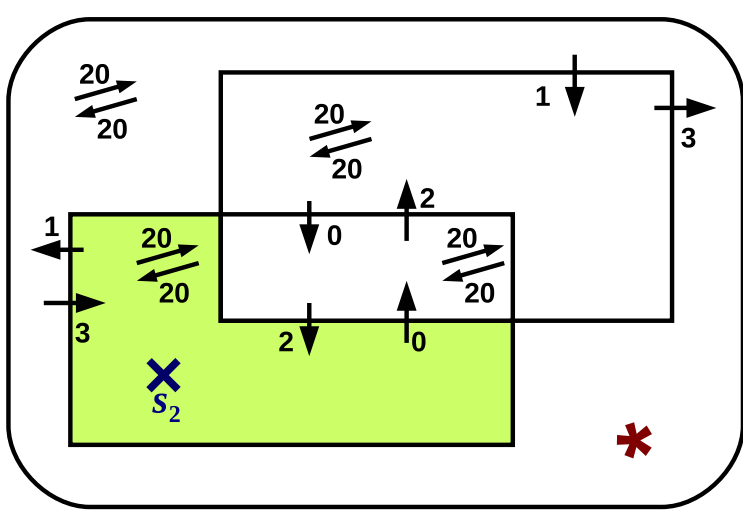

(b) $A_{O R F C}^{\text {out,oce }}\left(\left\{s_{2}\right\}, \mathcal{S}_{b}\right)$

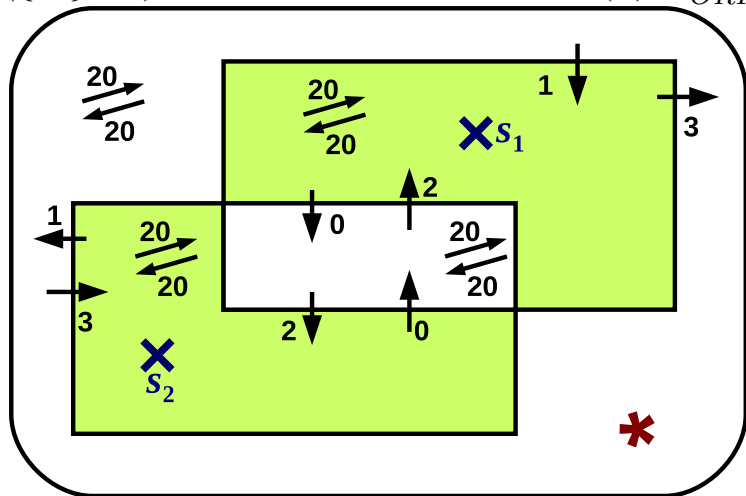

(c) $A_{O R F C}^{\text {out, se }}\left(\left\{s_{1}, s_{2}\right\}, \mathcal{S}_{b}\right)$

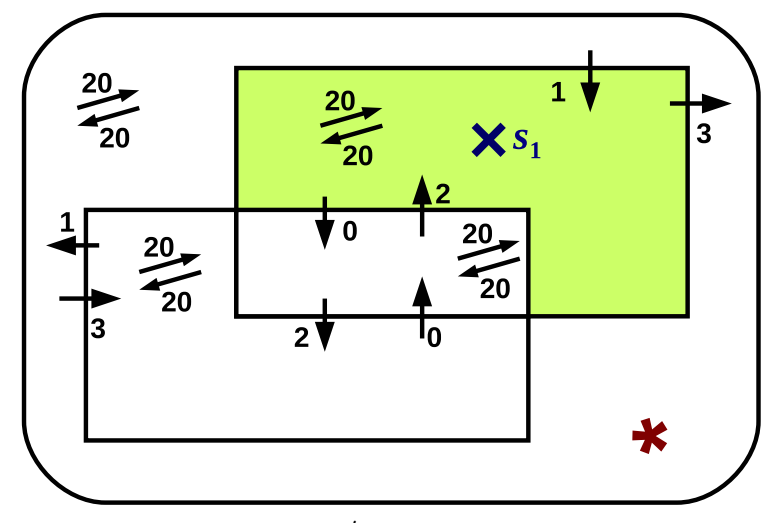

(d) $A_{O I F T}^{\text {out }}\left(\left\{s_{1}\right\}, \mathcal{S}_{b}\right)$

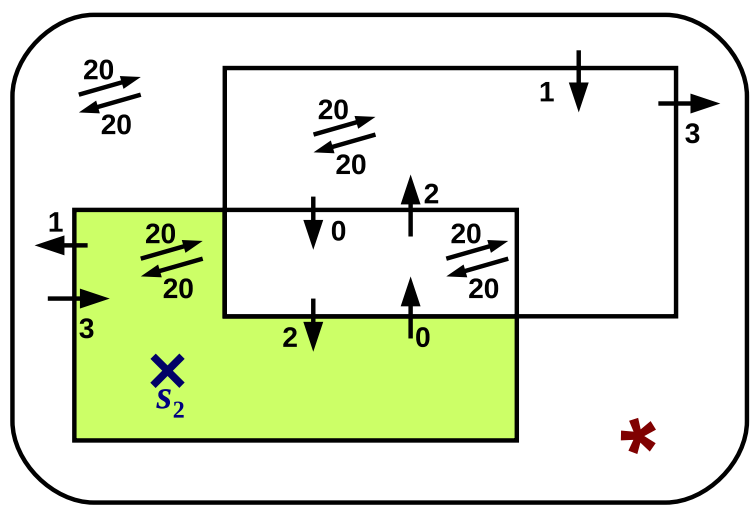

(e) $A_{O I F T}^{\text {out }}\left(\left\{s_{2}\right\}, \mathcal{S}_{b}\right)$

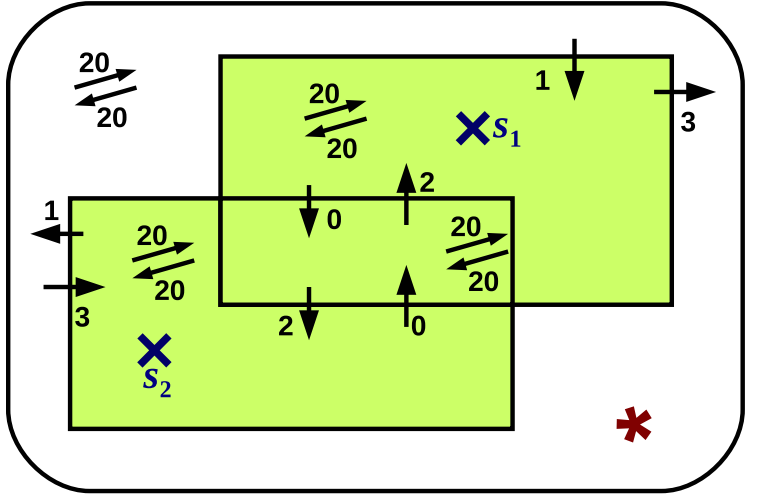

(f) $A_{O I F T}^{\text {out }}\left(\left\{s_{1}, s_{2}\right\}, \mathcal{S}_{b}\right)$

Figura 4.7: Exemplo de um grafo direcionado com semente do objeto em diferentes posições, onde $\times$ representa a semente de objeto $e \star$ a semente de fundo. (a) O resultado da segmentação usando o algoritmo $A_{O R F C}^{\text {out,ec }}\left(\left\{s_{1}\right\}, \mathcal{S}_{b}\right)$, (b) segmentação usando o algoritmo $A_{O R F C}^{\text {out, }<}\left(\left\{s_{2}\right\}, \mathcal{S}_{b}\right)$, (c) segmentação usando o algoritmo $A_{O R F C}^{\text {out,se }}\left(\left\{s_{1}, s_{2}\right\}, \mathcal{S}_{b}\right)$ com duas sementes no objeto. (d) Resultado de segmentação usando $A_{O I F T}^{\text {out }}\left(\left\{s_{1}\right\}, \mathcal{S}_{b}\right)$, (e) segmentação usando $A_{O I F T}^{\text {out }}\left(\left\{s_{2}\right\}, \mathcal{S}_{b}\right)$, (f) resultado de segmentação usando $A_{O I F T}^{\text {out }}\left(\left\{s_{1}, s_{2}\right\}, \mathcal{S}_{b}\right)$ com duas sementes no objeto. 


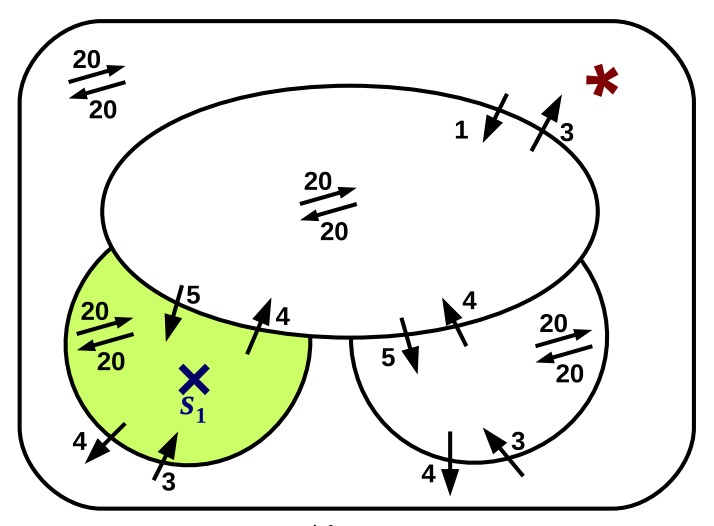

(a) $A_{O R F C}^{\text {out,ox }}\left(\left\{s_{1}\right\}, \mathcal{S}_{b}\right)$

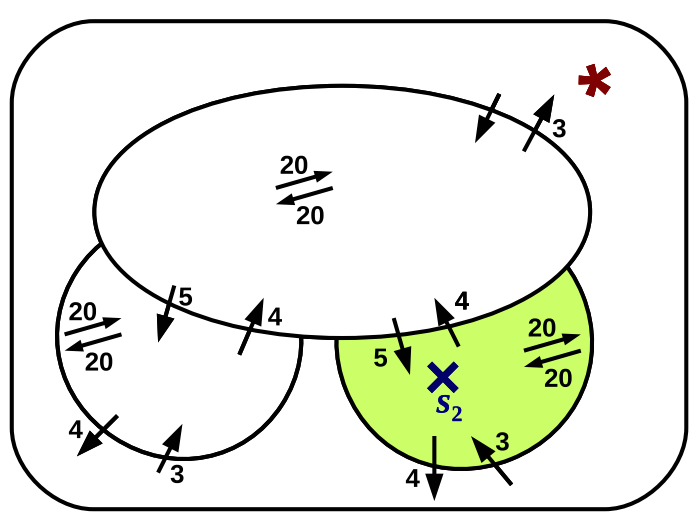

(b) $A_{O R F C}^{\text {out,oce }}\left(\left\{s_{2}\right\}, \mathcal{S}_{b}\right)$



(c) $A_{O R F C}^{\text {out,oc }}\left(\left\{s_{1}, s_{2}\right\}, \mathcal{S}_{b}\right)$

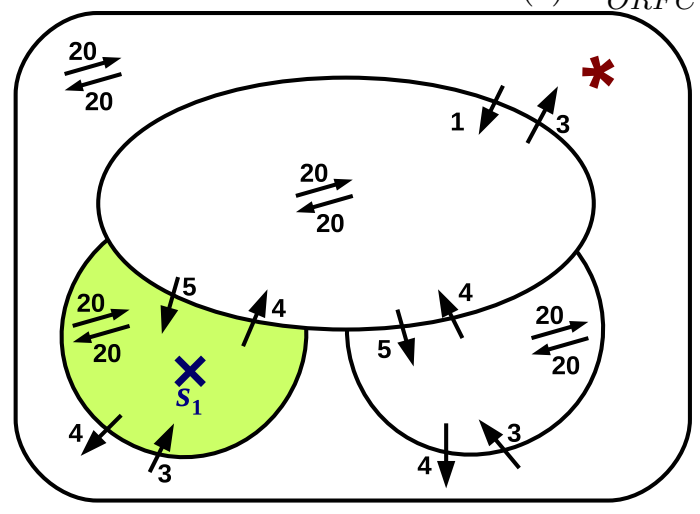

(d) $A_{O I F T}^{\text {out }}\left(\left\{s_{1}\right\}, \mathcal{S}_{b}\right)$

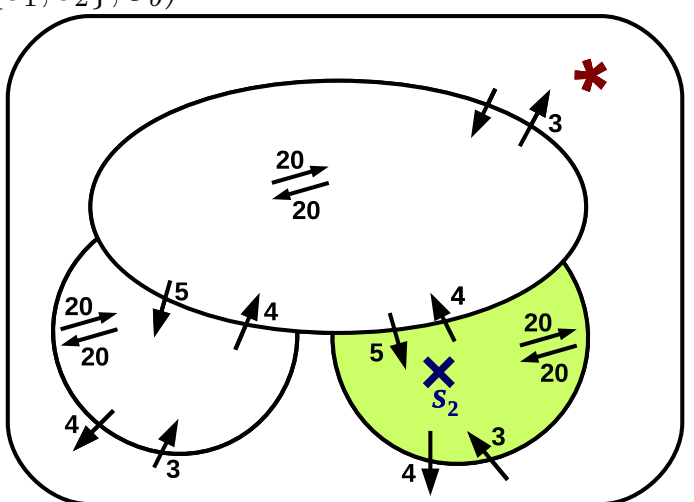

(e) $A_{O I F T}^{\text {out }}\left(\left\{s_{2}\right\}, \mathcal{S}_{b}\right)$

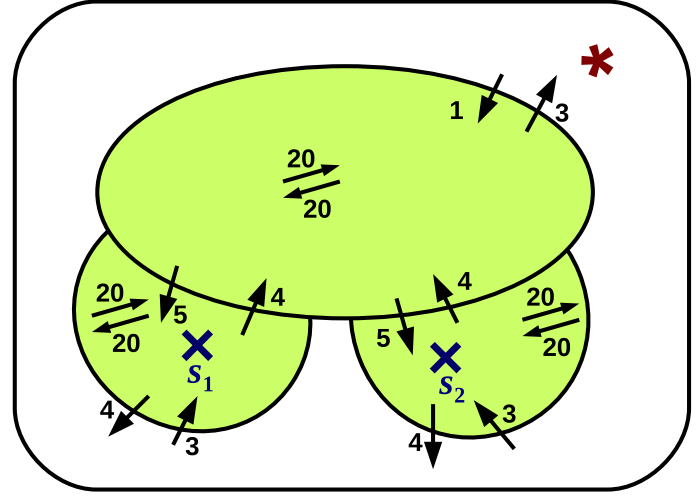

(f) $A_{O I F T}^{\text {out }}\left(\left\{s_{1}, s_{2}\right\}, \mathcal{S}_{b}\right)$

Figura 4.8: Exemplo de um grafo direcionado com semente do objeto em diferentes posições, onde $\times$ representa a semente de objeto $e \star$ a semente de fundo. (a) $O$ resultado da segmentação usando o algoritmo $A_{O R F C}^{\text {out, se }}\left(\left\{s_{1}\right\}, \mathcal{S}_{b}\right)$, (b) segmentação usando o algoritmo $A_{O R F C}^{\text {out, s× }}\left(\left\{s_{2}\right\}, \mathcal{S}_{b}\right)$, (c) segmentação usando o algoritmo $A_{O R F C}^{\text {out, s< }}\left(\left\{s_{1}, s_{2}\right\}, \mathcal{S}_{b}\right)$ com duas sementes no objeto. (d) Resultado de segmentação usando $A_{O I F T}^{\text {out }}\left(\left\{s_{1}\right\}, \mathcal{S}_{b}\right),(\mathbf{e})$ segmentação usando $A_{O I F T}^{\text {out }}\left(\left\{s_{2}\right\}, \mathcal{S}_{b}\right)$, (f) resultado de segmentação usando $A_{O I F T}^{\text {out }}\left(\left\{s_{1}, s_{2}\right\}, \mathcal{S}_{b}\right)$ com duas sementes no objeto. 
A seguir é apresentada uma análise dos núcleos dos métodos. Dado que o objeto delineado pelo método RFC corresponde ao núcleo da IFT-Watershed [Audigier e Lotufo (2007); Miranda et al. (2010a)], uma primeira pergunta a ser respondida consiste em saber se, de modo análogo, o resultado do ORFC é o núcleo da OIFT. Na Figura 4.9, é apresentado um contraexemplo, portanto ORFC não é o núcleo da OIFT.

Outra pergunta pertinente consiste em saber se os algoritmos ORFC e OIFT possuem o mesmo núcleo. A Figura 4.10 apresenta um contraexemplo, portanto o núcleo da ORFC é diferente do núcleo da OIFT.

Outra questão interessante consiste em saber se o núcleo da ORFC-CoH (ORFC seguido de um pós-processamento por fechamento de buracos - Close of Holes) corresponde ao núcleo da OIFT, mas novamente temos um contraexemplo, conforme apresentado na Figura 4.11.

Os resultados das Figuras 4.9, 4.10 e 4.11 nos sugerem a seguinte proposição:

Proposição 3. Para qualquer semente interna $s_{1}$ e conjunto de sementes externas $\mathcal{S}_{b}$ :

$$
\mathcal{N}_{\text {ORFC }}^{\text {out, },<}\left(\left\{s_{1}\right\}, \mathcal{S}_{b}\right) \leq \mathcal{N}_{\mathrm{CoH}(\mathrm{ORFC})}^{\text {out,o< }}\left(\left\{s_{1}\right\}, \mathcal{S}_{b}\right) \leq \mathcal{N}_{O I F T}^{\text {out }}\left(\left\{s_{1}\right\}, \mathcal{S}_{b}\right)
$$

Demonstração. A primeira parte da Equação 4.20, $\mathcal{N}_{O R F C}^{\text {out,ox }}\left(\left\{s_{1}\right\}, \mathcal{S}_{b}\right) \leq \mathcal{N}_{\operatorname{CoH}(\text { ORFC })}^{\text {out,ox }}\left(\left\{s_{1}\right\}, \mathcal{S}_{b}\right)$, possui prova trivial, uma vez que qualquer posição de uma semente que resulte no mesmo objeto de saída via ORFC, também produzirá necessariamente a mesma saída com fechamento de buracos por definição.

$\mathcal{N}_{O R F C}^{\text {out,oce }}\left(\left\{s_{1}\right\}, \mathcal{S}_{b}\right) \leq \mathcal{N}_{\text {OIFT }}^{\text {out }}\left(\left\{s_{1}\right\}, \mathcal{S}_{b}\right)$ pode ser provado da seguinte forma:

$$
C_{\text {ORFC }}=\mathcal{C}_{\text {out }}\left(A_{O R F C}^{\text {out, }, \times<}\left(\left\{s_{1}\right\}, \mathcal{S}_{b}\right)\right)
$$

$\mathrm{e}$

$$
C_{O I F T}=\mathcal{C}_{\text {out }}\left(A_{O I F T}^{\text {out }}\left(\left\{s_{1}\right\}, \mathcal{S}_{b}\right)\right)
$$

sabemos que $w(a, b) \leq \varepsilon_{\infty \downarrow}^{\text {out }}$ para qualquer arco em $C_{O R F C} \cup C_{O I F T}$ (Equações 4.7 e 4.9).

Uma vez que o resultado de $A_{O R F C}^{\text {out,se }}\left(\left\{s_{1}\right\}, \mathcal{S}_{b}\right)$ corresponde ao menor elemento da família $\mathcal{X}_{\infty}^{\text {out }}\left(\left\{s_{1}\right\}, \mathcal{S}_{b}\right)$, então $\forall c \in O: \chi_{O}=A_{O R F C}^{\text {out,o< }}\left(\left\{s_{1}\right\}, \mathcal{S}_{b}\right)$ existe um caminho $\pi_{s_{1} \rightsquigarrow c}$ que passa exclusivamente por arcos com pesos maiores que $\varepsilon_{\infty \downarrow}^{\text {out }}$ (isto é, $\pi_{s_{1} \rightsquigarrow c}=\left\langle t_{1}=s_{1}, t_{2}, \ldots, t_{n}=c\right\rangle$ e $w\left(t_{i}, t_{i+1}\right)>\varepsilon_{\infty \downarrow}^{\text {out }}$ para $\left.i=1, \ldots, n-1\right)$, caso contrário $c$ não faria parte do $D C C_{G \leq}\left(s_{1}\right)$ (Algoritmo 4).

Logo, para qualquer arco $\langle a, b\rangle \in C_{O R F C}$ :

$$
f_{O I F T}^{\mathcal{S}_{b},\left\{s_{1}\right\}}\left(\pi_{s_{1} \rightsquigarrow a}^{P}\right)>2 \times \varepsilon_{\infty \downarrow}^{\text {out }}+1
$$

$\mathrm{e}$

$$
f_{O I F T}^{\mathcal{S}_{b},\left\{s_{1}\right\}}\left(\pi_{s_{1} \rightsquigarrow a}^{P} \cdot\langle a, b\rangle\right)=2 \times w(a, b)+1 \leq 2 \times \varepsilon_{\infty \downarrow}^{o u t}+1
$$

onde $\pi_{s_{1} \rightsquigarrow a}^{P}$ corresponde ao caminho calculado pela IFT com função $f_{O I F T}^{\mathcal{S}_{b},\left\{s_{1}\right\}}$ na floresta $P$.

Portanto, os valores de $f_{O I F T}^{\mathcal{S}_{b},\left\{s_{1}\right\}}$ oferecidos para pixels externos ao corte $C_{O R F C}$ são definidos estritamente pelos valores dos arcos em $C_{O R F C}$. Logo podemos concluir que o resultado do $A_{O I F T}^{\text {out }}\left(\left\{s_{1}\right\}, \mathcal{S}_{b}\right)$ não é afetado por mudanças na posição da semente $s_{1}$ dentro do núcleo do ORFC, pois as propriedades anteriores continuarão válidas dado que teremos o mesmo corte $C_{O R F C}$. 
$\mathcal{N}_{\text {CoH }(\text { ORFC })}^{\text {out }}\left(\left\{s_{1}\right\}, \mathcal{S}_{b}\right) \leq \mathcal{N}_{O I F T}^{\text {out }}\left(\left\{s_{1}\right\}, \mathcal{S}_{b}\right)$ pode ser provado de maneira análoga usando essencialmente os mesmos argumentos.

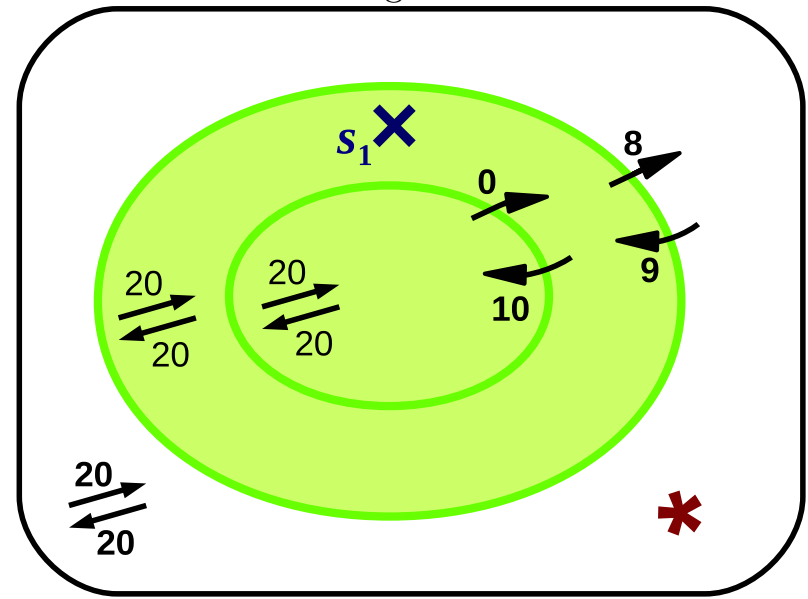

(a) $A_{O R F C}^{\text {out,ose }}\left(\left\{s_{1}\right\}, \mathcal{S}_{b}\right)$

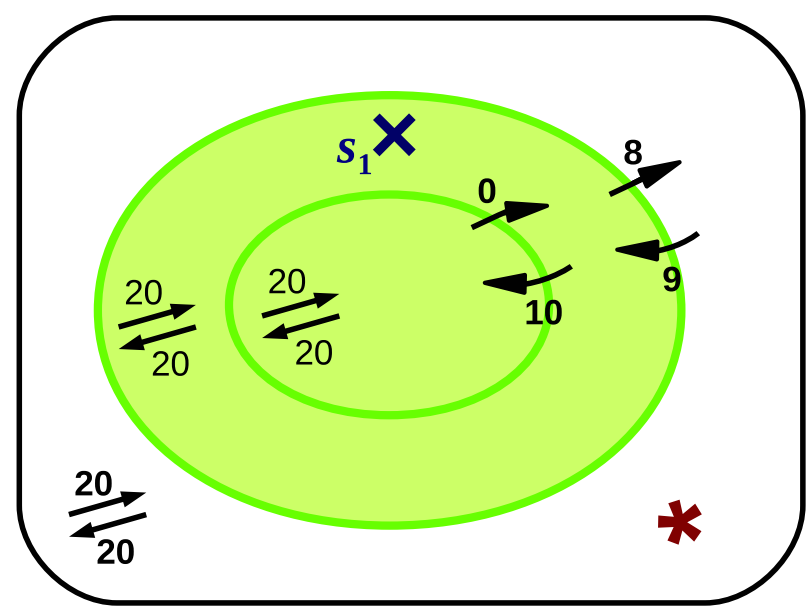

(c) $A_{O I F T}^{\text {out }}\left(\left\{s_{1}\right\}, \mathcal{S}_{b}\right)$



(e) $\mathcal{N}_{O R F C}\left(\left\{s_{1}\right\}, \mathcal{S}_{b}\right)$

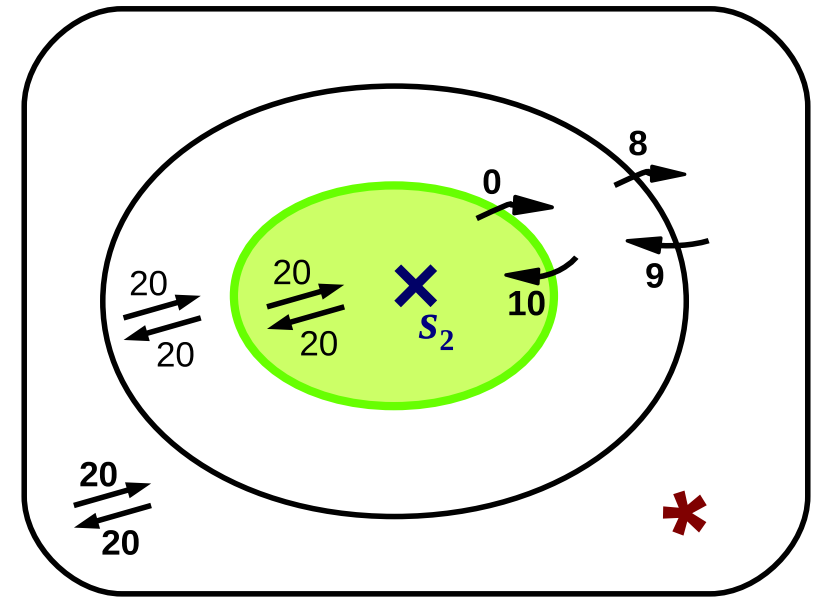

(b) $A_{O R F C}^{\text {out, se }}\left(\left\{s_{2}\right\}, \mathcal{S}_{b}\right)$

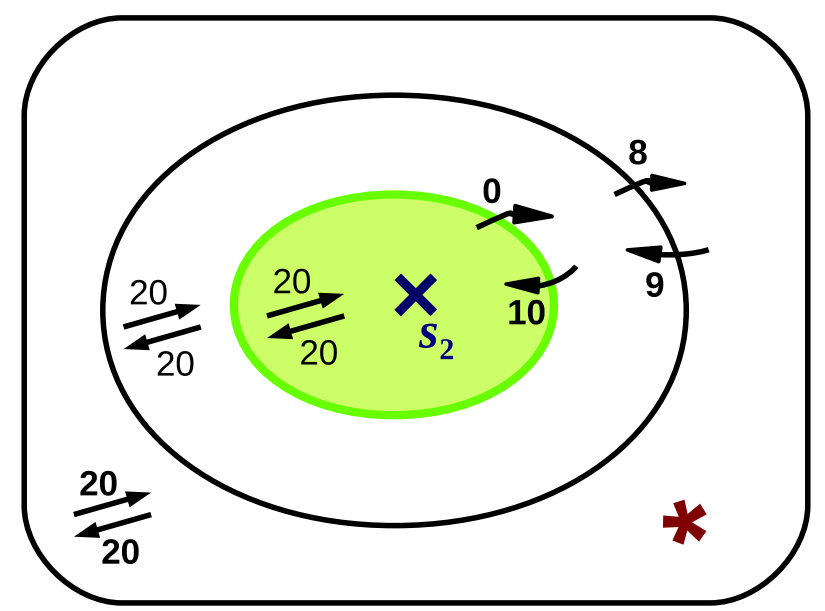

(d) $A_{O I F T}^{\text {out }}\left(\left\{s_{2}\right\}, \mathcal{S}_{b}\right)$

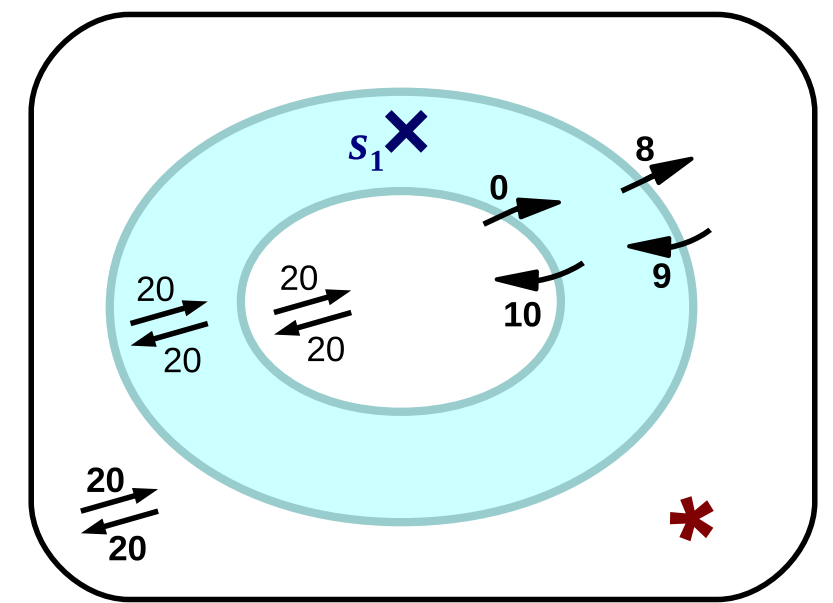

(f) $\mathcal{N}_{\text {OIFT }}\left(\left\{s_{1}\right\}, \mathcal{S}_{b}\right)$

Figura 4.9: Exemplo de um grafo direcionado com semente do objeto em diferentes posições, onde $\times$ representa a semente de objeto $e \star$ a semente de fundo. (a) $O$ resultado da segmentação com o algoritmo $A_{O R F C}^{\text {out, se }}\left(\left\{s_{1}\right\}, \mathcal{S}_{b}\right)$, onde $V_{b}^{W}\left(s_{1}\right)=8,(\mathbf{b})$ o algoritmo $A_{O R F C}^{\text {out, }, \text { a }}\left(\left\{s_{2}\right\}, \mathcal{S}_{b}\right)$ onde $V_{b}^{\mathbb{W}}\left(s_{2}\right)=0$ no item (b). (c-d) Os resultados de segmentação com o algoritmo $A_{O I F T}^{\text {out }}\left(\left\{s_{i}\right\}, \mathcal{S}_{b}\right)$. Note que os algoritmos ORFC e OIFT apresentam o mesmo resultado de segmentação. (e) Região de robustez de $s_{1}$ para ORFC, (f) região de robustez de $s_{1}$ para OIFT. 


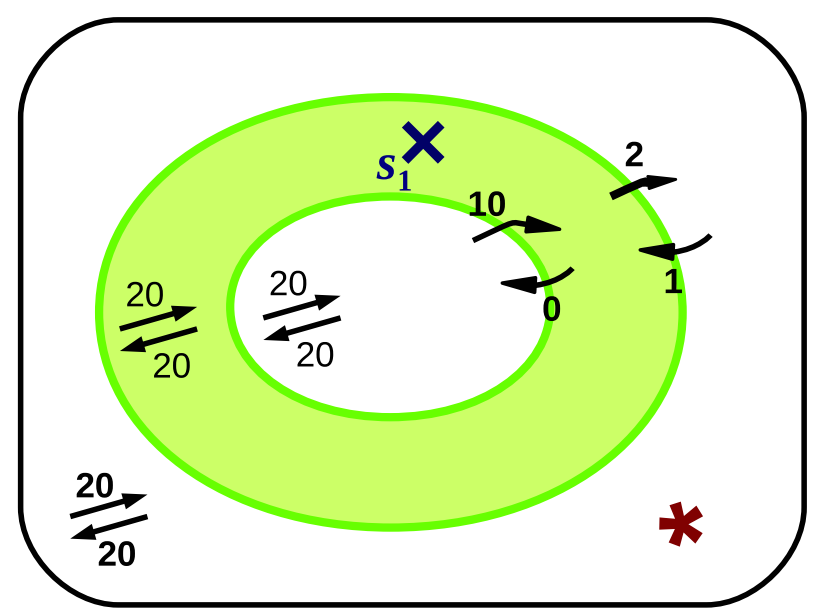

(a) $A_{O R F C}^{\text {out, se }}\left(\left\{s_{1}\right\}, \mathcal{S}_{b}\right)$

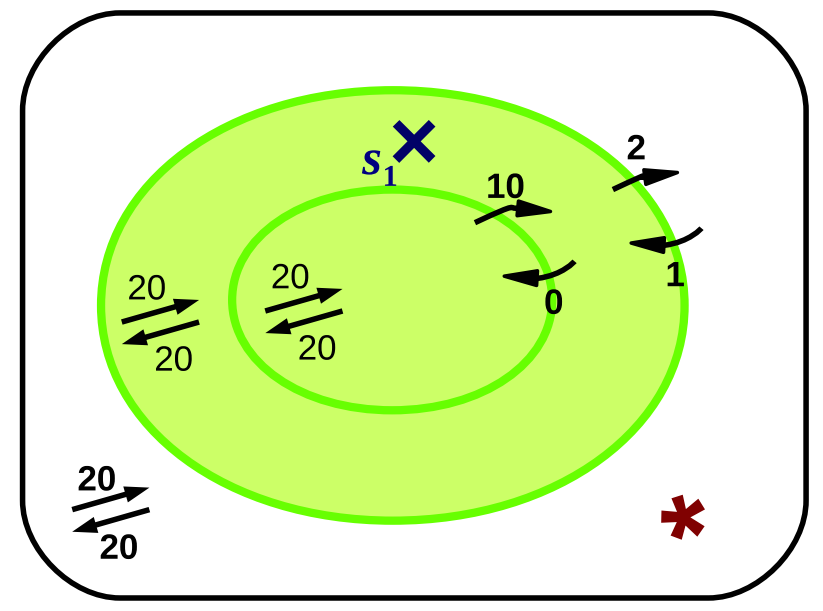

(c) $A_{O I F T}^{\text {out }}\left(\left\{s_{1}\right\}, \mathcal{S}_{b}\right)$



(c) $\mathcal{N}_{O R F C}^{\text {out, }, \propto<}\left(\left\{s_{1}\right\}, \mathcal{S}_{b}\right)$

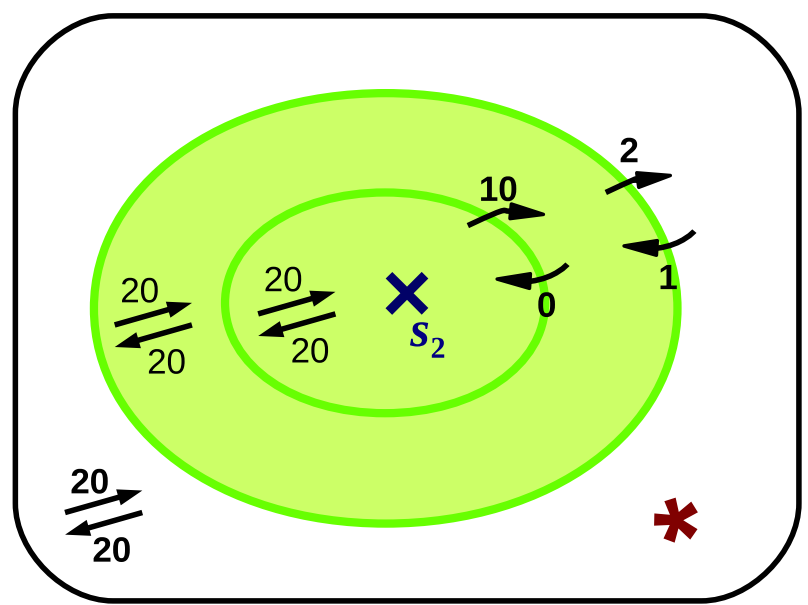

(b) $A_{O R F C}^{\text {out, ox }}\left(\left\{s_{2}\right\}, \mathcal{S}_{b}\right)$

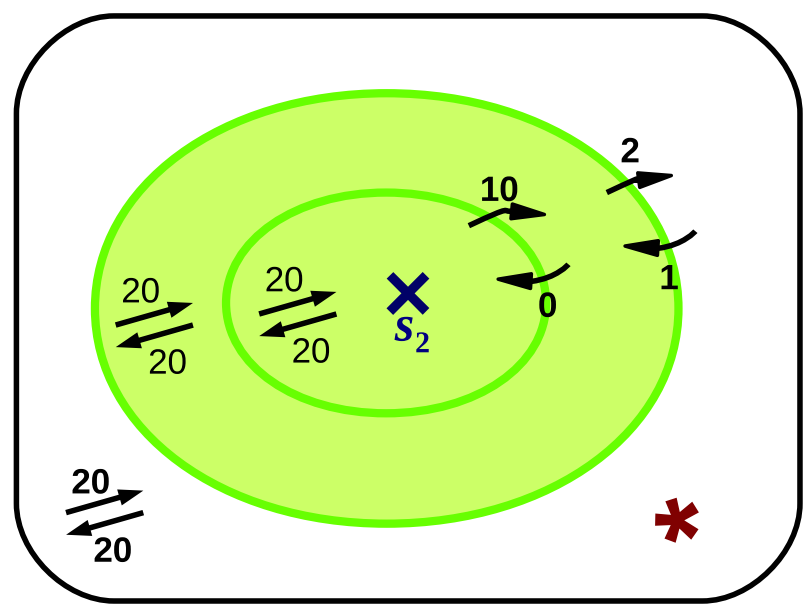

(d) $A_{O I F T}^{\text {out }}\left(\left\{s_{2}\right\}, \mathcal{S}_{b}\right)$

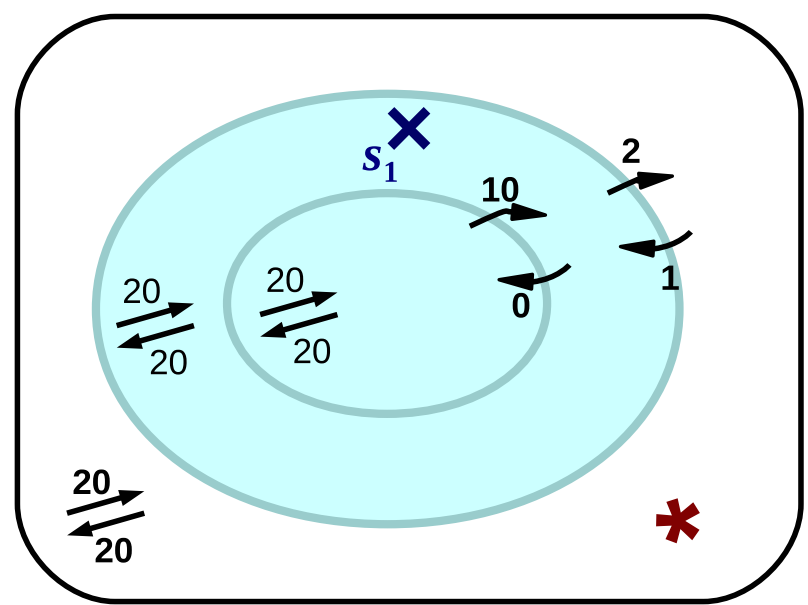

(d) $\mathcal{N}_{O I F T}^{\text {out }}\left(\left\{s_{1}\right\}, \mathcal{S}_{b}\right)$

Figura 4.10: Exemplo de um grafo direcionado com semente do objeto em diferentes posições, onde $\times$ representa a semente de objeto $e \star$ a semente de fundo. (a-b) O resultado da segmentação com o algoritmo $A_{O R F C}^{\text {out, },<}\left(\left\{s_{1}\right\}, \mathcal{S}_{b}\right)$, onde $V_{b}^{W}\left(s_{1}\right)=2$ no item $(\mathbf{a})$, e o algoritmo $A_{O R F C}^{\text {out, , }}\left(\left\{s_{2}\right\}, \mathcal{S}_{b}\right)$ onde $V_{b}^{W}\left(s_{2}\right)=2$ no item (b). (c-d) Os resultados de segmentação com o algoritmo $A_{O I F T}^{\text {out }}\left(\left\{s_{i}\right\}, \mathcal{S}_{b}\right)$. 


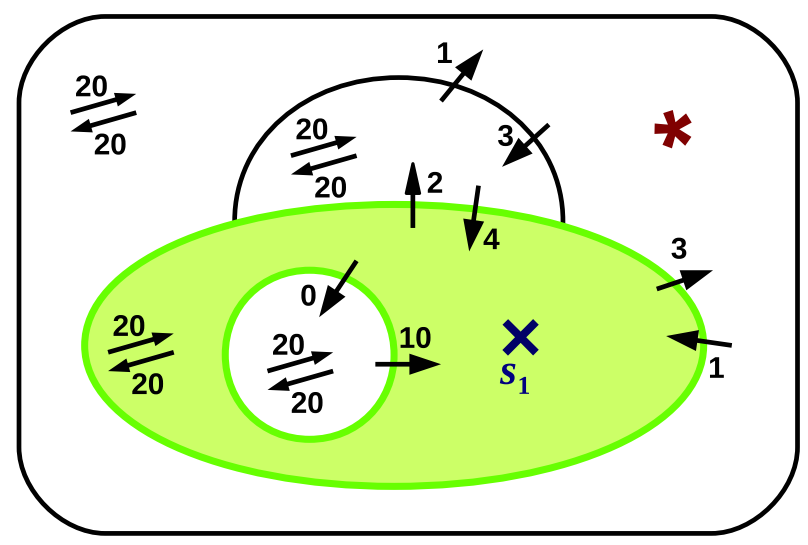

(a) $A_{O R F C}^{\text {out, se }}\left(\left\{s_{1}\right\}, \mathcal{S}_{b}\right)$

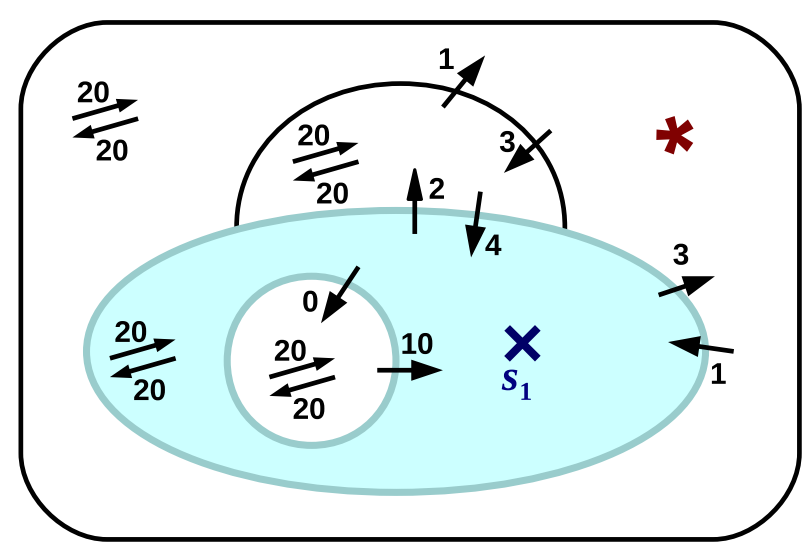

(b) $\mathcal{N}_{O R F C}^{\text {out, },<}\left(\left\{s_{1}\right\}, \mathcal{S}_{b}\right)$

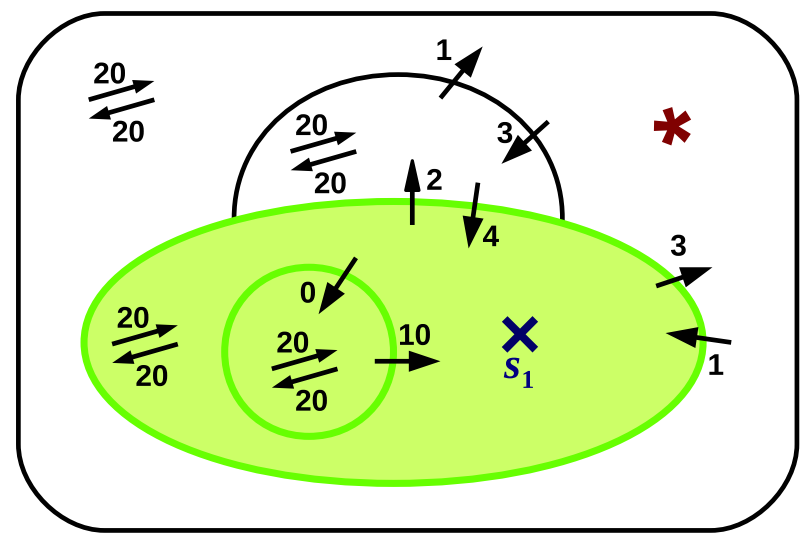

(c) $\operatorname{CoH}\left(A_{O R F C}^{\text {out, ,e }}\left(\left\{s_{1}\right\}, \mathcal{S}_{b}\right)\right)$

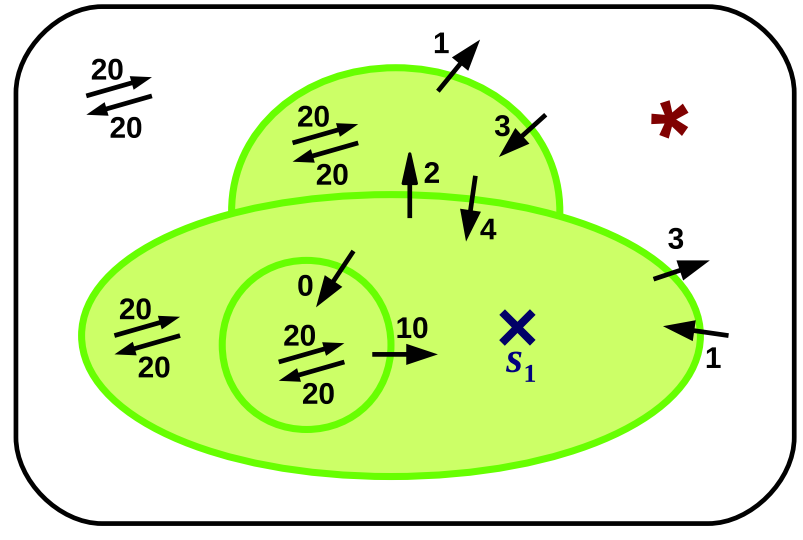

(d) $A_{O I F T}^{\text {out }}\left(\left\{s_{1}\right\}, \mathcal{S}_{b}\right)$

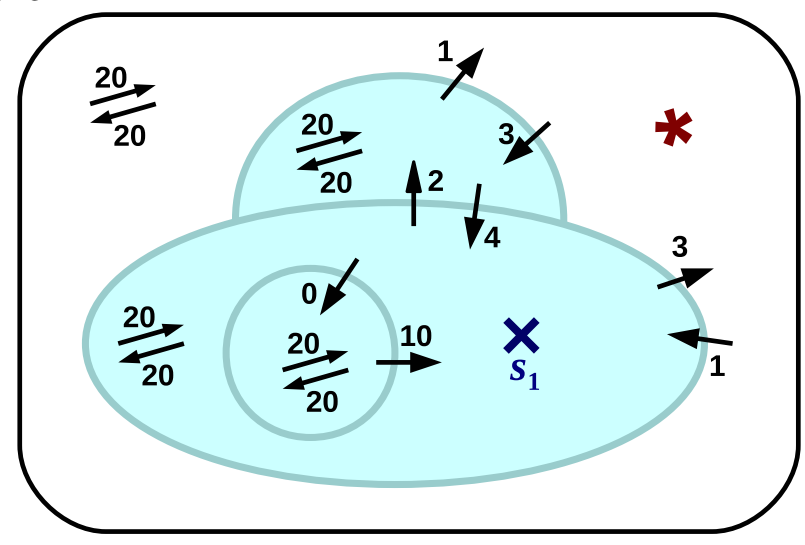

(e) $\mathcal{N}_{O I F T}^{\text {out }}\left(\left\{s_{1}\right\}, \mathcal{S}_{b}\right)$

Figura 4.11: Exemplo de um grafo direcionado, onde $\times$ representa a semente de objeto $e \star$ a semente de fundo. (a) O resultado da segmentação usando o algoritmo $A_{O R F C}^{\text {out, }, \times<}\left(\left\{s_{1}\right\}, \mathcal{S}_{b}\right)$, (b) o núcleo do ORFC $\mathcal{N}_{O R F C}^{\text {out, }, \propto}\left(\left\{s_{1}\right\}, \mathcal{S}_{b}\right),(\mathbf{c})$ resultado de segmentação usando $A_{O R F C}^{\text {out,æe }}\left(\left\{s_{1}\right\}, \mathcal{S}_{b}\right)$ com fechamento de buraco $($ CoH $)$, (d) resultado de segmentação usando $A_{O I F T}^{\text {out }}\left(\left\{s_{1}\right\}, \mathcal{S}_{b}\right)$, (e) núcleo do OIFT $\mathcal{N}_{O I F T}^{\text {out }}\left(\left\{s_{1}\right\}, \mathcal{S}_{b}\right)$.

\subsection{Método Híbrido ORFC+GC}

Nesta seção, nós seguimos a mesma ideia apresentada em Ciesielski et al. (2013) e propomos uma nova abordagem híbrida que combina os pontos fortes dos algoritmos conexidade fuzzy relativa com orientação - ORFC (Capítulo 4) e o método de corte em grafo - GC (Seção 3.4) em dígrafos. 


\subsubsection{Características dos métodos ORFC \& GC}

Os algoritmos RFC e GC têm seus pontos fortes e fracos complementares. As características mais importantes entre os algoritmos correspondem a sensibilidade em sua resposta em relação a escolha de sementes e a suavidade da borda do objeto segmentado/delineado [Ciesielski et al. (2013)]

- Velocidade: O algoritmo RFC/ORFC executa mais rápida do que o GC. A estimativa teórica do pior caso em tempo de execução do RFC/ORFC, é $O(n)$ ou $O(n \log (n))$ dependendo da implementação da fila de prioridade utilizada [Ciesielski et al. (2012a)]. Com respeito ao tamanho da imagem ( $n=N \times M$ sendo $N$ o número de linhas e $M$ o número de colunas), enquanto que o tempo de execução do GC é de ordem $O\left(n^{3}\right)$ ou $O\left(n^{2.5}\right)$ para a implementação mais rápida [Boykov e Kolmogorov (2004)].

- Problema de viés de encolhimento: O algoritmo GC tem a tendência de selecionar os objetos com tamanho muito pequeno de borda, até mesmo quando os pesos das arestas da borda são altos. RFC/ORFC não apresenta problema de encolhimento.

- Robustez: O resultado do algoritmo RFC não é afetado por alterações da posição das sementes, pois a região de robustez do RFC corresponde a sua região delineada. Já o resultado do GC pode tornar-se sensível até mesmo para pequenas alterações das sementes. No caso do ORFC, temos em geral que o seu núcleo é menor do que a sua região delineada (Figura 4.9a e Figura 4.9e), no entanto a sua região de robustez é mais estável que a do GC, devido a ausência do problema de encolhimento no ORFC.

O método de corte em grafo (GC) suporta nativamente a restrição de polaridade de borda, quando é executado em dígrafos, sendo denotado como corte em grafo com orientação (OGC - Oriented Graph Cut) no caso de dígrafos. Ele $\left(A_{O G C}^{\text {out }}\left(S_{o}, S_{b}\right)\right)$ resolve o problema da $\varepsilon_{1}$-minimização considerando os arcos que limitam o fluxo a partir da fonte para o destino, e, consequentemente, minimiza a soma dos pesos dos arcos apontando do objeto para os pixels do fundo (isto é, o corte externo) [Boykov e Funka-Lea (2006)].

A minimização da soma dos arcos do corte interno $\left(A_{O G C}^{i n}\left(S_{o}, S_{b}\right)\right)$ pode ser obtida invertendo os nós de origem e de destino, ou invertendo todos os arcos, calculando GC sobre a transposta do grafo $G^{T}$.

Basicamente, considerando o esquema proposto em Ciesielski et al. (2013), porém usando um grafo ponderado dirigido, com ORFC no lugar do RFC, nós temos a abordagem híbrida ORFC+GC (Figura 4.12) conforme os seguintes algoritmos:

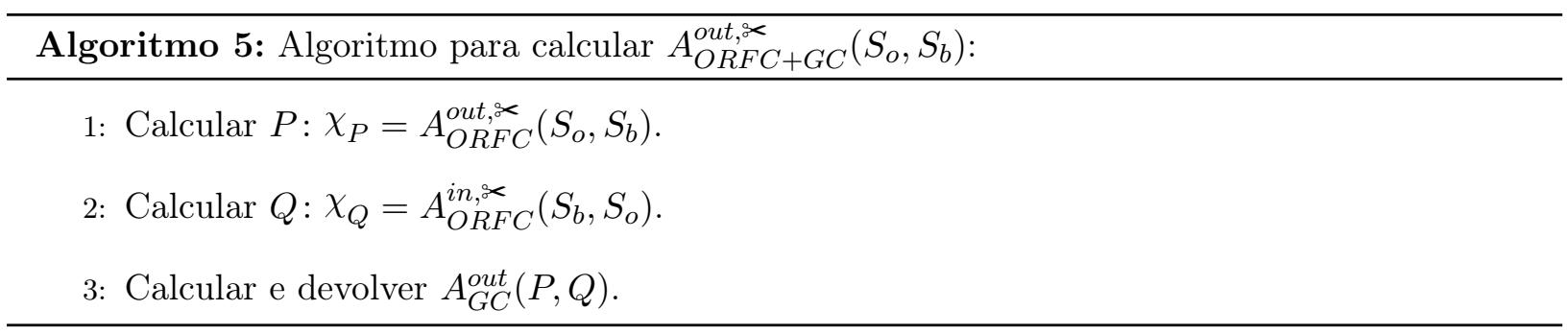


Figura 4.12 ilustra as etapas do Algoritmo 5.
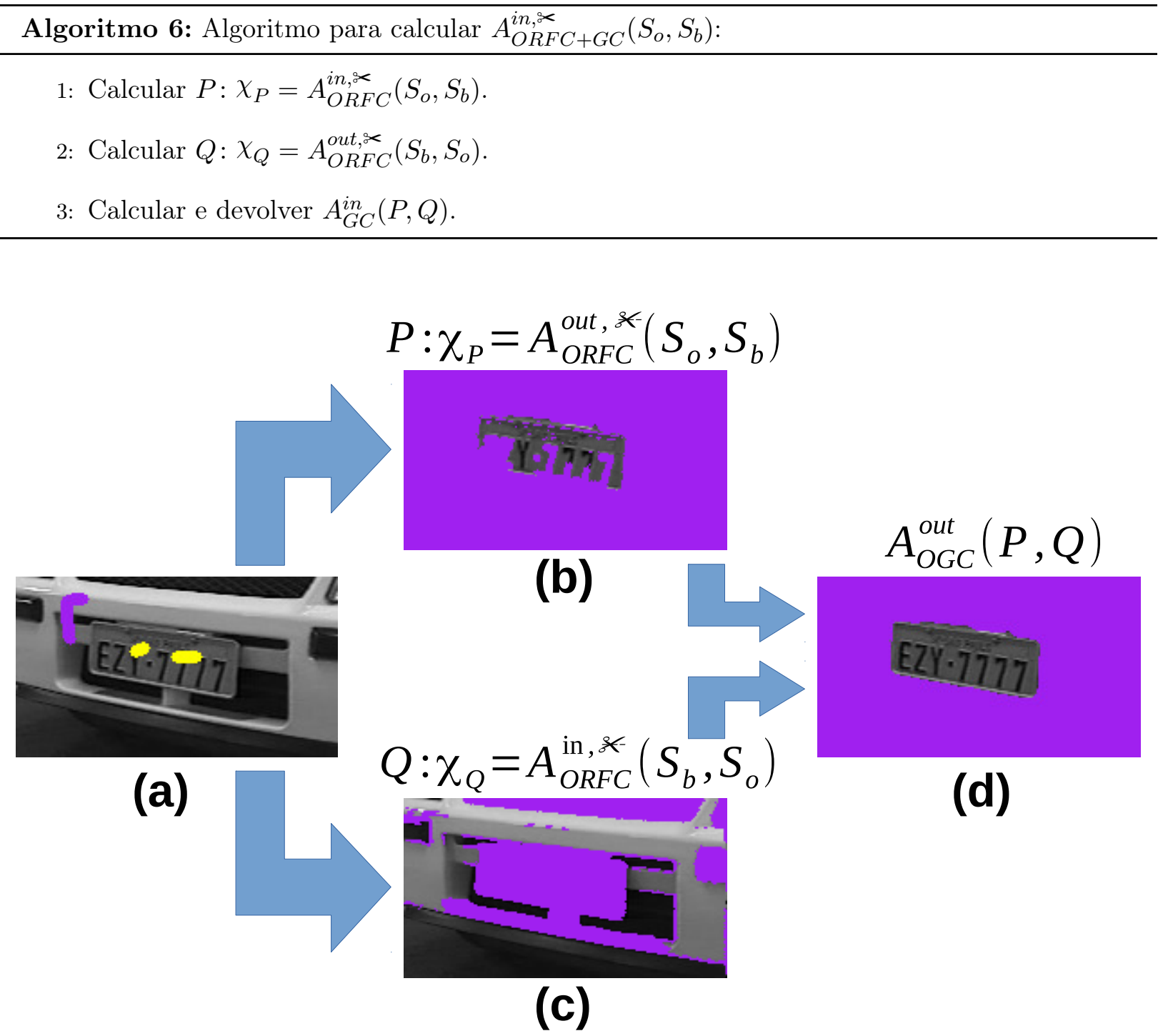

Figura 4.12: Algoritmo $A_{O R F C+G C}^{\text {out, }}\left(\mathcal{S}_{o}, \mathcal{S}_{b}\right)$. (a) Imagem de entrada com sementes $\mathcal{S}_{o}$ e $\mathcal{S}_{b}$. (b) $P: \chi_{P}=A_{O R F C}^{o u t, \text {, }}\left(\mathcal{S}_{o}, \mathcal{S}_{b}\right)$. (c) $Q: \chi_{Q}=A_{O R F C}^{\text {in, }, \times}\left(\mathcal{S}_{b}, \mathcal{S}_{o}\right)$. (d) $A_{G C}^{\text {out }}(P, Q)$.

A ideia é explorar o fato de que o resultado do ORFC apresenta uma baixa taxa de falsos positivos (conforme será mostrado nos experimentos apresentados no próximo capítulo) e a sua maior robustez em relação a posição das sementes. Portanto, usamos o resultado do ORFC de modo a obter um conjunto ampliado de sementes para uma posterior execução do OGC, reduzindo os problemas de viés de encolhimento e reduzindo o tempo de execução, dado que o OGC é executado em uma região menor da imagem, composta apenas pelos pixels que não foram rotulados pelo ORFC. 


\section{Capítulo 5}

\section{Resultados experimentais}

Nesta seção é apresentada uma análise qualitativa e uma análise quantitativa das abordagens propostas e dos métodos de segmentação relacionados realizada em imagens médicas 2-D e 3D [Ciesielski et al. (2013)]. Os experimentos qualitativos utilizam a atribuição de peso baseada na imagem conforme proposto em Miranda et al. (2010b), conforme disponível no nosso software interativo CAOS (Computer-Aided Object Segmentation).

\subsection{Análise qualitativa}

Nos exemplos a seguir apresentamos a eficácia da segmentação da abordagem proposta. Na Figura 5.1 são apresentados exemplos de segmentação, sendo o objeto de interesse o osso rádio em uma imagem por ressonância magnética do punho. Observe que os melhores resultados são obtidos via ORFC+GC (ORFC combinado com o método de Corte em Grafo).

Na Figura 5.2 são apresentados exemplos de segmentação, sendo o objeto de interesse o osso tálus em uma fatia de uma imagem de ressonância magnética de um pé. Observe que o ORFC apresenta uma taxa de erros mais baixa de falsos positivos.

Na Figura 5.3 são apresentados resultados de segmentação do fígado em uma imagem de tomografia computadorizada. Observe que o ORFC produziu resultados superiores que o OIFT.

Para a avaliação em imagens $3 \mathrm{D}^{1}$ foi considerado a tarefa de segmentação do cérebro (Skull Stripping), isto é, eliminar fundo, ossos, olhos, pele e vasos sanguíneos, conforme mostrado na Figura 5.4. Os volumes foram obtidos com um scanner $2 T$ Elscint e com um tamanho de voxel $0.98 \times 0.98 \times 1.00 \mathrm{~mm}^{3}$. O cerebelo é ligado ao resto do cérebro através do tronco cerebral e através do seu topo em função do volume parcial. A ausência de uma fronteira clara entre estas estruturas é um verdadeiro desafio para a segmentação. Observe que o ORFC+GC produziu o resultado mais similar ao do gabarito.

\footnotetext{
${ }^{1}$ imagens do cérebro 3D: imagens da Facultade de Ciências Médicas da UNICAMP, agradecemos ao Prof. Fernando Cendes
} 


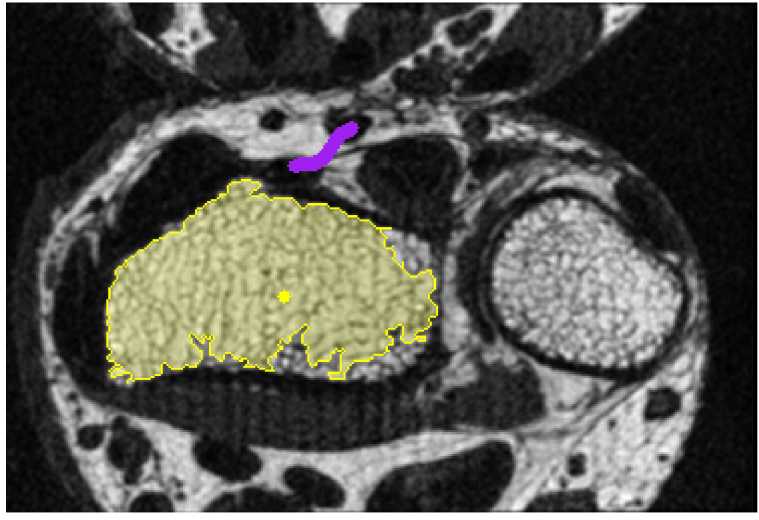

(a)

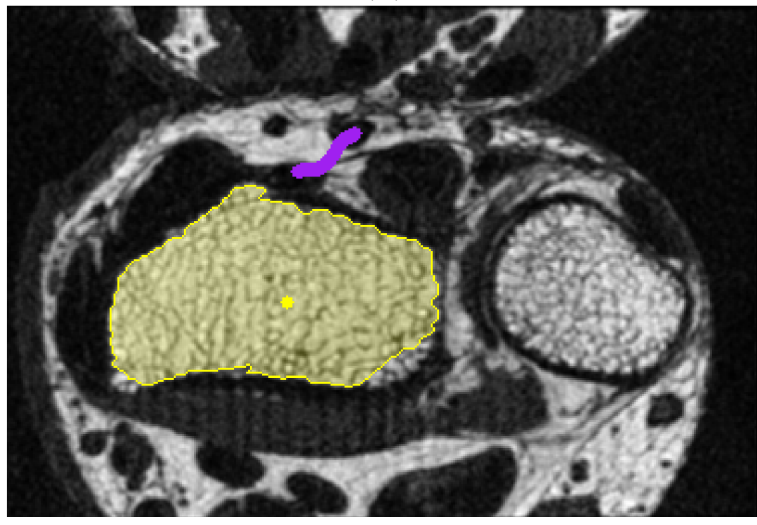

(c)

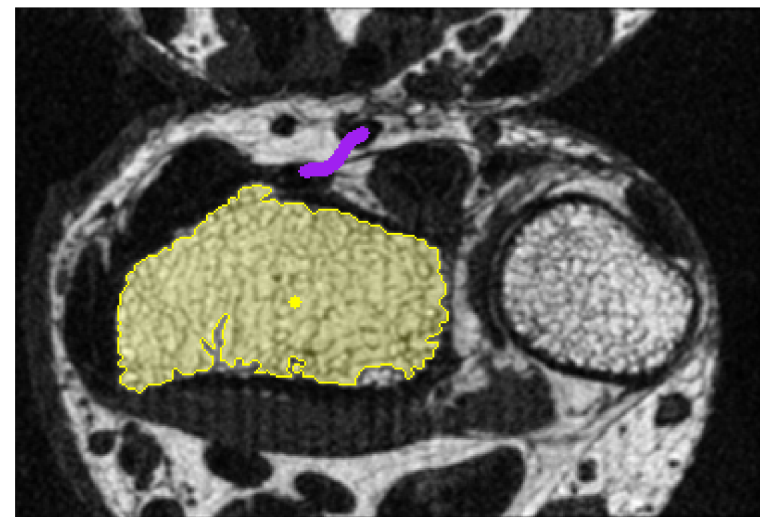

(b)

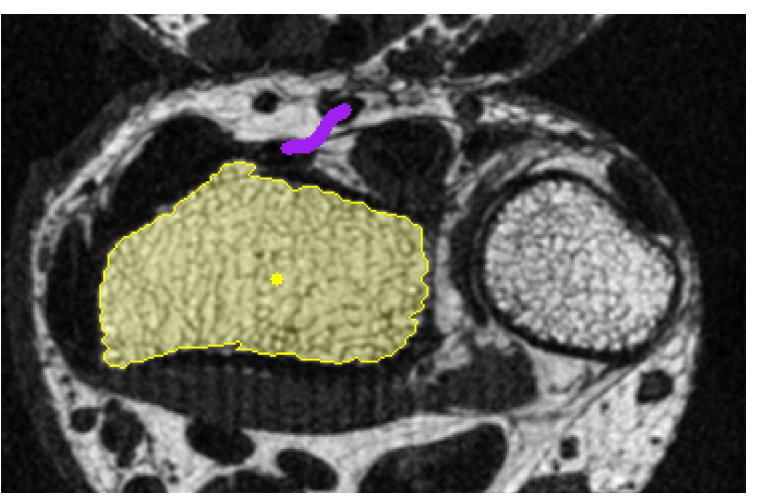

(d)

Figura 5.1: Cortes axiais de ressonância magnética do punho. A segmentação de osso rádio por: (a) IRFC [Ciesielski et al. (2007)], (b) OIFT [Miranda e Mansilla (2014)], (c) RFC+GC [Ciesielski et al. (2013)] e (d) $O R F C+G C$. 


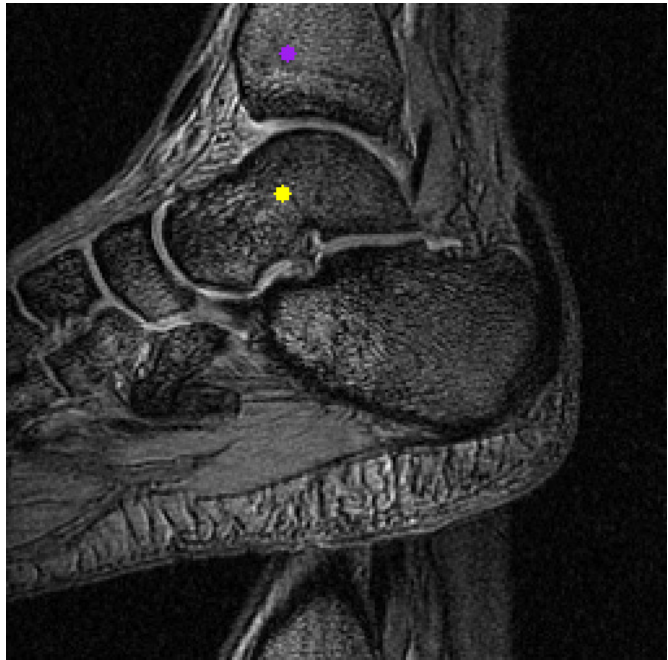

(a)

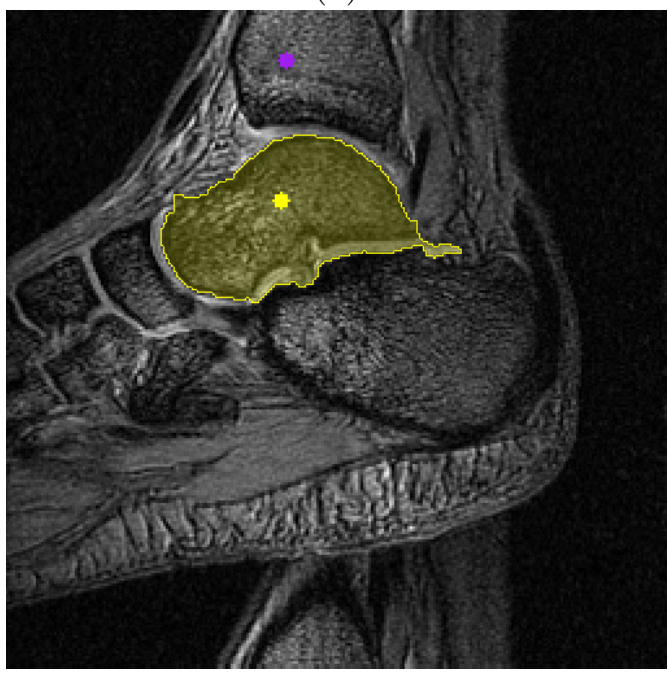

(c)



(e)

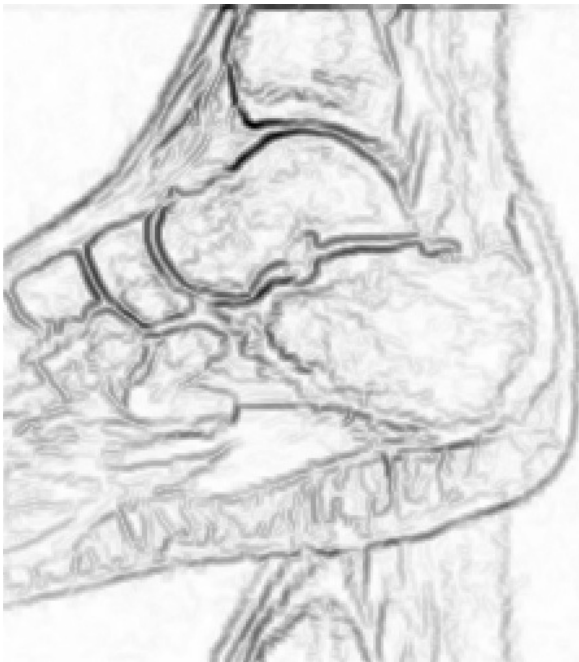

(b)

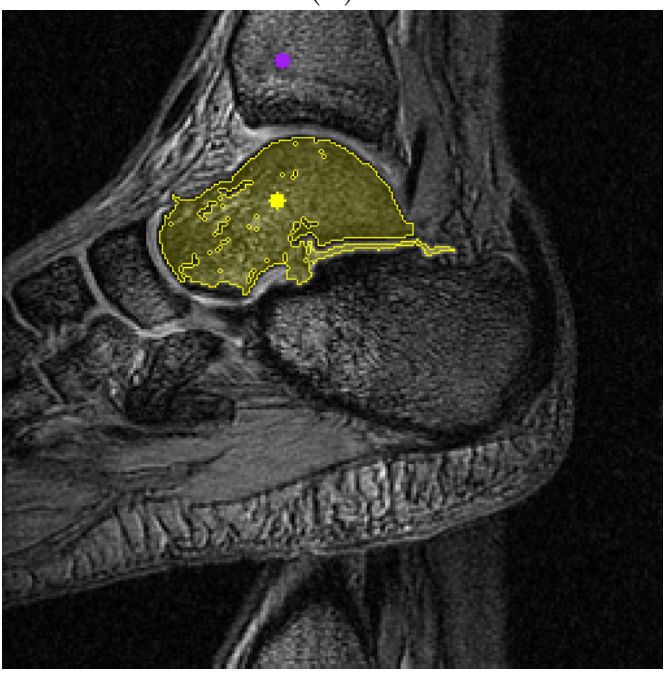

(d)

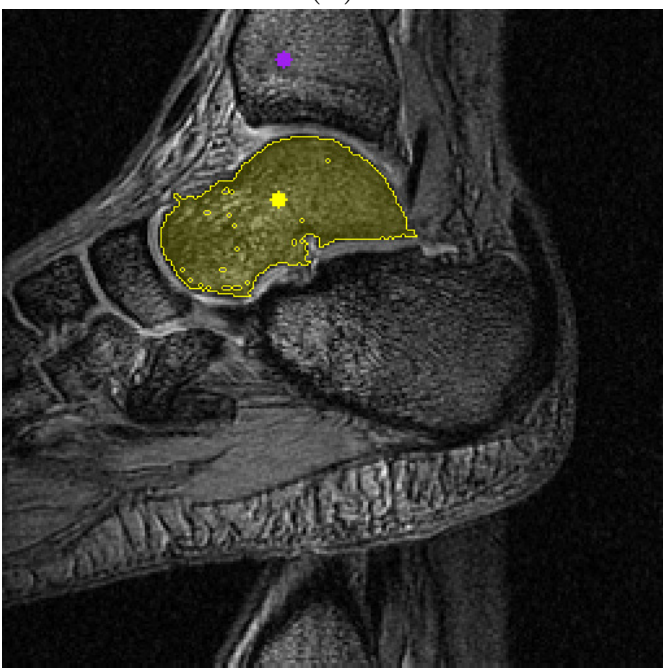

(f)

Figura 5.2: (a) Um osso tálus em uma fatia de $R M$ de um pé com os marcadores selecionados pelo usuário. (b) A atribuição de peso baseada na imagem conforme proposto em Miranda et al. (2010b). (c-f) Os resultados da segmentação para: (c) IRFC, (d) RFC, (e) OIFT e (f) ORFC. As segmentações aparecem realçadas com sobreposição em amarelo. 


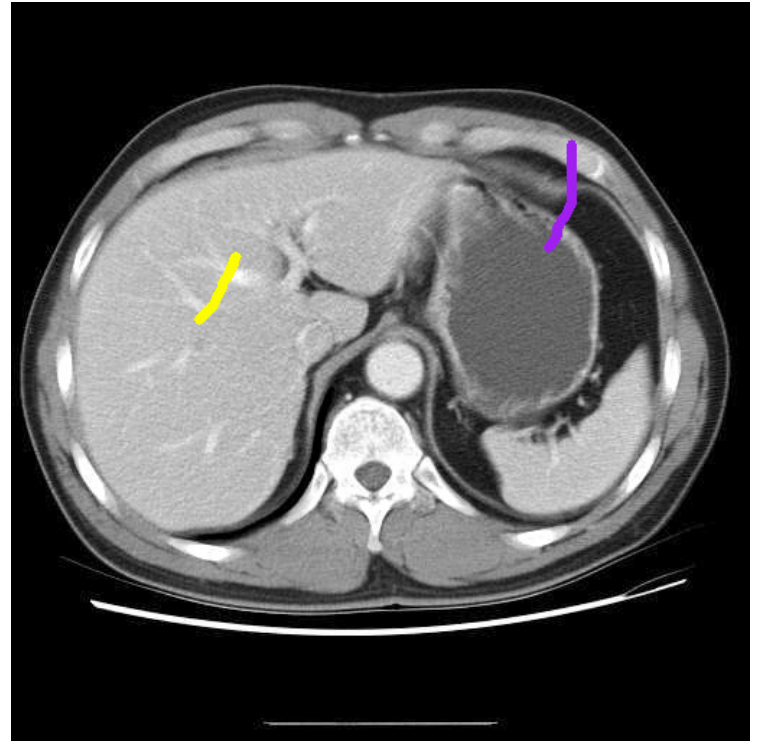

(a)

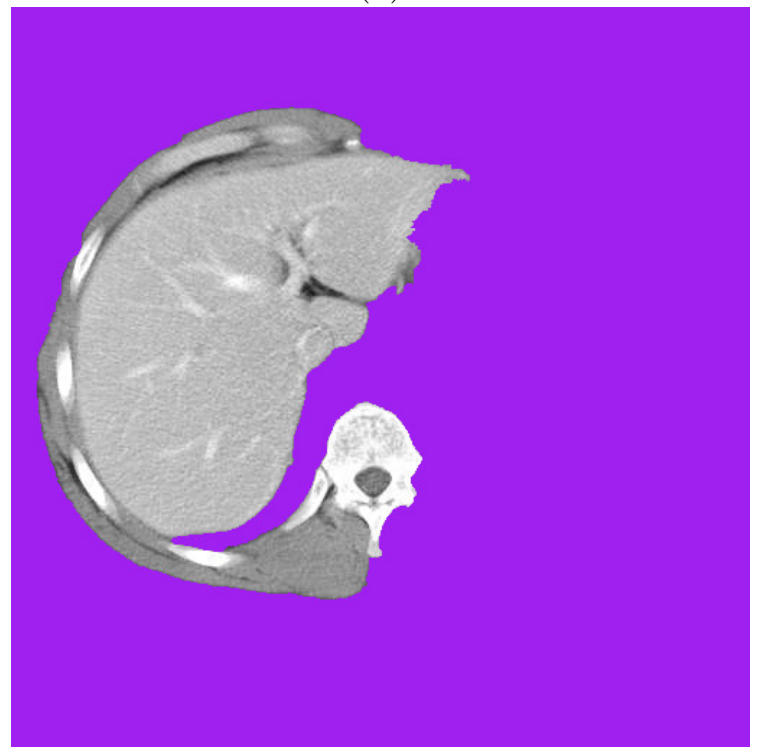

(c)

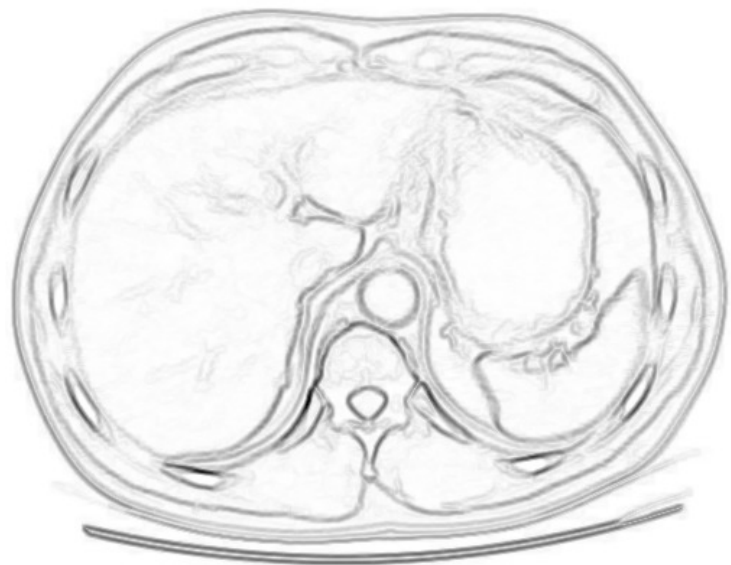

(b)



(d)

Figura 5.3: (a) Uma imagem de TC do fígado com seleção de sementes pelo usuário (b) A atribuição de peso baseada na imagem conforme proposto em Miranda et al. (2010b). (c-d) Resultado de segmentação para: (c) OIFT e (d) ORFC. 


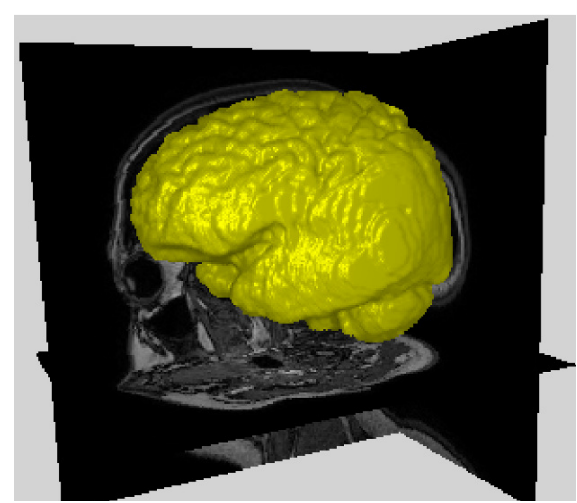

(a)

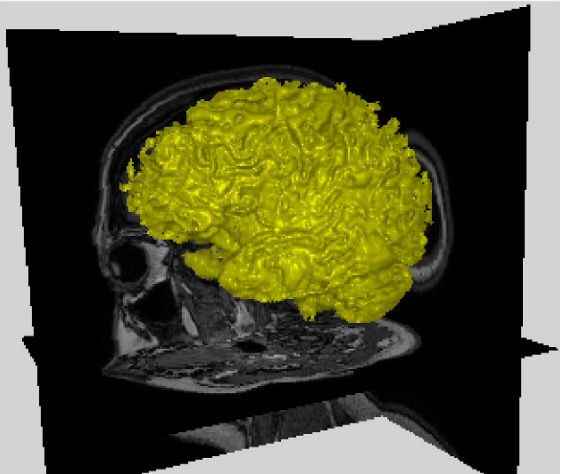

(c)

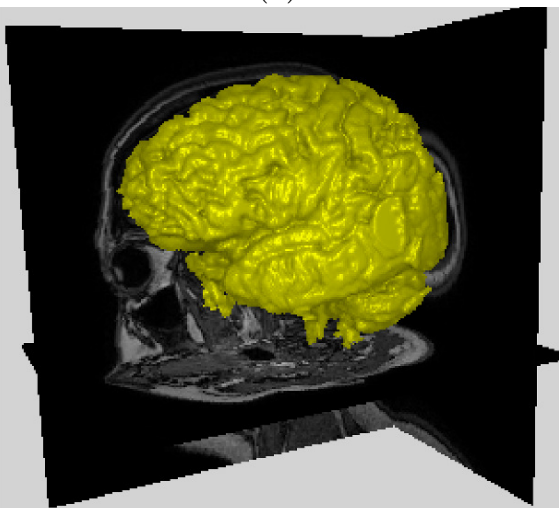

(e)

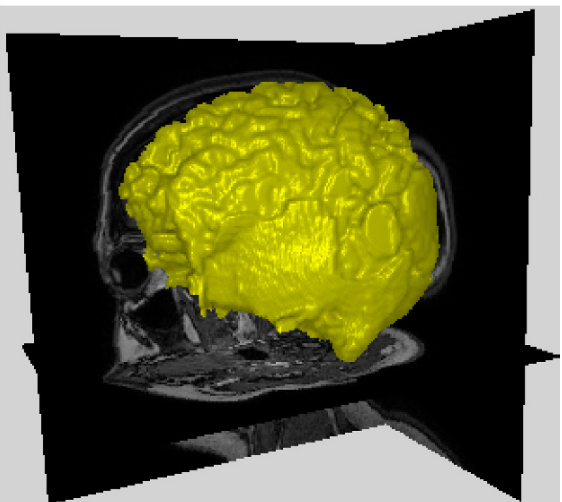

(g)

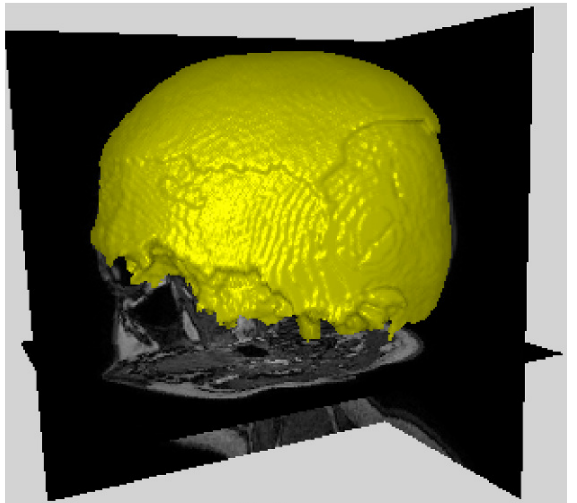

(b)

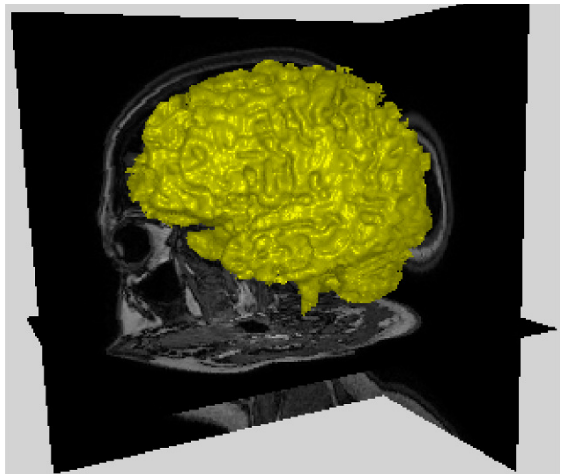

(d)

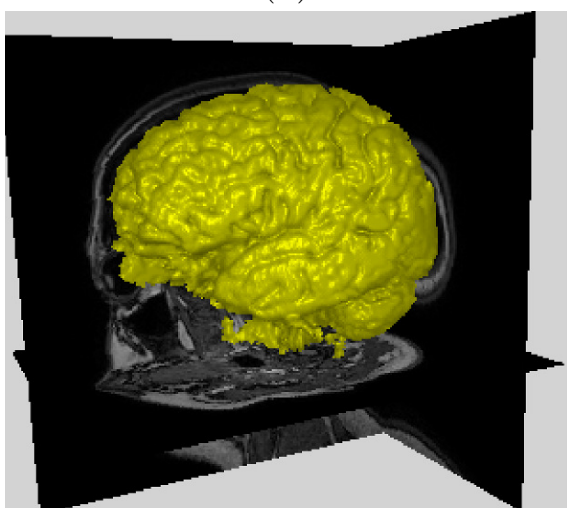

(f)

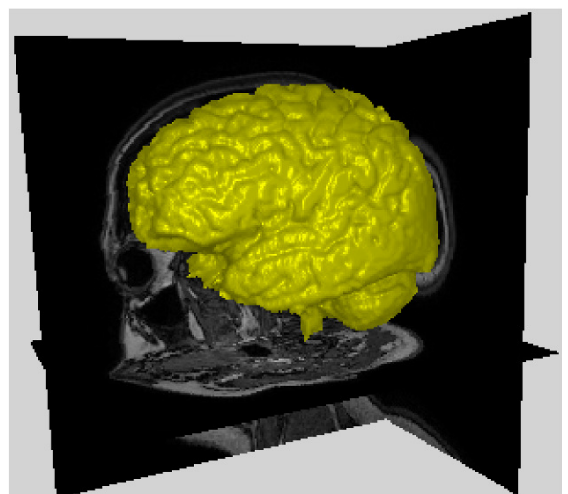

(h)

Figura 5.4: Exemplo de Skull Stripping. (a) O gabarito de segmentação. Os resultados de segmentação para: (b) GC, (c) RFC, (d) ORFC, (e) IRFC, (f) OIFT, (g) RFC+GC e (h) ORFC+GC. 


\subsection{Análise quantitativa}

Nos experimentos quantitativos foi utilizada a técnica de seleção de sementes por erosão do gabarito, onde as sementes são selecionadas por erosão do objeto e do fundo para diferentes raios de erosão, sendo que no início as sementes constituem as bordas entre o objeto e o fundo. Isto permite variar o conjunto de sementes de forma controlada e estudar a influência dos conjuntos de sementes nos resultados.

A fim de gerar uma situação mais desafiadora nos experimentos quantitativos, consideramos um raio maior de erosão para as sementes externas (duas vezes o valor do raio interno), resultando em uma disposição assimétrica das sementes (Figura 5.5).

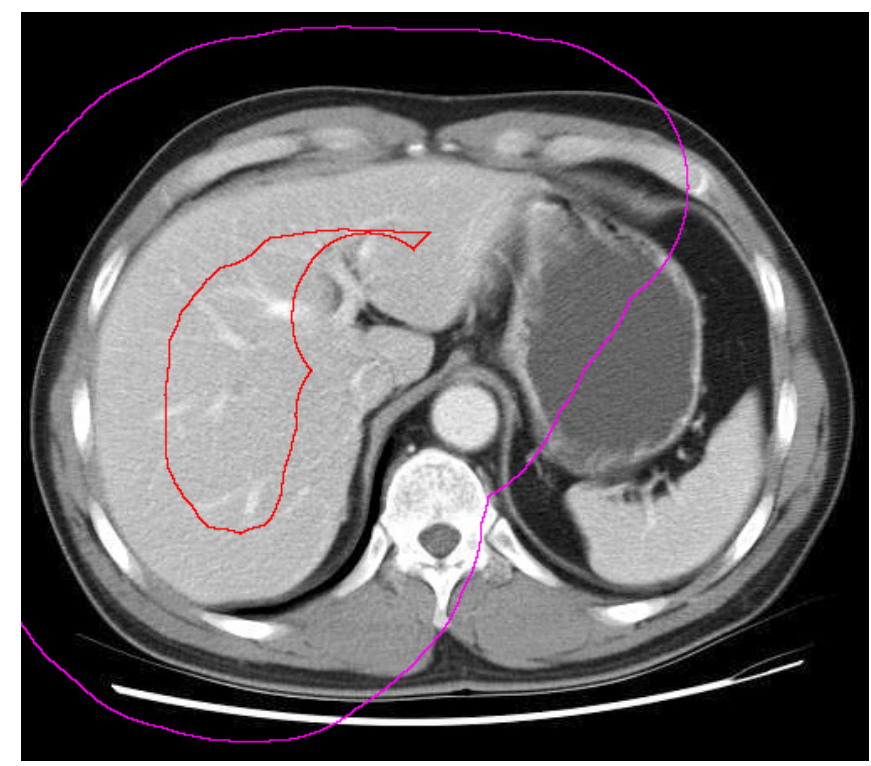

Figura 5.5: Sementes obtidas por erosão de objeto e fundo da segmentação verdadeira (gabarito).

Por uma questão de simplicidade, nos experimentos quantitativos, adotamos a atribuição de peso dada por $\delta(a, b)=K-|G(a)+G(b)|$, onde $G(a)$ denota a magnitude do gradiente do operador Sobel. Na Equação 3.8, consideramos $\alpha=0.5$ em todos os experimentos que envolvem OIFT, GC, ORFC e ORFC+GC (ORFC combinado com o método híbrido de Corte em Grafo); e $\alpha=0.0$ no caso de abordagens não dirigidas. O valor $\alpha=0.5$ é o valor padrão adotado em resultados experimentais [Mansilla e Miranda (2013a); Miranda e Mansilla (2014)], que é uma configuração bem mais equilibrada. Para valores baixos $(\alpha \approx 0)$, os métodos orientados (por exemplo, ORFC) degeneram em seus abordagens não dirigidas originais (por exemplo, RFC), e para valores elevados, os métodos orientados podem tornar-se mais sensíveis ao ruído. Usamos $A_{O R F C}^{i n, \text {, }}$ para o ossos do pé, pois apresentam transições de escuro para pixels claros; e $A_{O R F C}^{\text {out,ox }}$ para o fígado, já que esse tem a orientação oposta.

No primeiro experimento, foram utilizadas 40 fatias de imagens por ressonância magnética (RM) de ossos do pé. Foi realizada a segmentação dos ossos tálus e calcâneo para todos os métodos (IRFC [Ciesielski et al. (2007)], RFC [Saha e Udupa (2001)], OIFT [Mansilla e Miranda (2013a)], RFC + GC [Ciesielski et al. (2013)], GC - o Corte em Grafo com polaridade de borda [Boykov e Funka-Lea (2006)], ORFC [Bejar e Miranda (2014)], e o proposto método híbrido $\mathrm{ORFC}+\mathrm{GC}$ ), para diferentes conjuntos de sementes obtidos automaticamente ao erodir e dilatar os gabaritos de segmentação para diferentes valores de raio. Ao variar o valor do raio, podemos repetir a segmentação para diferentes conjuntos de sementes e traçar as curvas de acurácia, utilizando o coeficiente de similaridade de Dice, e curvas de erro de falso-positivo (normalizado pelo 
tamanho do objeto) (Figura 5.6). Na Figura 5.7 foram traçadas as curvas de tempo de execução de todos os métodos usando o conjunto de dados do calcanêo e tálus.

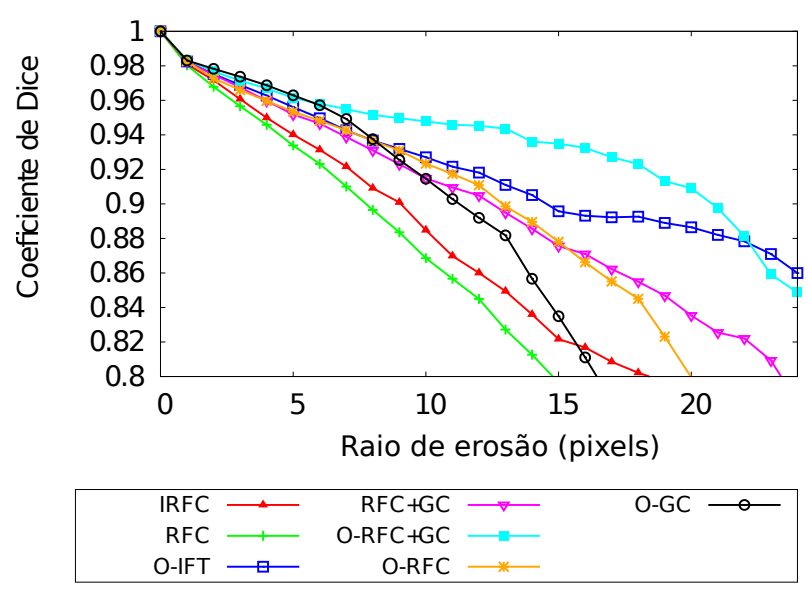

(a)

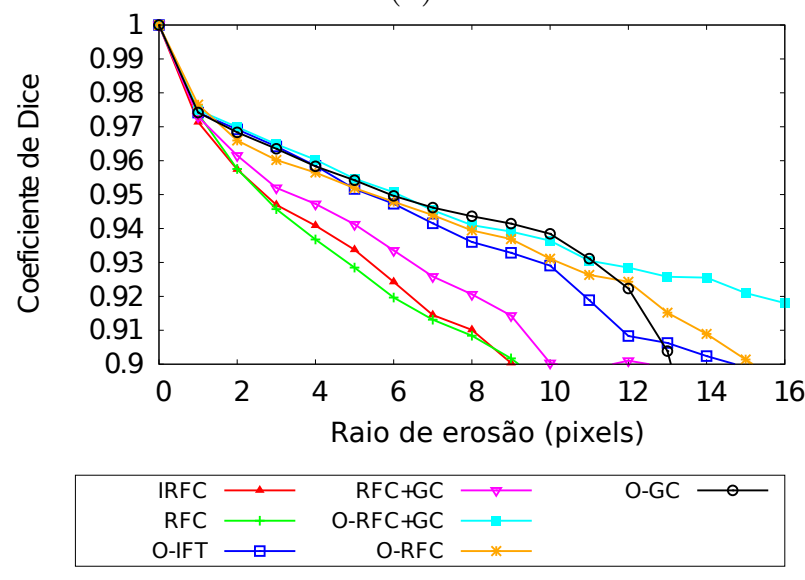

(c)

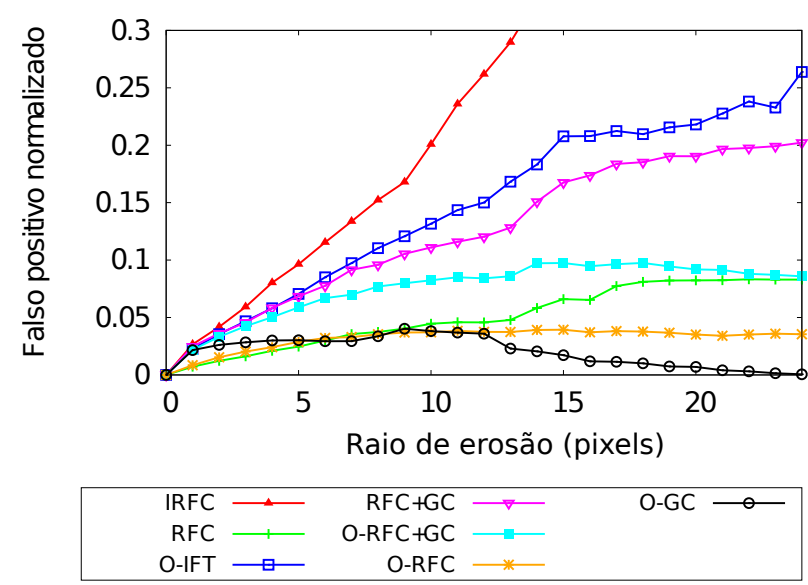

(b)

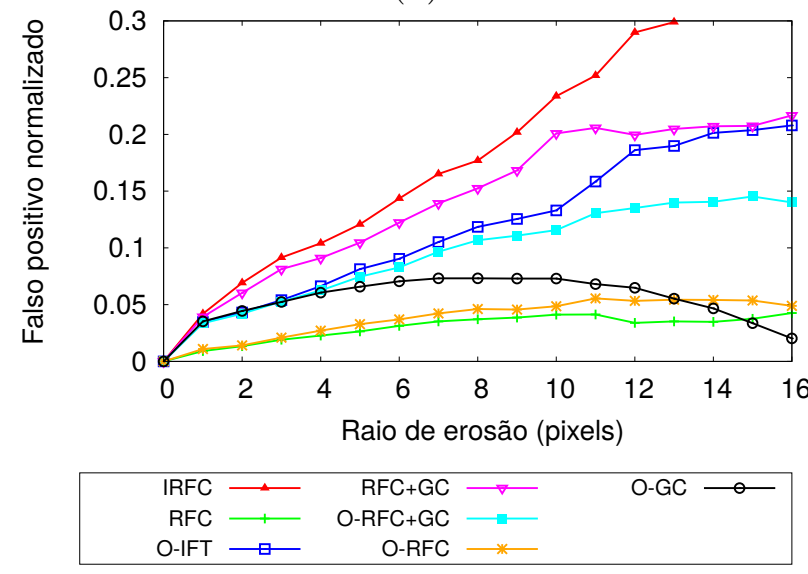

(d)

Figura 5.6: As curvas experimentais para os conjuntos de dados em 2-D. Curva média de acurácia (coeficiente de similaridade de Dice) e as curvas de falsos positivos normalizados, utilizando sementes desigualmente erodidas, para segmentar: (a-b) calcâneo e (c-d) tálus.

No segundo experimento, 40 imagens de fatias de estudos do toráx de tomografia computadorizada (TC) de 10 indivíduos foram utilizadas para segmentar o fígado, seguindo o mesmo procedimento para a seleção das sementes (Figura 5.5). As Figuras 5.8 e 5.9 mostram as curvas de acurácia e erro, e as curvas de tempo de execução para todos os métodos.

Uma vez que os objetos considerados apresentam buracos, nós utilizamos um pós-processamento por fechamento de buracos (CoH - Closing of Holes) [Falcão et al. (2001)], para RFC e ORFC. De fato, RFC é conhecido por potencialmente excluir regiões no interior do objeto rodeadas por arestas fortes. Note que o seu método de contrapartida com orientação, ORFC, também herda esta característica.

Em geral, os resultados mostram que ORFC pode alcançar valores altos de acurácia, com baixos erros de falsos positivos. Portanto, poderia ser combinado com outros métodos poderosos em abordagens híbridas. De fato, a abordagem híbrida ORFC+GC apresentou os melhores resultados para o osso calcâneo, sendo mais robusta do que o método GC (Graph Cut) com respeito à escolha de sementes. O método GC apresenta uma queda de acurácia para valores de raio mais elevados devido ao "problema de encolhimento". ORFC apresentou os melhores resultados para a segmentação do fígado, aproveitando o interior homogêneo. Para o tálus, ORFC mostrou uma precisão seme- 


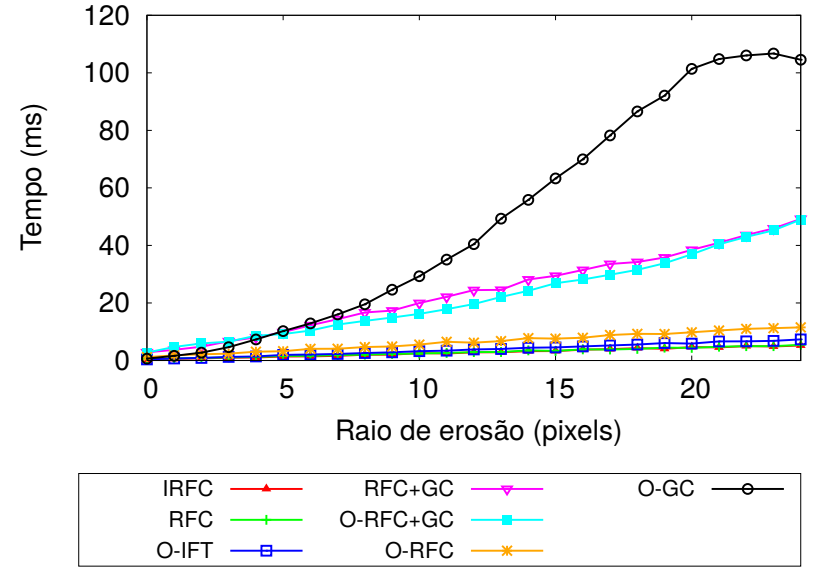

(a)

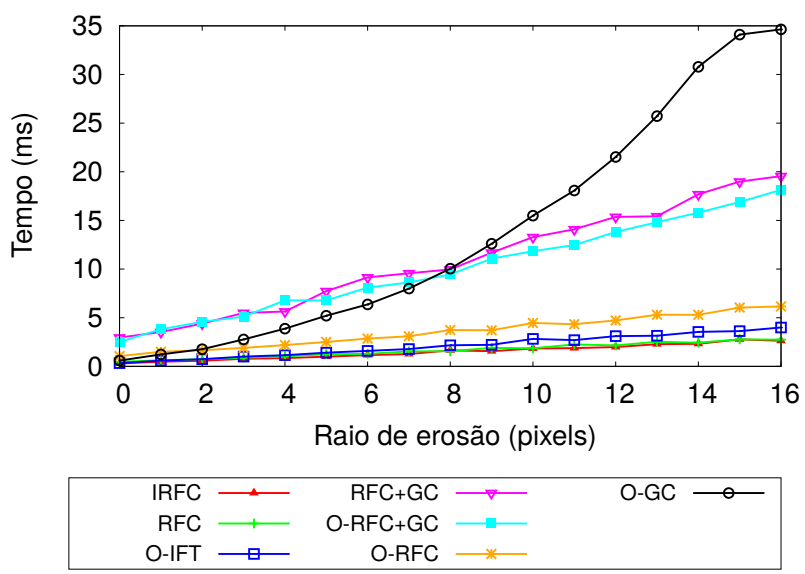

(b)

Figura 5.7: As curvas experimentais para os conjuntos de dados em 2-D. Curva de tempo de execução, utilizando sementes desigualmente erodidas, para segmentar: (a) calcâneo e (b) tálus.

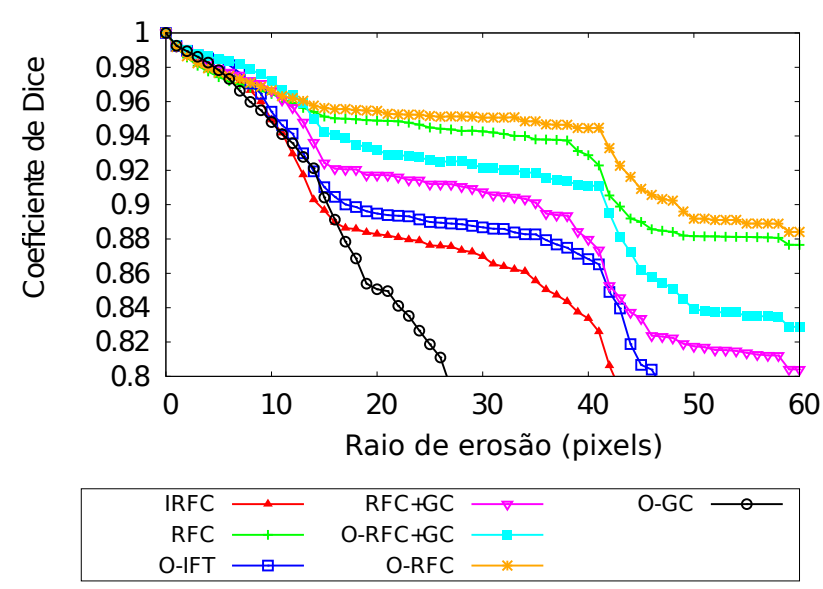

(a)

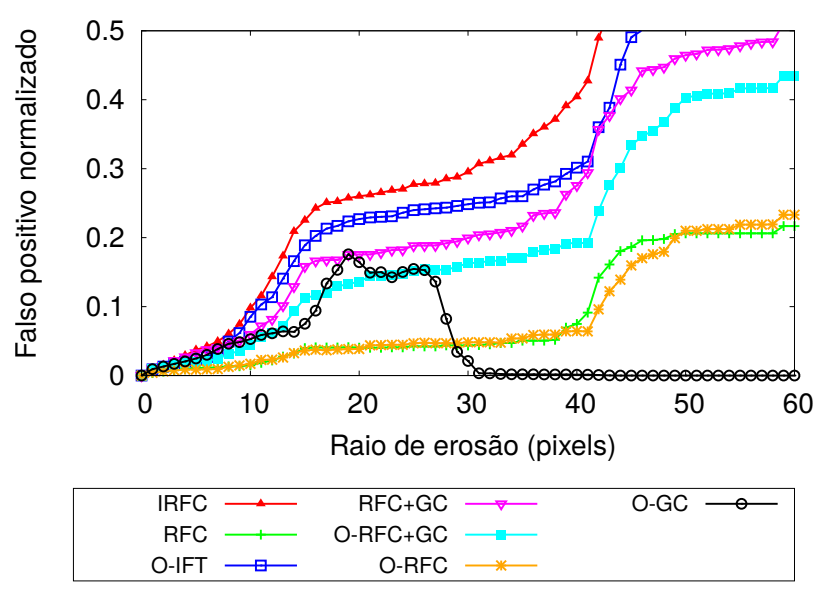

(b)

Figura 5.8: As curvas experimentais para os conjuntos de dados em 2-D. (a) Curva média de acurácia (coeficiente de similaridade de Dice) e (b) as curvas de falsos positivos normalizados, utilizando sementes desigualmente erodidas, para segmentar o fígado.

lhante do que ORFC+GC, mas com uma taxa mais baixa de falso positivo e sendo menos demorado

Também foram repetidos os experimentos usando dois conjuntos de dados tridimensionais. No primeiro caso, um conjunto de dados por ressonância magnética do cérebro humano com a sequência T1 (RM-T1) foi utilizado para segmentar o cerebelo. As imagens foram obtidas de 20 indivíduos normais de ambos gêneros, na faixa etária de 16 a 49 anos. Figura 5.10 mostra as curvas experimentais. Para o segundo conjunto de dados, foi considerado a tarefa de segmentação do cérebro (Skull Stripping) como na Figura 5.4, isto é, para eliminar fundo, ossos, olhos, pele e vasos sanguíneos, utilizando dez imagens de $\mathbf{3}$ Tesla $\mathbf{M R}-\mathbf{T} \mathbf{1}^{2}$, que incluem a cabeça e, pelo menos, uma pequena porção do pescoço de adultos do sexo masculino e do sexo feminino com cérebros normais. Os métodos RFC e ORFC têm um fraco desempenho nesses conjuntos de dados devido a ausência de um contraste nítido entre as estruturas. No entanto, a abordagem híbrida ORFC+GC apresentou os melhores resultados globais, demonstrando a importância dos métodos híbridos, e tornando

\footnotetext{
${ }^{2} 3$ Tesla MR-T1: Técnica de digitalização de imagens por ressonância magnética
} 


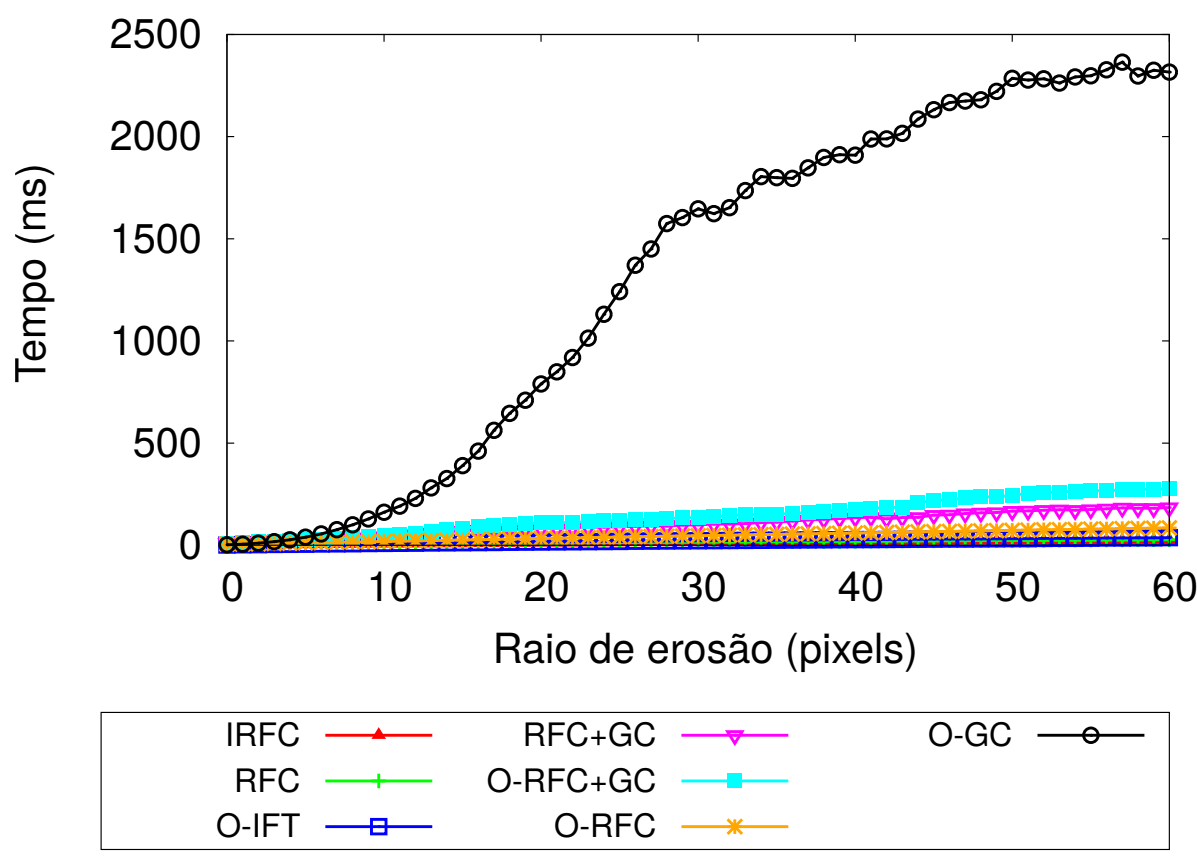

Figura 5.9: As curvas experimentais para os conjuntos de dados em 2-D. Curva de tempo de execução, utilizando sementes desigualmente erodidas, para segmentar o fígado.

evidente que, mesmo nestes casos, ORFC pode contribuir para melhorar o delineamento de corte em grafo (GC), e para reduzir seu tempo de execução. 


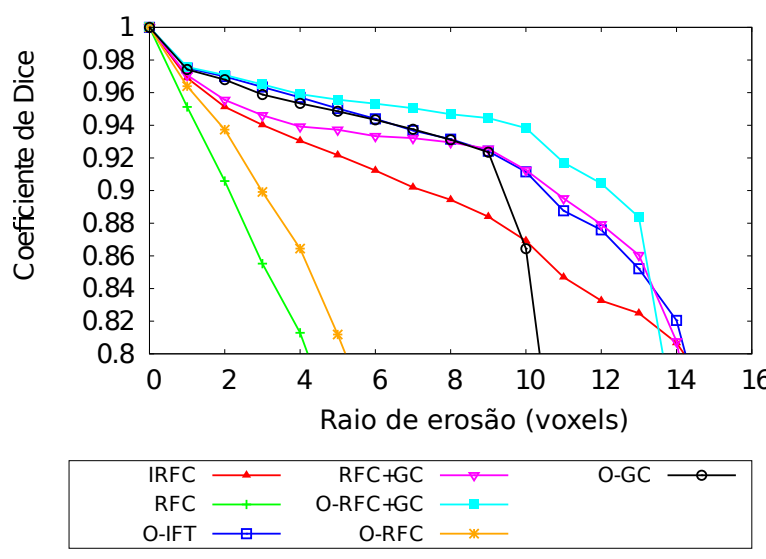

(a)
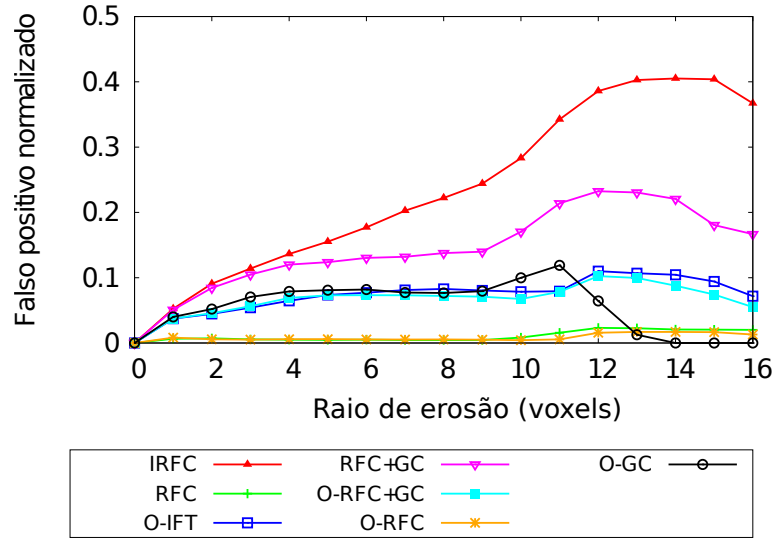

(c)

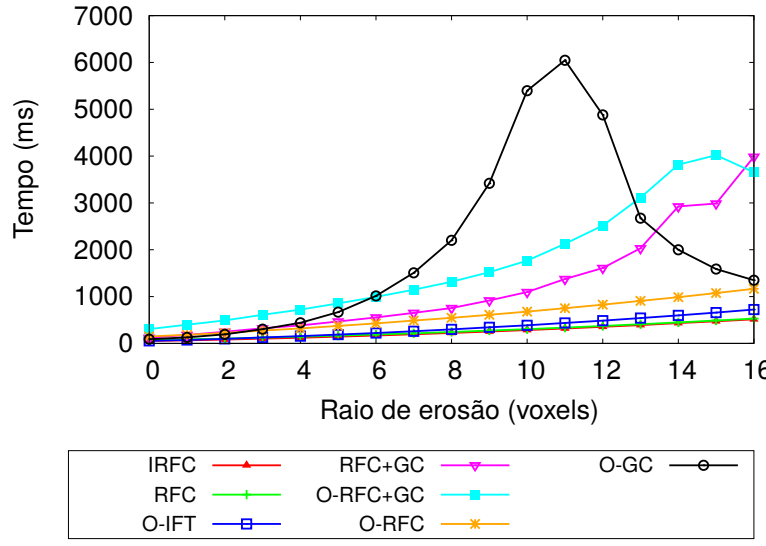

(e)
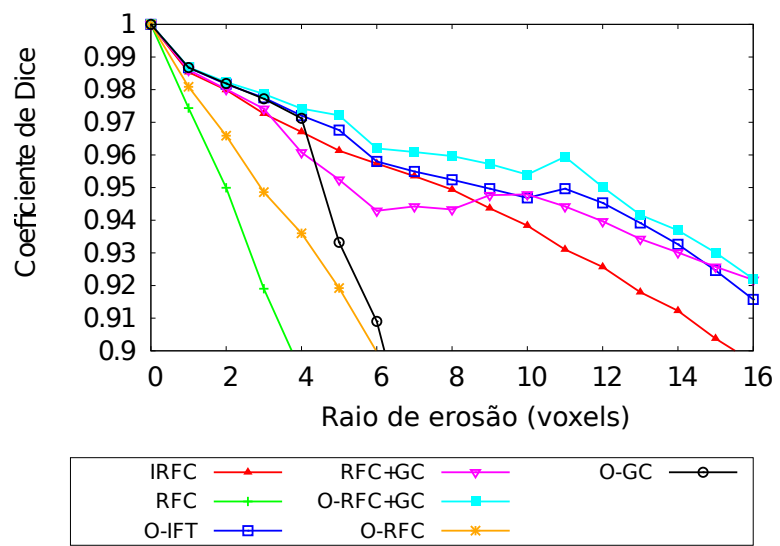

(b)
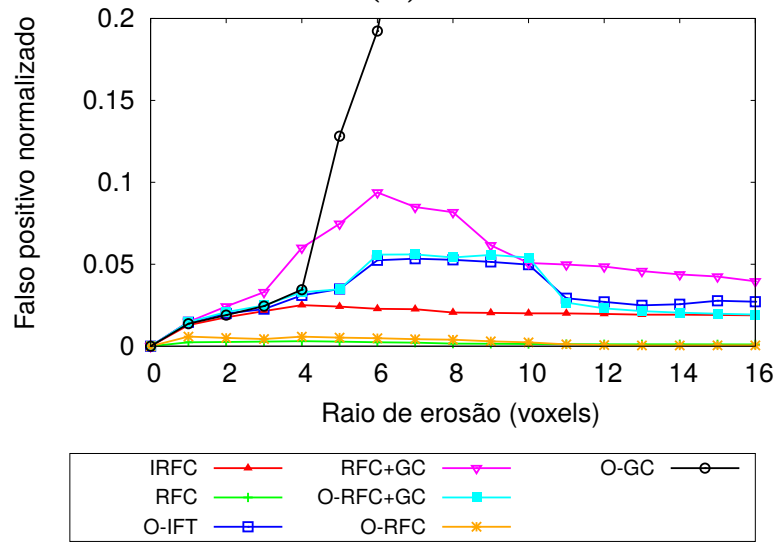

(d)

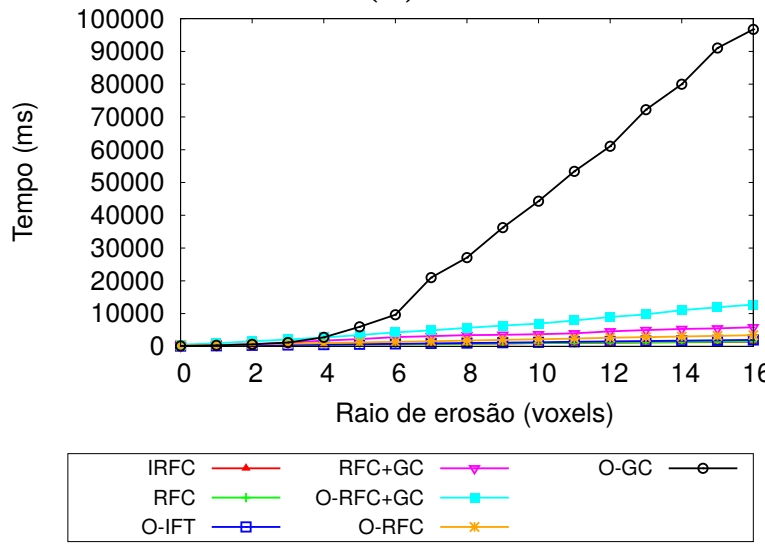

(f)

Figura 5.10: As curvas experimentais para os conjuntos de dados em 3D. Curva média de acurácia (coeficiente de similaridade de Dice) e as curvas de falsos positivos normalizados, utilizando sementes desigualmente erodidas, para segmentar: $(\mathbf{a}, \mathbf{c}, \mathbf{e})$ o cerebelo e $(\mathbf{b}, \mathbf{d}, \mathbf{f})$ o cérebro completo. 


\section{Capítulo 6}

\section{Conclusões}

Essa dissertação teve como principal contribuição a proposta de extensões para os métodos RFC [Ciesielski et al. (2012a); Saha e Udupa (2001); Udupa et al. (2002)] e RFC+GC [Ciesielski et al. (2013)], de modo a permitir a incorporação da polaridade de borda, o que possibilitou um melhor desempenho dos métodos nas bases de imagens médicas testadas, permitindo os métodos melhor se adaptarem as características específicas das transições de borda (claro para escuro ou escuro para claro) dos objetos de interesse.

Diversas técnicas foram estudadas, tanto para desenvolver o método híbrido quanto para adicionar a característica de polaridade de borda, além de métricas para comparar segmentações. O algoritmo ORFC foi parte importante para o método híbrido proposto, sendo um pré-requisito para a implementação final, enquanto que o GC naturalmente já é definido para grafos dirigidos, sendo portanto possível aproveitar implementações já existentes [Boykov e Kolmogorov (2004)]. Em virtude do falso positivo baixo da abordagem ORFC é que o método híbrido conseguiu aperfeiçoar a segmentação e superar o problema de viés de encolhimento do GC.

Destaca-se que no processo de segmentar imagens com ORFC, os buracos são um problema que pode ser resolvido através de um pós-processamento de fechamento de buracos $(\mathrm{CoH})$. No método híbrido os buracos são preenchidos naturalmente. Os algoritmos propostos são mais robustos em relação ao problema de viés de encolhimento. Em geral os experimentos realizados ao longo do desenvolvimento da abordagem híbrida mostraram resultados bastante satisfatórios, sendo recomendado para a segmentação interativa de imagens, aliás o algoritmo ORFC também alcançou valores altos de acurácia em algumas aplicações, com baixos erros de falsos positivos. Na segmentação do fígado por exemplo o método ORFC apresentou melhores resultados devido ao interior homogêneo do objeto de interesse.

A análise teórica das relações do ORFC com a OIFT só foi possível explorando a definição por corte em grafo, o que demonstra a importância do arcabouço de corte em grafo generalizado (GGC) [C.Couprie et al. (2010); Ciesielski et al. (2012b)]. No trabalho de Audigier e Lotufo (2007) foi observado que o objeto delineado via RFC corresponde ao núcleo da IFT-Watershed. Neste trabalho foi demonstrado que o mesmo não acontece com os métodos baseados em dígrafo, sendo o núcleo da OIFT maior do que o do ORFC.

\subsection{Contribuições}

As principais contribuições deste trabalho são:

- Desenvolvimento de um método de segmentação interativa, que é o algoritmo de conexidade fuzzy relativa com orientação (ORFC) [Bejar e Miranda (2014)] baseado em dígrafo que utiliza a informação de borda. 
- Desenvolvimento de um método híbrido ORFC+GC (ORFC combinado com o método de Corte em Grafo) baseado em grafos dirigidos, que possui duas versões, de acordo com a polaridade/orientação de borda, uma para o corte interno e outra para o corte externo favorecendo as transições de escuro para claro e vice-versa.

\subsection{Dificuldades}

- A segmentação de imagens médicas é uma tarefa desafiadora no processamento de imagens, por conta das bordas ambíguas do objeto de interesse, os efeitos de volume parcial, a inomogeneidade do campo magnético na aquisição da imagem, e o tamanho elevado das imagens em 3-D. O uso da polaridade de borda proposto nesse trabalho ajuda a amenizar esses problemas.

- Uma outra questão a ser considerada são os resultados insatisfatórios obtidos da definição por funções de conexidade inversa do ORFC, apresentando regiões desconexas. Esta dificuldade foi superada com o uso da definição baseada em corte.

\subsection{Sugestões para pesquisas futuras}

Como trabalho futuro, planejamos investigar o uso de restrições de convexidade geodésica em estrela (GSC - Geodesic Star Convexity) no ORFC e no método híbrido ORFC+GC (de modo similar ao que foi feito em Mansilla e Miranda (2013b)).

Outro estudo possível é combinar os métodos propostos com modelos de objeto fuzzy [Udupa et al. (2014)] para obter um resultado de segmentação automático.

Os algoritmos podem ser paralelizados de modo a rodarem ao mesmo tempo em diferentes processos, fazendo uso dos múltiplos núcleos de um mesmo processador, por exemplo.

Outra pesquisa complementar corresponderia ao estudo da região de robustez do ORFC e o desenvolvimento de uma definição formal de um algoritmo para calcular o núcleo do ORFC, para fazer uma análise empírica das suas dimensões relativas. 


\section{Referências Bibliográficas}

Audigier e Lotufo (2007) R. Audigier e R.A. Lotufo. Seed-relative segmentation robustness of watershed and fuzzy connectedness approaches. Em Proceedings of the XX Brazilian Symposium on Computer Graphics and Image Processing (SIBGRAPI), páginas 61-68, Belo Horizonte, MG. IEEE CPS. Citado na pág. 1, 34, 51

Bai e Sapiro (2007) X. Bai e G. Sapiro. Distance cut: Interactive segmentation and matting of images and videos. Em Proc. of the IEEE Intl. Conf. on Image Processing, volume 2, páginas II - 249-II - 252. Citado na pág. 1

Bejar e Miranda (2014) Hans H. C. Bejar e Paulo A.V. Miranda. Oriented relative fuzzy connectedness: Theory, algorithms, and applications in image segmentation. Em 27th SIBGRAPI Conference on Graphics, Patterns and Images, páginas 304-311. Citado na pág. 3, 4, 46, 51

Bergo e Falcão (2007) Felipe P.G. Bergo e Alexandre Falcão. A partitioned algorithm for the image foresting transform. Em Proceedings of the Eighth International Symposium on Mathematical Morphology, páginas 425-436, São José dos Campos, SP, Brazil. Gerald J. F. Banon, MCT/INPE. ISBN 978-85-17-00032-4. URL http://urlib.net/dpi.inpe.br/ismm@80/2007/04.13. 17.56. Citado na pág. 13

Boykov e Funka-Lea (2006) Y. Boykov e G. Funka-Lea. Graph cuts and efficient N-D image segmentation. Intl. Jrnl. of Comp. Vision, 70(2):109-131. ISSN 0920-5691. Citado na pág. 1, 2, 4, $19,22,38,46$

Boykov e Kolmogorov (2004) Y. Boykov e V. Kolmogorov. An experimental comparison of min-cut/max-flow algorithms for energy minimization in vision segmentation with connectivity priors. Em IEEE Transactions on Pattern Analysis and Machine Intelligence, páginas 1124-1137. IEEE. Citado na pág. 2, 38, 51

Camilus e Govindan (2012) K. S. Camilus e V. K. Govindan. A review on graph based segmentation. Intl. Journal of Image, Graphics and Signal Processing, 4(5):1-13. ISSN 2074-9074. Citado na pág. 1

Cappabianco et al. (2007) F.A.M. Cappabianco, G. Araujo e A.X. Falcao. The image forest transform architecture. Em Field-Programmable Technology, 200\%. ICFPT 200\%. International Conference on, páginas 137-144. doi: 10.1109/FPT.2007.4439242. Citado na pág. 13

Ccacyahuillca Bejar e Miranda (2015) Hans Harley Ccacyahuillca Bejar e Paulo A V Miranda. Oriented relative fuzzy connectedness: theory, algorithms, and its applications in hybrid image segmentation methods. EURASIP Journal on Image and Video Processing, 2015(1):21. doi: 10.1186/s13640-015-0067-4. URL http://dx.doi.org/10.1186/s13640-015-0067-4. Citado na pág. 3

C.Couprie et al. (2010) C.Couprie, L.Grady, L.Najman e H.Talbot. Power watersheds: A unifying graph-based optimization framework. IEEE Transactions on Pattern Analysis and Machine Intelligence, 99. ISSN 0162-8828. doi: http://doi.ieeecomputersociety.org/10.1109/TPAMI.2010.200. Citado na pág. 1, 51 
Ciesielski e Udupa (2011) K.C. Ciesielski e J.K. Udupa. Chapter: Region-based segmentation: fuzzy connectedness, graph cut, and other related algorithms in: Biomedical Image Processing. Springer-Verlag. (T.M. Deserno, ed.). Citado na pág. 3, 17

Ciesielski e Udupa (2010a) K.C. Ciesielski e J.K. Udupa. Affinity functions in fuzzy connectedness based image segmentation i: Equivalence of affinities. Computer Vision and Image Understanding, 114(1):146-154. ISSN 1077-3142. Citado na pág. 18

Ciesielski e Udupa (2010b) K.C. Ciesielski e J.K. Udupa. Affinity functions in fuzzy connectedness based image segmentation ii: Defining and recognizing truly novel affinities. Computer Vision and Image Understanding, 114(1):155-166. ISSN 1077-3142. Citado na pág. 18

Ciesielski et al. (2007) K.C. Ciesielski, J.K. Udupa, P.K. Saha e Y. Zhuge. Iterative relative fuzzy connectedness for multiple objects with multiple seeds. Computer Vision and Image Understanding, 107(3):160-182. ISSN 1077-3142. Citado na pág. xv, 1, 18, 42, 46

Ciesielski et al. (2012a) K.C. Ciesielski, J.K. Udupa, A.X. Falcão e P.A.V. Miranda. Fuzzy connectedness image segmentation in graph cut formulation: A linear-time algorithm and a comparative analysis. Journal of Mathematical Imaging and Vision, 44(3):375-398. Citado na pág. 1, 3, $14,17,38,51$

Ciesielski et al. (2012b) K.C. Ciesielski, J.K. Udupa, A.X. Falcão e P.A.V. Miranda. A unifying graph-cut image segmentation framework: algorithms it encompasses and equivalences among them. Em Proc. of SPIE on Medical Imaging: Image Processing, volume 8314. doi: 10.1117/12. 911810. Citado na pág. 1, 51

Ciesielski et al. (2013) Krzysztof Chris Ciesielski, P.A.V. Miranda, A.X. Falcão e Jayaram K. Udupa. Joint graph cut and relative fuzzy connectedness image segmentation algorithm. Medical Image Analysis (MEDIA), 17(8):1046-1057. Citado na pág. xv, 2, 3, 18, 22, 37, 38, 41, 42, 46, 51

Comas e Meschino (2014) Diego S. Comas e Gustavo J.Y. Meschino. Segmentación de imágenes mediante reconocimiento de patrones. Em Escuela y Workshop Argentino en Ciencias de las Imágenes, páginas 1-48, Buenos Aires, AR. ECImag. Citado na pág. 8, 9

Cousty et al. (2010) J. Cousty, G. Bertrand, L. Najman e M. Couprie. Watershed cuts: Thinnings, shortest path forests, and topological watersheds. Trans. on Pattern Analysis and Machine Intelligence, 32:925-939. ISSN 0162-8828. doi: http://doi.ieeecomputersociety.org/10.1109/TPAMI. 2009.71. Citado na pág. 1

Dice (1945) Lee R. Dice. Measures of the Amount of Ecologic Association Between Species, volume 26. Ecological Society of America, Washington, DC, USA. doi: http://www.jstor.org/ stable/1932409. Citado na pág. 9

Falcão et al. (1998) Alexandre X. Falcão, Jayaram K. Udupa, Supun Samarasekera, Shoba Sharma, Bruce Elliot Hirsch e Roberto de A. Lotufo. User-steered image segmentation paradigms: Live wire and live lane. Graphical Models and Image Processing, 60(4):233 - 260. ISSN 1077-3169. doi: http://dx.doi.org/10.1006/gmip.1998.0475. URL http://www.sciencedirect.com/science/article/ pii/S1077316998904750. Citado na pág. 2, 13

Falcão e Bergo (2004) A.X. Falcão e F.P.G. Bergo. Interactive volume segmentation with differential image foresting transforms. Medical Imaging, IEEE Transactions on, 23(9):1100-1108. ISSN 0278-0062. doi: 10.1109/TMI.2004.829335. Citado na pág. 13

Falcão et al. (2001) A.X. Falcão, B.S. da Cunha e R.A. Lotufo. Design of connected operators using the image foresting transform. Em Proceedings of SPIE on Medical Imaging, volume 4322, páginas 468-479. Citado na pág. 13, 47 
Falcão et al. (2004) A.X. Falcão, J. Stolfi e R.A. Lotufo. The image foresting transform: Theory, algorithms, and applications. IEEE Transactions on Pattern Analysis and Machine Intelligence, 26(1):19-29. Citado na pág. 1, 4, 13, 17, 19

Grady (2006) L. Grady. Random walks for image segmentation. IEEE Trans. Pattern Anaysis and Machine Intelligence, 28(11):1768-1783. ISSN 0162-8828. doi: http://dx.doi.org/10.1109/ TPAMI.2006.233. Citado na pág. 1

Gulshan et al. (2010) V. Gulshan, C. Rother, A. Criminisi, A. Blake e A. Zisserman. Geodesic star convexity for interactive image segmentation. Em IEEE Conference on Computer Vision and Pattern Recognition. IEEE. Citado na pág. 1

J.P. Papa e Miranda (2007) A. Falcão J.P. Papa e P.A.V. Miranda. Design of robust pattern classifiers based on optimum-path forests. Em Proceedings of the Eighth International Symposium on Mathematical Morphology, páginas 337-348, São José dos Campos, SP, Brazil. Gerald J. F. Banon, MCT/INPE. ISBN 978-85-17-00032-4. URL http://urlib.net/dpi.inpe.br/ismm@80/ 2007/04.13.23.19. Citado na pág. 14

Kalpathy-Cramer e Müller (2011) J. Kalpathy-Cramer e H. Müller. Chapter: Systematic Evaluations and Ground Truth: Biomedical Image Processing. Springer-Verlag. (T.M. Deserno, ed.). Citado na pág. 9

Kaufmann (1975) A. Kaufmann. Introduction to the Theory of Fuzzy Subsets, volume 1. Academic Press. ISBN 0124023010. URL http://www.amazon.com/exec/obidos/redirect?tag= citeulike07-20\&path=ASIN/0124023010. Citado na pág. 31

Lézoray e Grady (2012) O. Lézoray e L. Grady. Image Processing and Analysis with Graphs: Theory and Practice. CRC Press, California, USA. ISBN 1439855072. Citado na pág. xiii, 1, 2, 3

Lotufo et al. (2002) R.A. Lotufo, A.X. Falcão e F. Zampirolli. IFT-Watershed from gray-scale marker. Em Proceedings of the XV Brazilian Symposium on Computer Graphics and Image Processing, páginas 146-152. IEEE. Citado na pág. 1

Mansilla e Miranda (2013a) L.A.C. Mansilla e P.A.V. Miranda. Image segmentation by oriented image foresting transform: Handling ties and colored images. Em 18th Intl. Conf. on Digital Signal Processing, páginas 1-6, Greece. Citado na pág. 19, 23, 31, 46

Mansilla e Miranda (2013b) LucyA.C. Mansilla e PauloA.V. Miranda. Image segmentation by oriented image foresting transform with geodesic star convexity. Em Richard Wilson, Edwin Hancock, Adrian Bors e William Smith, editors, Computer Analysis of Images and Patterns, volume 8047 of Lecture Notes in Computer Science, páginas 572-579. Springer Berlin Heidelberg. ISBN 978-3-642-40260-9. doi: 10.1007/978-3-642-40261-6_69. URL http://dx.doi.org/10.1007/ 978-3-642-40261-6_69. Citado na pág. 52

Meyer (2002) AndrÃlia D. Meyer. Compara $\tilde{g} \breve{a} a$ de Coeficiente de Similaridade Usados em An Ãalises de Agrupamento com Dados de Marcadores Moleculares Dominantes. Biblioteca Digitais de Teses e DissertaÃğ Ãţes da USP, SÃ̌co Paulo, BR. Citado na pág. 9

Miranda et al. (2012) Paulo A. V. Miranda, Alexandre X. Falcãao e Thiago Vallin Spina. Riverbed: A novel user-steered image segmentation method based on optimum boundary tracking. IEEE Transactions on Image Processing, 21(6):3042-3052. doi: http://dx.doi.org/10.1109/TIP. 2012.2188034. Citado na pág. 2, 14

Miranda e Falcão (2009) P.A.V. Miranda e A.X. Falcão. Links between image segmentation based on optimum-path forest and minimum cut in graph. Journal of Mathematical Imaging and Vision, 35(2):128-142. ISSN 1573-7683. Citado na pág. 1 
Miranda e Falcão (2011) P.A.V. Miranda e A.X. Falcão. Elucidating the relations among seeded image segmentation methods and their possible extensions. Em XXIV Conference on Graphics, Patterns and Images, páginas 289-296, Maceió, AL. Citado na pág. 1, 14

Miranda e Mansilla (2014) P.A.V. Miranda e L.A.C. Mansilla. Oriented image foresting transform segmentation by seed competition. IEEE Transactions on Image Processing, 23(1):389-398. Citado na pág. xv, 2, 4, 19, 23, 31, 42, 46

Miranda et al. (2010a) P.A.V. Miranda, A.X. Falcão e G. Ruppert. How to complete any segmentation process interactively via image foresting transform. Em 23rd SIBGRAPI: Conf. on Graphics, Patterns and Images, páginas 309-316. Citado na pág. 34

Miranda et al. (2010b) P.A.V. Miranda, A.X. Falcão e J.K. Udupa. Synergistic arc-weight estimation for interactive image segmentation using graphs. Computer Vision and Image Understanding, 114(1):85-99. ISSN 1077-3142. Citado na pág. xv, xvi, 41, 43, 44

Mortensen e Barrett (1998) Eric N. Mortensen e William A. Barrett. Interactive segmentation with intelligent scissors. Graphical Models and Image Processing, 60(5):349 - 384. ISSN 1077-3169. doi: http://dx.doi.org/10.1006/gmip.1998.0480. URL http://www.sciencedirect.com/ science/article/pii/S1077316998904804. Citado na pág. 2

Price et al. (2010) Brian L. Price, Bryan Morse e Scott Cohen. Geodesic graph cut for interactive image segmentation. Em IEEE Conference on Computer Vision and Pattern Recognition, páginas 3161-3168, San Francisco, CA. IEEE. Citado na pág. 2

Rocha et al. (2009) Leonardo Marques Rocha, Fábio A. M. Cappabianco e Alexandre Xavier Falcão. Data clustering as an optimum-path forest problem with applications in image analysis. Int. J. Imaging Syst. Technol., 19(2):50-68. ISSN 0899-9457. doi: 10.1002/ima.v19:2. URL http://dx.doi.org/10.1002/ima.v19:2. Citado na pág. 14

Saha e Udupa (2001) P.K. Saha e J.K. Udupa. Relative fuzzy connectedness among multiple objects: Theory, algorithms, and applications in image segmentation. Comp. Vision and Image Understanding, 82(1):42-56. ISSN 1077-3142. Citado na pág. 1, 2, 3, 16, 31, 46, 51

Singaraju et al. (2008) D. Singaraju, L. Grady e R. Vidal. Interactive image segmentation via minimization of quadratic energies on directed graphs. Em Intl. Conf. on Computer Vision and Pattern Recognition, páginas 1-8. Citado na pág. 2

Sinop e L.Grady (2007) A.K. Sinop e L.Grady. A seeded image segmentation framework unifying graph cuts and random walker which yields a new algorithm. Em Proc. of the 11th Intl. Conf. on Computer Vision, páginas 1-8. Citado na pág. 1

Sorensen (1948) Thorvald J. Sorensen. A method of establishing groups of equal amplitude in plant sociology based on similarity of species content and its application to analyses of the vegetation on Danish commons, volume 5. I kommission hos E. Munksgaard, Washington, DC, USA. doi: http://books.google.co.in/books?id=rpS8GAAACAAJ. Citado na pág. 9

T.H. Cormen e Stein (2009) R.L. Rivest T.H. Cormen, C.E. Leiserson e C. Stein. Introduction to Algorithms. The MIT Press, London, England. ISBN 978-0-262-03384-8. Citado na pág. 21

Udupa et al. (2014) Jayaram K. Udupa, Krzysztof C. Ciesielski e Alexandre X. Falcão. Body wide hierarchical fuzzy modeling, recognition, and delineation of anatomy in medical images. volume 18, páginas 752-771. Elsevier Science Inc. Citado na pág. 1, 52

Udupa et al. (2002) J.K. Udupa, P.K. Saha e R.A. Lotufo. Relative fuzzy connectedness and object definition: Theory, algorithms, and applications in image segmentation. IEEE Transactions on Pattern Analysis and Machine Intelligence, 24:1485-1500. Citado na pág. 3, 4, 16, 51 
Vallin Spina et al. (2014) T. Vallin Spina, P.A.V. de Miranda e A. Xavier Falcao. Hybrid approaches for interactive image segmentation using the live markers paradigm. Em IEEE Conference on Computer Vision and Pattern Recognition, páginas 5756-5769, Campinas, Brazil. IEEE. Citado na pág. 1

Vezhnevets e Konouchine (2005) V. Vezhnevets e V. Konouchine. "growcut- interactive multilabel N-D image segmentation by cellular automata. Em Proc. Graphicon., páginas 150-156. Citado na pág. 1,14

Vicente et al. (2008) S. Vicente, V. Kolmogorov e C. Rother. Graph cut based image segmentation with connectivity priors. Em IEEE Conference on Computer Vision and Pattern Recognition, páginas 1-8, Anchorage, AK. IEEE. Citado na pág. 2, 22 


\section{Índice Remissivo}

algoritmo IFT, 15

avaliação

matriz confusão, 8

caminho, 7

componente conexo dirigido, 25

conexidade fuzzy relativa, 15-17

conexidade fuzzy relativa orientação, 23-39

algoritmo

externo, 25

interno, 25

corte externo, 24

corte interno, 24

núcleo, 30

conexidade inversa, 23

conexidade anti-paralelas, 23

corte em grafo

min-cut/max-flow, 19

corte mínimo, 21

viés encolhimento, 21

corte em grafo generalizado, 1

Dice, 9

dígrafo

arcos, 6

vértices, 6

experimentais, $41-49$

qualitativa, 41

quantitativa, 46

grafos, 6

imagem como grafo

adjacência, 7

imagem digital, 5

método híbrido, 37-39

algoritmo, 38

métodos híbridos, 1-2

polaridade de borda, 2

segmentação, 1, 13-22

função característica, 8 transformada imagem-floresta, 13

caminho ótimo, 14

conexidade, 14

floresta espalhamento, 14

transformada imagem-floresta orientação fator orientação, 18 